\title{
BepiColombo - Mission Overview and Science Goals
}

\author{
J. Benkhoff ${ }^{1}$ (D) G. Murakami ${ }^{2} \cdot$ W. Baumjohann ${ }^{3} \cdot$ S. Besse ${ }^{4} \cdot$ E. Bunce $^{5} \cdot$ M. Casale $^{4}$. \\ G. Cremosese ${ }^{6} \cdot$ K.-H. Glassmeier ${ }^{7} \cdot$ H. Hayakawa ${ }^{8} \cdot$ D. Heyner ${ }^{7} \cdot$ H. Hiesinger ${ }^{9}$. \\ J. Huovelin ${ }^{10} \cdot$ H. Hussmann ${ }^{11} \cdot$ V. lafolla ${ }^{12} \cdot{\text { L. } \text { less }^{13} \cdot \text { Y. Kasaba }}^{14} \cdot$ M. Kobayashi ${ }^{15}$. \\ A. Milillo ${ }^{12} \cdot$ I.G. Mitrofanov ${ }^{16} \cdot$ E. Montagnon ${ }^{17} \cdot$ M. Novara $^{1} \cdot$ S. Orsini ${ }^{12}$. \\ E. Quemerais ${ }^{18}$. U. Reininghaus ${ }^{1}$. Y. Saito ${ }^{8}$. F. Santoli ${ }^{12}$ - D. Stramaccioni ${ }^{1}$. \\ O. Sutherland ${ }^{1} \cdot$ N. Thomas ${ }^{19} \cdot$ I. Yoshikawa ${ }^{20} \cdot$ J. Zender ${ }^{1}$
}

Received: 23 September 2020 / Accepted: 11 November 2021 / Published online: 8 December 2021 (c) The Author(s) 2021

\begin{abstract}
BepiColombo is a joint mission between the European Space Agency, ESA, and the Japanese Aerospace Exploration Agency, JAXA, to perform a comprehensive exploration of Mercury. Launched on $20^{\text {th }}$ October 2018 from the European spaceport in Kourou, French Guiana, the spacecraft is now en route to Mercury.

Two orbiters have been sent to Mercury and will be put into dedicated, polar orbits around the planet to study the planet and its environment. One orbiter, Mio, is provided by JAXA, and one orbiter, MPO, is provided by ESA. The scientific payload of both spacecraft will provide detailed information necessary to understand the origin and evolution of the planet itself and its surrounding environment. Mercury is the planet closest to the Sun, the only terrestrial planet besides Earth with a self-sustained magnetic field, and the smallest planet in our Solar System. It is a key planet for understanding the evolutionary history of our Solar System and therefore also for the question of how the Earth and our Planetary System were formed.

The scientific objectives focus on a global characterization of Mercury through the investigation of its interior, surface, exosphere, and magnetosphere. In addition, instrumentation onboard BepiColombo will be used to test Einstein's theory of general relativity. Major effort was put into optimizing the scientific return of the mission by defining a payload such that individual measurements can be interrelated and complement each other.
\end{abstract}

Keywords Mercury exploration · BepiColombo · Planetary and Magnetospheric Science · Scientific Space Mission · Fundamental Physics · Surface and Interior

\section{Introduction}

Mercury is the smallest planet of our Solar System. Its diameter is only about $4880 \mathrm{~km}, 2 / 5$ of that of the Earth. Even two moons of Jupiter and Saturn, Ganymede and Titan, exceed

The BepiColombo mission to Mercury

Edited by Johannes Benkhoff, Go Murakami and Ayako Matsuoka

Extended author information available on the last page of the article 
Mercury in size. Mercury is the planet closest to the Sun and, because of its fast velocity around the Sun, it is named after the roman god of among others messages, trade and merchants. The main attribute of the Roman god Mercury is to be the 'gods' messenger' for which he has winged feet, which makes him fast. Because Mercury's orbit around the Sun lies within Earth orbit it only appears in Earth's sky in the morning or the evening.

Although Mercury can appear as a very bright object when viewed from Earth, its proximity to the Sun makes it difficult to observe from Earth. Already in the $17^{\text {th }}$ century it was noted that Mercury's orbit slightly deviates from what is to be expected from classical Newtonian mechanics. Einstein's theory of general relativity has to be considered because of the tremendous forces of gravity reigning in Mercury's quarters close to the Sun causing a perihelion shift of about 43 arc-seconds per century (Einstein 1916).

Mercury is in a $3: 2 \mathrm{spin} /$ orbit resonance as a result of tidal forces of the Sun acting on Mercury (Colombo and Shapiro 1966; Correia and Laskar 2004). Due to the resonance Mercury orbits the Sun twice while it rotates around its own rotational axis exactly three times. Mercury's axis of rotation is oriented nearly perpendicular to the planet's orbit, so that in the polar regions sunlight strikes the surface at a constant grazing angle. As a consequence, the interiors of large craters at the poles are permanently shadowed and remain perpetually cold, below 65 K. Radar images of the polar regions, first obtained in 1991 (Harmon and Slade 1992; Harmon et al. 1994), show that the large crater interiors are highly reflective at radar wavelengths possibly indicating the presence of water ice, sulfur, or supercooled silicates (Chabot et al. 2018). Mercury's highly elliptical orbit ranges between 0.308 and 0.467 astronomical units or about 46,0 million $\mathrm{km}$ and 69,8 million $\mathrm{km}$, respectively. The mean distance from the Sun is 57,9 million $\mathrm{km}$. Because of its close distance to the Sun (3 times closer than Earth) Mercury's surface experiences temperatures ranging from about $100 \mathrm{~K}$ on the night side of the planet up to about $700 \mathrm{~K}$ during the day at some equatorial regions.

Understanding Mercury is crucial to develop a better understanding of the early processes in the inner Solar System, of how our Earth was formed, how it evolved, and how it interacts with the Sun. A small, dense planet as the innermost outcome of a planetary formation process maybe followed by planetary migration should not be taken for granted. Up to date Mercury-sized planets have not yet been detected around other stars, hence the existence of exoplanetary systems similar to the Solar System in this respect, while likely, cannot be confirmed. On the other hand, there is a fast-growing list of exoplanetary systems with giant planets close to the central stars. Mercury has the most compressed magnetosphere due to the small dipole field and the short distance to the Sun. That allows the solar wind to reach and interact with the planetary surface directly due to the lack of an atmosphere. For an in-depth study of the planet and its environment, it is therefore necessary to operate spacecraft equipped with scientific instrumentation around the planet. BepiColombo will fly two spacecraft to Mercury addressing a wide range of scientific questions that will provide important clues on the origin and formation of terrestrial planets, its surrounding environment, and its interaction with the Sun.

Two spacecraft have visited Mercury so far. In 1974 and 1975 the Mariner 10 mission (e.g., Strom and Sprague 2003 and references herein) flew by Mercury three times, and in 2004 the MErcury Surface, Space ENvironment, GEochemistry, and Ranging (MESSENGER) spacecraft was launched. It completed four years of orbital observations on 30 April 2015 when it impacted on the surface of Mercury after running out of propellant (McNutt et al. 2004, 2006; Solomon et al. 2007).

Mariner 10 and MESSENGER made many new and, in various cases, unexpected observations, which require further follow-up investigation in order to piece together the evidence for the formation and evolution of the planet. Scientific and technical achievements 


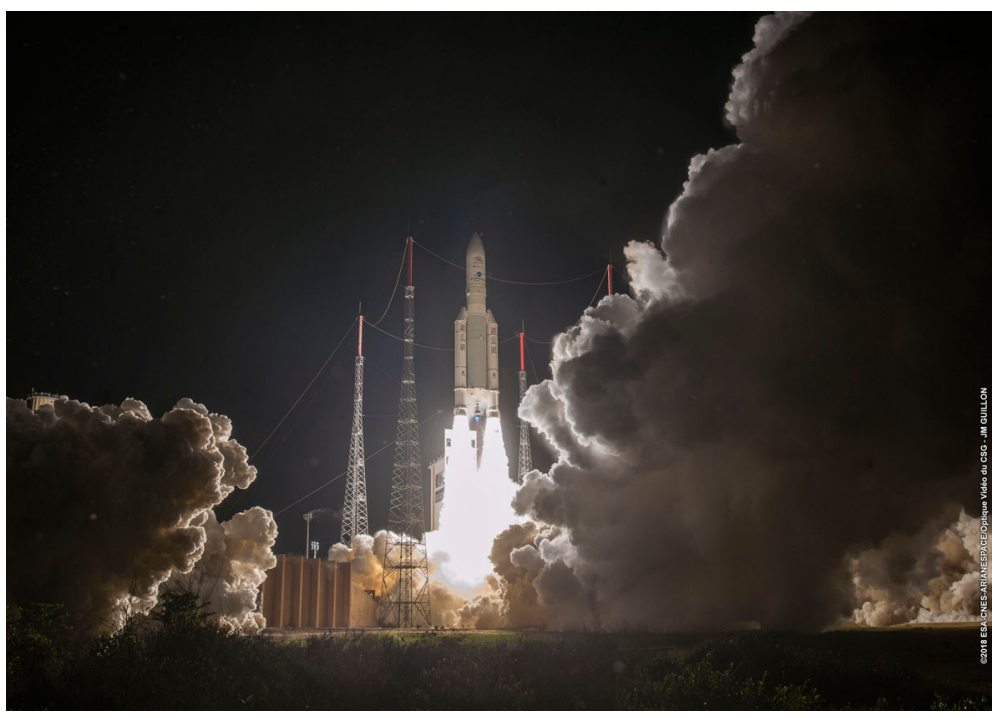

Fig. 1 BepiColombo liftoff on $20^{\text {th }}$ October 2018

are briefly reviewed in the instrument and science sections later. More details about the MESSENGER mission (Solomon and Anderson 2018) and the scientific achievements can be found in the MESSENGER book (Solomon et al. 2018).

The dual-spacecraft BepiColombo mission, developed jointly by the European Space Agency (ESA) and the Japan Aerospace Exploration Agency (JAXA) and launched on $20^{\text {th }}$ October 2018 (see Fig. 1) will follow-up on the achievements of these missions.

A comprehensive suite of state-of-art scientific instruments (Tables 2 and 3), flying on two spacecraft, allow a wide range of scientific questions to be addressed that will provide important clues on the origin and formation of terrestrial planets.

One spacecraft, the Mercury Planetary Orbiter (MPO), is led by ESA and its payload comprises eleven experiments and instrument suites. The MPO will focus on a global characterization of Mercury through the investigation of its interior, surface, exosphere, and magnetosphere. In addition, it will test Einstein's theory of general relativity.

The second spacecraft, the Mercury Magnetosphere Orbiter (MMO, since 2018 called $\mathrm{Mio}^{1}$ ) is led by JAXA and will carry five experiments or instrument suites to study the environment around the planet including the planet's exosphere and magnetosphere, and their interaction processes with the solar wind and the planet itself. During cruise both spacecraft are stacked together on top of the Mercury Transfer Module (MTM) propelled mainly by Solar Electric Propulsion (SEP). In addition the Mio spacecraft needs to be shielded by a Sunshield (MOSIF) during cruise (see Fig. 2). Upon arrival in late 2025, after a long journey of about seven years, the MTM will be jettisoned and chemical propulsion will be used to inject both spacecraft into their dedicated polar orbits. Mio will be released first, after which an additional thrust phase will insert the MPO into its final orbit. Both orbits are elliptical with eccentricity and inclination optimized for the study of Mercury and its magnetosphere (see Fig. 3).

\footnotetext{
${ }^{1}$ The nickname Mio of the Japanese spacecraft, MMO, was selected in 2018 as a result of a public contest.
} 


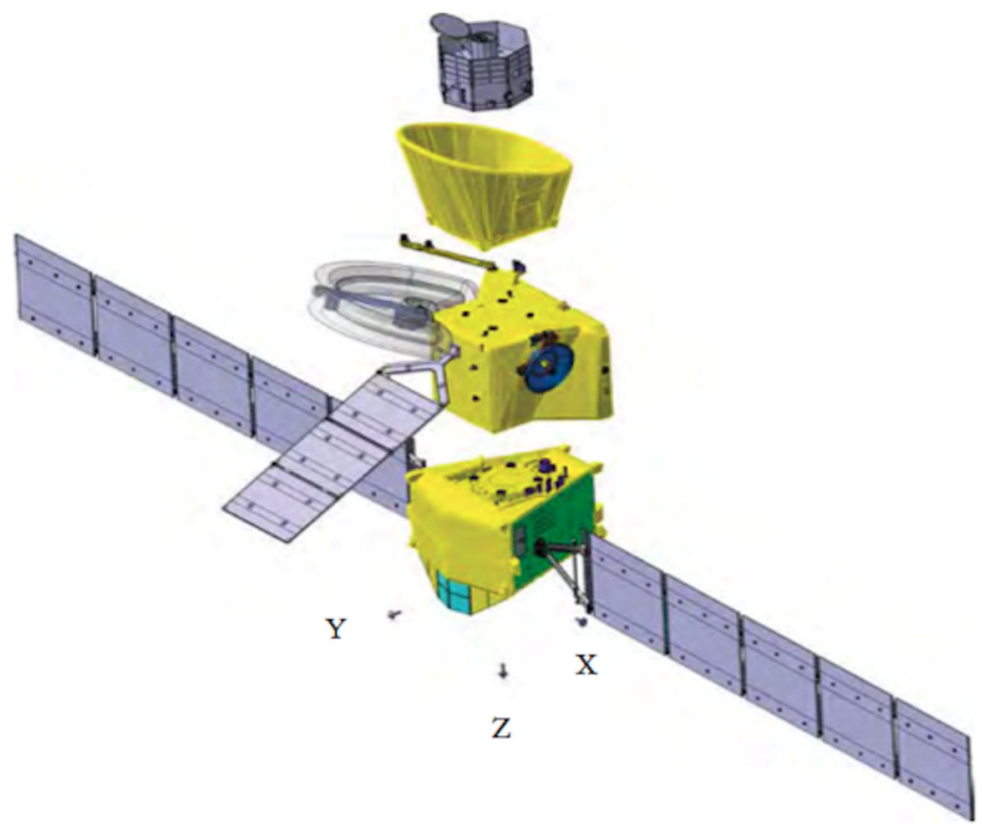

Fig. 2 Exploded view of the BepiColombo spacecraft stack, which consists of 4 separate elements (ordered here from launcher interface at the bottom to the top of stack): MTM, the Mercury Transfer Module, is an electric propulsion space tug which propels the stack to Mercury and provides power and attitude and orbit control during the cruise phase; $M P O$, the Mercury Planetary Orbiter, to study the planet surface and its interior. MOSIF, the MMO Sunshield and Interface Structure used to shield Mio during the cruise; and MMO nicknamed Mio, the Mercury Magnetospheric Orbiter; image provided by Airbus Defence and Space GmbH

The name BepiColombo was given to the mission in honor of Professor Giuseppe (Bepi) Colombo (1920-1984), best known for his work on the planet Mercury ((Colombo 1965; Anderson et al. 1987) and specifically for his support on placing NASA's Mariner 10 spacecraft into a resonant orbit with Mercury with multiple flybys (Beerer 1970). Colombo also explained Mercury's 3:2 spin-orbit resonance (Colombo and Shapiro 1966; Ward et al. 1976).

This article gives an overview of the BepiColombo mission history, the spacecraft composite and the mission profile. It briefly describes the suite of scientific instruments on board the two BepiColombo spacecraft and the science goals of the mission.

\section{Historical Background}

The first European proposal for a mission to visit Mercury was made to ESA in 1983 by Balogh and Richter. This mission was called Messenger, and the mission profile was somewhat similar to that of the NASA Mariner 10 mission. A multiple flyby mission with space physics objectives but also aimed of exploration of the inner heliosphere. However, it was clear from the start that an orbiter mission was needed rather than just another flyby mission. A Mercury Orbiter mission was suggested in response to an ESA call for new mission proposals on 10 July 1985 by Neukum and others (Balogh 2005). The mission design included a ballistic flight to Venus, a gravity assist at Venus, and 16 solar electric propulsion arcs 


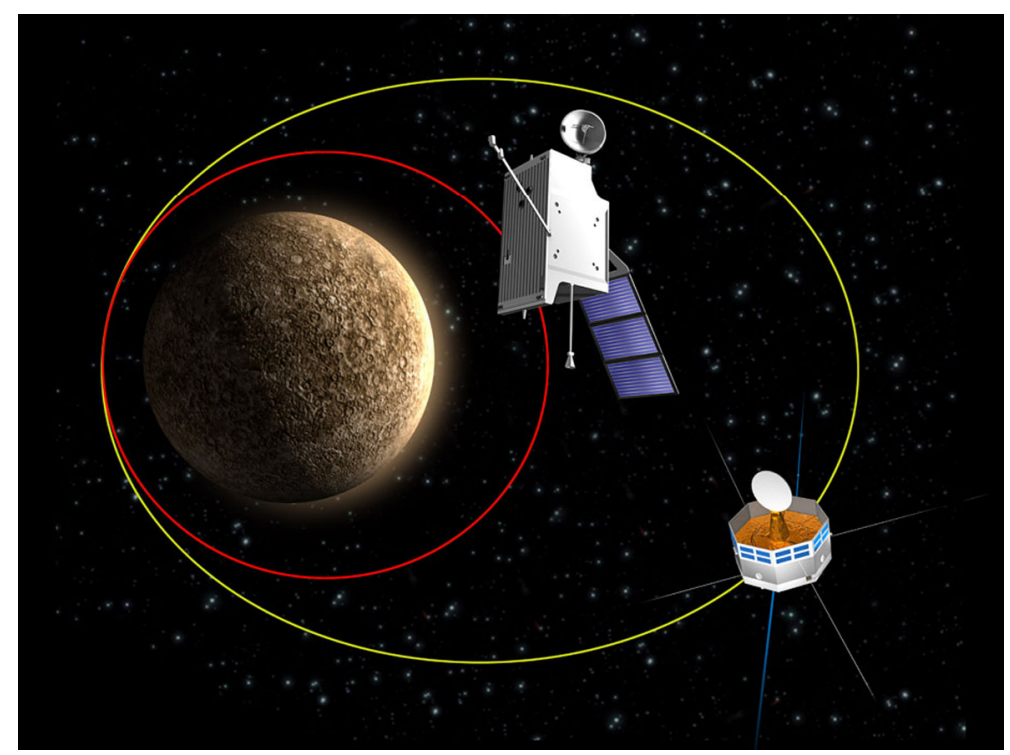

Fig. 3 Orbits of MPO $(480 \times 1,500 \mathrm{~km})$ and Mio $(590 \times 11,640 \mathrm{~km})$

to rendezvous Mercury with a final orbit at $300 \mathrm{~km}$ closest distance. The estimated flight time for this mission was 3.8 years with an anticipated launch in 1996. The discovery of a new class of gravity-assists missions by Yen (1985) and Yen (1989) using multiple-braking gravity-assist flybys at Earth, Venus, and Mercury led to a study of an dual orbiter mission for NASA (Belcher et al. 1991) at the end of the eighties. The two-spacecraft approach allowed simultaneous measurements at two different locations for determining the internal magnetic field of Mercury and to better characterise the environment around Mercury. However, this very complex mission was at the end not selected by NASA, but it was followed by other studies for a single orbiter mission, which resulted in the HERMES mission proposal in 1993 (Nelson et al. 1994, 2005) and the MESSENGER mission proposals in 1996 and 1998 (Solomon et al. 2001) in response to calls for discovery class missions.

In May 1993 a further Mercury orbiter mission, which then later became the BepiColombo mission, was proposed to ESA in response to a "Call for Ideas" (Balogh et al. 1994; Balogh 1994; Grard 1997). The mission was selected as a candidate in 1996 and finally in October 2000 was approved as the first cornerstone in ESA's Horizon 2000 Plus $^{2}$ science program, with a launch in 2009-2010 and a 3 years long cruise phase combining solar electric propulsion with planetary gravity assists. At that time BepiColombo consists of three major elements, the Mercury Planetary Orbiter, the Mercury Magnetospheric Orbiter (MMO), and a Mercury Surface Element (MSE). The original mission scenario involved separate launches of the three elements on two Soyuz-Fregat vehicles within the same launch slot from Baikonur, Russia (Grard and Balogh 2001; Novara 2002). In November 2001, the MSE was dropped from the mission baseline as the first studies revealed a much larger than expected impact on mass and budget (MSE required a Soyuz-Fregat launch by itself, the other Soyuz-Fregat carrying the MPO and MMO) at a time when the science program was under strong budgetary pressure. The restricted lift-off capability of the available launch ve-

\footnotetext{
${ }^{2}$ BepiColombo became the fifth mission of the combined Horizon 2000 and 2000 plus program of ESA.
} 
hicles at that time required very lightweight solutions for the scientific payload, the orbiters, and the propulsion systems needed to fly to Mercury. At the same time the spacecraft and instruments on-board had to survive the harsh thermal and radiation environment around Mercury and in the vicinity of the Sun. A clever mission scenario as well as technology developments that allowed saving propellant, i.e., highly efficient electric propulsion engines, were required. Advances in technology were also required to survive the very high temperatures at Mercury. During this phase the preliminary design was consolidated and critical technology was pre-developed (Balogh 2005; Jehn et al. 2004; Langevin 2000). A major milestone was achieved with the selection and successful implementation of SMART-1, an ESA technology demonstration mission, based on solar electric propulsion (SEP, Racca 1997 and which reached lunar orbit from a geostationary transfer orbit (GTO) using a combination of solar and lunar gravity assists in 2003-2006 (Foing et al. 2006).

On the JAXA side, a mission to Mercury was investigated by a newly formed Mercury Exploration Working Group in June 1997 under the Steering Committee for Space Science (SCSS) in the former Institute of Space and Astronautical Science (ISAS) (Yamakawa et al. 1996, 1999), which became part of JAXA in October 2003. The Mercury Exploration Working Group announced the Japanese plan of a spinning Mercury orbiter with chemical propulsion and multiple Venus and Mercury flybys. In November 1999 at the time of an Inter-Agency Consultative Group meeting, the possibility of collaboration with the ESA BepiColombo mission was discussed and expressed to ESA formally in July 2000. As a result, the International Mercury Exploration Mission in the framework of the BepiColombo program was approved by the SCSS of ISAS 18 months later followed by the formal approval by the Space Activities Commission in July 2003. On 6 November 2003, ESA's Science Programme Committee approved the BepiColombo mission with the MPO and the JAXA-provided MMO complement, which was named Mio shortly before launch, as part of their reconstructed Cosmic Vision Programme (Balogh 2005; Balogh et al. 2007).

The programmatic approval of Soyuz operations from Kourou (French Guiana) at that point in time, and the consequent increase in Soyuz launcher performance resulted in a new mission profile with a launch of both spacecraft on a single launcher, Soyuz-Fregat 2-1B vehicle, which was than approved as part of the ESA Cosmic Vision programme. The payload selection procedure for the MPO payload as outlined at the 105th meeting of the ESA Science Programme Committee on 6 November 2003 [reference document ESA/SPC 2003(41)] was unanimously approved. After a common Announcement of Opportunity between ESA and JAXA in 2004, 16 instruments, each lead by a Principal Investigator were selected and confirmed in 2005.

In 2008 the BepiColombo mission experienced a severe mass crisis caused among other factors by negative and unexpected test results on mission essential technologies (e.g., strong solar array performance degradation at high temperatures and the harsh ultraviolet radiation environment expected at Mercury). This resulted in a much larger area of the solar panels and a more robust structure, more fuel consumption etc., and at the end, due to the associated increase in spacecraft mass, also a change of the launch vehicle to Ariane 5. At the end, more than $80 \%$ of the technologies and material used on BepiColombo were either newly developed or had to be qualified to function around Mercury; for example the solar arrays, the high gain antenna, the multi-layer insulation and lubricants and coatings being adapted to function in this harsh environment. The final approval of the redesigned mission was made in November 2009 (Benkhoff et al. 2010). In the following years, unexpected test results during a thermal balance test of MPO and a short circuit in a relay in the MTM power processing unit caused further delays. Finally on $20^{\text {th }}$ October 2018 the BepiColombo mission was successfully launched from the European spaceport in Kourou. 
Although initially not planned in that way, the sequenced approach that BepiColombo will arrive in an orbit around Mercury about a decade and a half later than NASA's discovery class mission MESSENGER has many benefits from a scientific point of view. BepiColombo will follow up and complement the work of MESSENGER by providing new, comprehensive scientific data sets. In addition, the two spacecraft of BepiColombo will add dual point measurements within Mercury's environment and observations from instruments not on-board MESSENGER. Nevertheless, partly similar payloads on BepiColombo and MESSENGER (Solomon et al. 2001) are a desirable feature that will generate significant complementary and synergistic opportunities between the two missions. From the beginning, there was a close cooperation and scientific exchange between NASA's MESSENGER and the ESA-JAXA BepiColombo teams. As a result, BepiColombo has benefited from findings and scientific results from MESSENGER (Solomon et al. 2018) to make the mission a perfect candidate for the next giant step of Mercury explorations.

\section{The BepiColombo mission}

BepiColombo is dedicated to the thorough exploration of Mercury and its environment. With its two-spacecraft, interdisciplinary approach, the BepiColombo mission will provide the detailed information necessary to understand the process of planetary formation and evolution in the hottest part of the proto-planetary nebula, as well as the similarities and differences between the magnetospheres of Mercury and Earth. The BepiColombo mission design is driven essentially by the scientific payload requirements, the launch mass constraints, and the harsh thermal and radiation environment at Mercury. Key technologies required for the implementation of this challenging mission include the following:

- High-temperature thermal control materials (coatings, adhesives, resins, multi-layer insulation blankets (MLI), Optical solar reflectors (OSR))

- Radiator design for high-infrared environment

- High-temperature and high-intensity solar cells, diodes, and substrates for the solar arrays

- High-temperature steerable high-gain and medium gain antennas

- High specific impulse (Isp = $3800 \mathrm{~s}$ ) and high total impulse (17 MNs), to be provided by gridded ion engines.

- Payload technology, such as detectors, filters, and laser technology.

The total spacecraft mass is $4,043 \mathrm{~kg}$ of which $1411 \mathrm{~kg}$ are propellants, see Table 1 . Despite travelling towards the Sun, the transfer module requires a large solar array as a consequence of the design in order to keep the overall temperature of the panels below of about $200^{\circ}$ Celsius. The two wings of the transfer module total about $42 \mathrm{~m}^{2}$ and have to be rotated away from the Sun to avoid overheating. Similar design rules are applied to the MPO solar panel, which has a length of about $7.5 \mathrm{~m}$. The size of the MPO radiator was limited in order to be compatible with the launcher fairing diameter. The thermal design drivers for the MPO and Mio are the Mercury approach phase and the Mercury orbit phase where extremely high solar and planetary fluxes will occur. Given the high eccentricity of Mercury's orbit the solar radiance is a function of Mercury's true anomaly, varying from $6290 \mathrm{~W} / \mathrm{m}^{2}$ at Mercury aphelion to $14500 \mathrm{~W} / \mathrm{m}^{2}$ at Mercury perihelion. The thermal design of the MPO was a major design driver for the BepiColombo mission and is discussed in greater depth in Ferrero et al. (2016). 
Table 1 BepiColombo: Spacecraft mass by subsystem

\begin{tabular}{|c|c|c|}
\hline Element & Subsystem & Mass $(\mathrm{kg})$ \\
\hline \multirow[t]{13}{*}{ MPO } & Payload & 98.2 \\
\hline & Structure & 284.8 \\
\hline & Mechanisms & 27.6 \\
\hline & Thermal & 168.9 \\
\hline & Power & 141.7 \\
\hline & Data Management & 40.1 \\
\hline & Attitude \& Orbit Control & 63.5 \\
\hline & Chemical Propulsion & 79.4 \\
\hline & Communications & 154.5 \\
\hline & Harness & 87.9 \\
\hline & Dry mass total & $1,146.6$ \\
\hline & Propellant & 673.2 \\
\hline & Total element mass & $1,819.8$ \\
\hline \multirow[t]{4}{*}{ Mio } & Payload (incl. harness & 40.0 \\
\hline & Mio spacecraft total & 255.0 \\
\hline & Interface $\mathrm{H} / \mathrm{W}$ & 19.7 \\
\hline & Total element mass & 274.7 \\
\hline \multirow[t]{5}{*}{ MOSIF } & Structure & 85.8 \\
\hline & Mechanisms & 8.0 \\
\hline & Thermal & 25.5 \\
\hline & Harness & 1.5 \\
\hline & Total element mass & 120.8 \\
\hline \multirow[t]{13}{*}{ MTM } & Structure & 262.2 \\
\hline & Mechanisms & 16.5 \\
\hline & Thermal & 87.1 \\
\hline & Power & 312.8 \\
\hline & Data Management & 10.4 \\
\hline & Attitude \& Orbit Control & 1.2 \\
\hline & Chemical Propulsion & 39.3 \\
\hline & Electrical Propulsion & 286.8 \\
\hline & Harness & 67.4 \\
\hline & Miscellanea & 6.5 \\
\hline & Dry mass total & $1,090.2$ \\
\hline & Propellant & 737.7 \\
\hline & Total element mass & $1,827.9$ \\
\hline \multirow[t]{3}{*}{ BepiColombo } & Dry mass total & $2,632.3$ \\
\hline & Propellant & $1,410.9$ \\
\hline & Total spacecraft mass & $4,043.2$ \\
\hline
\end{tabular}




\subsection{Mercury Planetary Orbiter (MPO)}

The BepiColombo MPO accommodates the 11 scientific instruments (Table 2) and has a box-like shape with a size of $3.9 \times 2.2 \times 1.7 \mathrm{~m}$ (Fig. 4). The entire MPO totals up to $1146.6 \mathrm{~kg}$ of nominal dry mass (Table 1). A specific primary double-H structure allows most payloads to look through the hot=planet-facing side, while being mounted on inner walls cooled by heat-pipes, ensuring a good accessibility during the integration process. The primary structure carries an external thin cage structure to which the high temperature MLI (Multi-Layer-Insulation) is fixed. In the center of the MPO two tanks are placed, carrying the propellant for the propulsion system. The MPO is designed to take scientific measurements in all parts of the orbit throughout the Mercury year, implying that most of the apertures of the remote sensing instruments are continuously nadir pointing. As a consequence, 5 out of 6 spacecraft faces may be illuminated by the Sun at some point. This leaves only one spacecraft side for a radiator to dump excess heat into space and to avoid solar exposure of the radiator. A further consequence is a spacecraft flip-over manoeuvre, which is needed twice per Mercury year. The heat load is tremendous: At the perihelion the solar flux is about $14 \mathrm{~kW} / \mathrm{m}^{2}$ and the thermal flux is about $6 \mathrm{~kW} / \mathrm{m}^{2}$ at the equator, when the spacecraft is close to apoherm (altitude: $1550 \mathrm{~km}$ ). This environment imposes strong requirements on the spacecraft design, particularly to all elements that are exposed to the Sun and Mercury, such as the solar array, mechanisms, antennae, multi-layer insulation, and thermal coatings. The development of these elements, together with the SEP system, were the main cost drivers for this mission and at the same time are responsible for a sizable share of the overall spacecraft mass. Figure 7 shows the schematic block diagram for the MPO and MTM spacecraft. Details can be found in the following sections.

\subsubsection{Power}

The average power demand of the MPO in Mercury orbit, when conducting scientific measurements, is on the order of $1140 \mathrm{~W}$ (Table 4, column: Science Phase General). This is provided via a $28 \mathrm{~V}$ regulated power bus by the solar array and a battery during eclipse phases. The solar array is a single 3-panel wing with one side covered with solar cells. Due to the high intensity of the solar irradiation, the solar array has a $70-30 \%$ mixture of cells and Optical Surface Reflectors (OSR) for keeping the temperature within the allowed limits. The solar array needs to track the Sun continuously in order to keep its temperature below $200{ }^{\circ} \mathrm{C}$. This is supported by choosing Sun angles of incidence up to $84^{\circ}$ that generate enough power but do not unnecessarily heat up the solar array.

\subsubsection{Thermal}

One of the biggest challenges in going to Mercury is the thermal environment. The intensity of the solar irradiation is up to 10 times higher than at Earth with a maximum of about $14 \mathrm{~kW} / \mathrm{m}^{2}$. In addition, at MPO orbital altitude the $\mathrm{S} / \mathrm{C}$ receives up to $5400 \mathrm{~W} / \mathrm{m}^{2}$ of energy flux density emitted as infrared radiation by the planet and up to $600 \mathrm{~W} / \mathrm{m}^{2}$ of sunlight reflected by the planet. This has two important consequences for the spacecraft design: First, all surfaces, units, payloads, appendages etc., exposed to this environment must be able to withstand high temperatures. Second, as far as possible, the irradiation needs to be reflected and not absorbed. The outer surface of the MLI has a low solar absorptivity to reflect most of the sunlight. Nevertheless, it heats up to more than $360{ }^{\circ} \mathrm{C}$, which prevents the utilization of standard MLI, and requires a ceramic fabric with titanium layers. Low absorptivity is also 
Table 2 Instruments on the BepiColombo Mercury Planetary Orbiter, MPO

\begin{tabular}{|c|c|c|c|}
\hline Instrument & & Observational Objective & $\begin{array}{l}\text { Principal } \\
\text { Investigator } \\
\text { (Country) }\end{array}$ \\
\hline BELA & $\begin{array}{l}\text { BepiColombo Laser } \\
\text { Altimeter }\end{array}$ & $\begin{array}{l}\text { Characterize the } \\
\text { topography and surface } \\
\text { morphology of Mercury. }\end{array}$ & $\begin{array}{l}\text { Hauke Hussmann } \\
\text { (Germany) } \\
\text { Nicolas Thomas } \\
\text { (Switzerland) }\end{array}$ \\
\hline MORE & $\begin{array}{l}\text { Mercury Orbiter } \\
\text { Radio Science } \\
\text { Experiment }\end{array}$ & $\begin{array}{l}\text { Determine Mercury's } \\
\text { gravity field as well as the } \\
\text { size and physical state of } \\
\text { its core. }\end{array}$ & $\begin{array}{l}\text { Luciano Iess } \\
\text { (Italy) }\end{array}$ \\
\hline ISA & $\begin{array}{l}\text { Italian Spring } \\
\text { Accelerometer }\end{array}$ & $\begin{array}{l}\text { Study Mercury's interior } \\
\text { structure and test } \\
\text { Einstein's theory of } \\
\text { relativity. }\end{array}$ & $\begin{array}{l}\text { Valerio Iafolla } \\
\text { (Italy) }\end{array}$ \\
\hline MPO-MAG & $\begin{array}{l}\text { Mercury } \\
\text { Magnetometer }\end{array}$ & $\begin{array}{l}\text { Describe Mercury's } \\
\text { magnetic field and its } \\
\text { source. }\end{array}$ & $\begin{array}{l}\text { Daniel Heyner } \\
\text { (Germany) }\end{array}$ \\
\hline MERTIS & $\begin{array}{l}\text { Mercury Thermal } \\
\text { Infrared Spectrometer }\end{array}$ & $\begin{array}{l}\text { Determine Mercury's } \\
\text { mineralogical } \\
\text { composition and obtain a } \\
\text { global map of the surface } \\
\text { temperature. }\end{array}$ & $\begin{array}{l}\text { Harald Hiesinger } \\
\text { (Germany) }\end{array}$ \\
\hline MGNS & $\begin{array}{l}\text { Mercury Gamma-ray } \\
\text { and Neutron } \\
\text { Spectrometer }\end{array}$ & $\begin{array}{l}\text { Determine the elemental } \\
\text { composition of Mercury's } \\
\text { surface distribution of } \\
\text { volatiles in the polar areas. }\end{array}$ & $\begin{array}{l}\text { Igor Mitrofanov } \\
\text { (Russia) }\end{array}$ \\
\hline MIXS & $\begin{array}{l}\text { Mercury Imaging } \\
\text { X-ray Spectrometer }\end{array}$ & $\begin{array}{l}\text { Obtain a global map of the } \\
\text { surface atomic } \\
\text { composition. }\end{array}$ & $\begin{array}{l}\text { Emma Bunce } \\
\text { (Great Britain) }\end{array}$ \\
\hline PHEBUS & $\begin{array}{l}\text { Probing of Hermean } \\
\text { Exosphere by } \\
\text { Ultraviolet } \\
\text { Spectroscopy }\end{array}$ & $\begin{array}{l}\text { Characterize the } \\
\text { composition and } \\
\text { dynamics of Mercury's } \\
\text { exosphere. }\end{array}$ & $\begin{array}{l}\text { Eric Quémerais } \\
\text { (France) }\end{array}$ \\
\hline SERENA & $\begin{array}{l}\text { Search for Exosphere } \\
\text { Refilling and Emitted } \\
\text { Neutral Abundances }\end{array}$ & $\begin{array}{l}\text { Study the interactions } \\
\text { among the surface, } \\
\text { exosphere, } \\
\text { magnetosphere, and the } \\
\text { solar wind. }\end{array}$ & $\begin{array}{l}\text { Stefano Orsini } \\
\text { (Italy) }\end{array}$ \\
\hline SIMBIO-SYS & $\begin{array}{l}\text { Spectrometers and } \\
\text { Imagers for MPO } \\
\text { Integrated } \\
\text { Observatory System }\end{array}$ & $\begin{array}{l}\text { Provide global, } \\
\text { high-resolution, and } \\
\text { infrared imaging of the } \\
\text { surface. }\end{array}$ & $\begin{array}{l}\text { Gabriele Cremonese } \\
\text { (Italy) }\end{array}$ \\
\hline SIXS & $\begin{array}{l}\text { Solar Intensity X-ray } \\
\text { and particle } \\
\text { Spectrometer }\end{array}$ & $\begin{array}{l}\text { Perform measurements of } \\
\text { solar X-rays and energetic } \\
\text { particles at high time } \\
\text { resolution. }\end{array}$ & $\begin{array}{l}\text { Juhani Huovelin } \\
\text { (Finland) }\end{array}$ \\
\hline
\end{tabular}



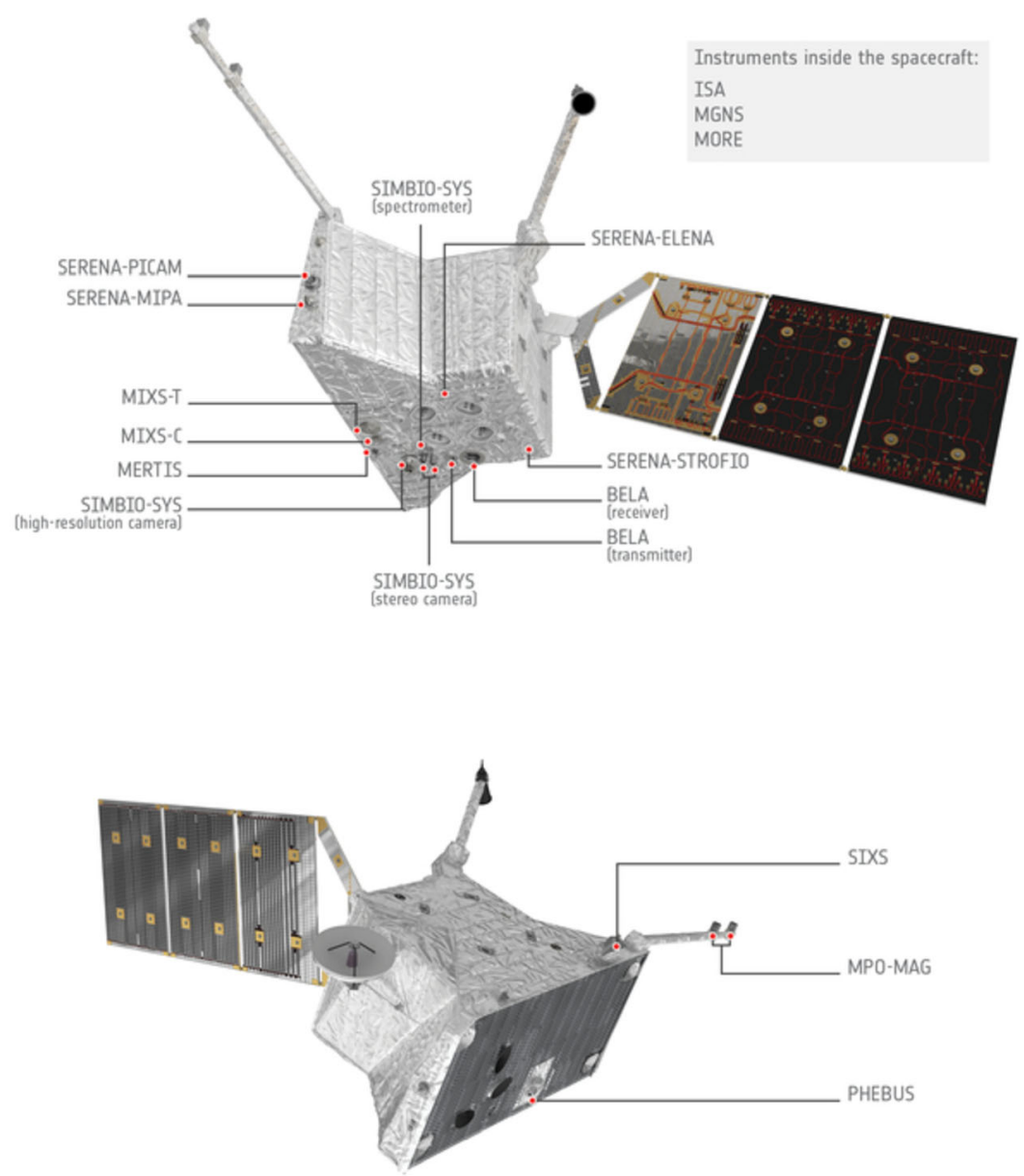

Fig. 4 MPO and instrument positions

used for the high gain antenna coating, which is, due to its position, fully exposed to the Sun, albedo and infrared. In addition, the radio science experiment asks for a very stable antenna, which was implemented largely in titanium. Although the radiator is not exposed to direct sunlight, it will receive intense albedo and infrared from Mercury itself. To minimize the influence of this parasitic heat flux on the radiator, highly reflective fins (polished and geometrically reflecting outwards) have been mounted to it at an appropriate angle, to minimize absorption of heat radiated from Mercury, while at the same time allowing radiation towards deep space. Inside the spacecraft, the temperatures are kept within the standard range $\left(0{ }^{\circ} \mathrm{C}\right.$ $-50{ }^{\circ} \mathrm{C}$ ) and for specific instruments, interface temperatures below $-10{ }^{\circ} \mathrm{C}$ are provided. The transportation of the heat is accomplished by heat pipes embedded in the double- $\mathrm{H}$ panels and the radiator. This yields an even temperature distribution within the spacecraft. The radiator is divided into separate segments, allowing the generation of different interface temperatures and temperature stabilities. Heaters are used in the coldest phases of the Mercury year. 


\subsubsection{Communications and Tracking}

Communication with Earth is ensured via a high gain antenna (HGA), a medium gain antenna (MGA), and two low gain antennas (LGA). The MGA is located on a boom and provides a wide geometrical coverage during nearly the entire mission, ensuring that contact is maximized with respect to spacecraft attitude and Earth position. The MGA is used during cruise and orbit injection manoeuvres at Mercury, as well as in case of safe modes, when the high gain antenna may not be available. The HGA provides a link with high data rate for science data transmission. This is achieved by sending the data to Earth in $\mathrm{X}$ and Ka-band in parallel. Over the time period of one Earth year in Mercury orbit, more than 1550 Gbit can be downlinked to Earth. The HGA also provides the means for the MORE radio science experiment based on the up- and downlink in both frequency bands. This allows very precise range and range rate (Doppler) measurements resulting in a very accurate orbit determination.

\subsubsection{Attitude Control}

The control of the attitude is provided by a set of four reaction wheels and 4 redundant $5 \mathrm{~N}$ thrusters for wheel de-saturation. Three star trackers, Sun sensors, and a high precision gyroscope package are employed as sensors in the attitude control. The combination of the star trackers and gyroscopes information into a gyro-stellar attitude estimator ensures a very precise attitude determination (on the order of a few arcseconds), which is required by several experiments. Since the pointing requirement for efficient data downlink must be in the order of arcminutes, it is important to minimise the solar irradiation of sensitive surfaces. In case of a failure that entails a loss of attitude leading to an entry into survival mode. The control concept for the survival mode is selected in such a way that in no case the sensitive surfaces will be exposed to the Sun for longer than $85 \mathrm{~s}$, and it is based on the use of gyroscope information. In addition, the Sun sensors are also used in the attitude estimator during an extended-duration survival mode, when the required body axis is to be kept aligned with the Sun direction.

\subsubsection{Propulsion}

The propulsion system of the MPO consists of 4 redundant $22 \mathrm{~N}$ thrusters in the nadir face of the spacecraft that are only employed in orbital manoeuvres until final orbit acquisition, after which they will be passivated. For reaction wheel de-saturation and attitude control, four redundant $5 \mathrm{~N}$ thrusters are mounted on the radiator. The propellant is stored in two main tanks (plus two smaller auxiliary tanks) in the centre of the spacecraft containing hydrazine as fuel and Mixed Oxides of Nitrogen (MON) as oxidiser. The $22 \mathrm{~N}$ thrusters are operated in a bi-propellant mode using hydrazine and MON, yielding a high specific impulse, whereas the $5 \mathrm{~N}$ thrusters operate with hydrazine only, which minimises contamination by the exhaust plume. Such a system is called a "dual mode" system, since it combines bi-propellant and monopropellant based operation modes (Sutherland et al. 2019).

\subsection{Mio: Mercury Magnetospheric Orbiter (MMO)}

The BepiColombo Mio is a spin-stabilized spacecraft (Fig. 5) after it is separated from the MPO following the Mercury orbit insertion. The Mio is optimized for in situ plasma and electromagnetic fields and waves measurements in Mercury orbit. The nominal spin rate is 


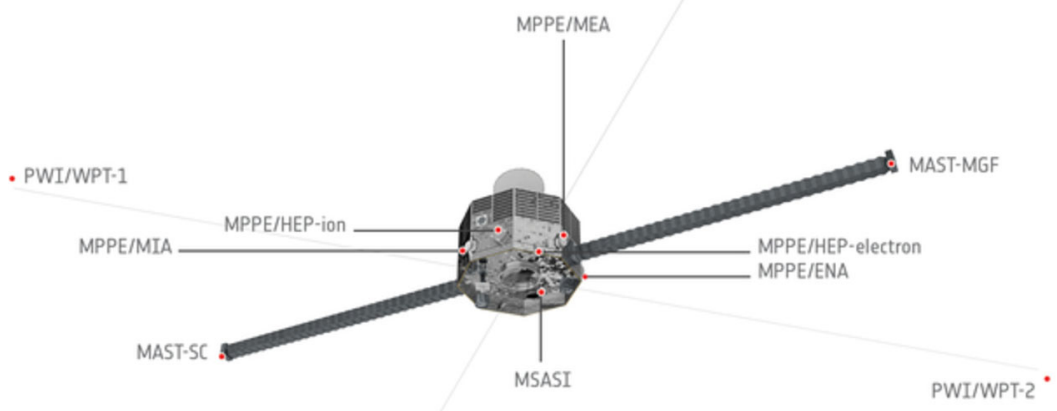

PWI/MEFISTO-1.

Fig. 5 Mio and Mio instrument positions

$15 \mathrm{rpm}$ (spin period of $4 \mathrm{~s}$ ) due to the scientific requirements. The spin axis is pointed nearly perpendicular to the Mercury orbital plane. The total mass of Mio is $255 \mathrm{~kg}$, including $\mathrm{N}_{2}$ (nitrogen) gas for attitude control. The Mio main structure consists of two decks (upper and lower), a central cylinder (thrust tube), and four bulkheads. The external appearance has an octagonal shape, which can be surrounded by a $1.8 \mathrm{~m}$ diameter circle. The height of the side panel is $1.1 \mathrm{~m}$, whose upper portion is covered to $70 \%$ by solar cells and to $30 \%$ by optical solar reflectors (OSR). The lower portion is covered by OSRs only. The instruments (Table 3) are located on the upper and lower decks whose separation is $40 \mathrm{~cm}$ (Yamakawa et al. 2005a,b). Figure 6 shows the schematic block diagram for MPO spacecraft. Details can be found in the following sections.

\subsubsection{Power}

During the interplanetary cruise phase, MPO provides the heater power, since the Mio spacecraft is not exposed to the direct solar flux owing to the Mio sunshield. Mio is equipped with the primary heaters controlled by the Mio heater control electronics as well as the survival heater with thermostats. After Mio separation, the solar cells will be exposed to wide temperature variations due to the variation of Mercury's distance from the Sun $(0.31-0.47 \mathrm{AU})$. The bus voltage is $50 \mathrm{~V}$. The multi-junction cell with conductive coating type cover glass is used as the solar cell. A Li-Ion secondary type battery with nameplate capacity of $23.5 \mathrm{Ah}$ is designed to cope with the $2 \mathrm{hrs}$ maximum eclipse condition around Mercury. All external surfaces have high electrical conductivity in order to keep the surface at the same electric potential with respect to the environment, which is essential to DC electric field and lowenergy electron measurements (see Fig. 6). 
Table 3 Instruments on the BepiColombo Mercury Magnetospheric Orbiter, Mio

\begin{tabular}{|c|c|c|c|}
\hline Instrument & & Observational Objective & $\begin{array}{l}\text { Principal } \\
\text { Investigator } \\
\text { (Country) }\end{array}$ \\
\hline MGF & $\begin{array}{l}\text { Mercury } \\
\text { Magnetometer }\end{array}$ & $\begin{array}{l}\text { Provide a detailed description of } \\
\text { Mercury's magnetosphere and of } \\
\text { its interaction with the planetary } \\
\text { magnetic field and the solar wind. }\end{array}$ & $\begin{array}{l}\text { Wolfgang } \\
\text { Baumjohann } \\
\text { (Austria) }\end{array}$ \\
\hline MPPE & $\begin{array}{l}\text { Mercury Plasma } \\
\text { Particle } \\
\text { Experiment }\end{array}$ & $\begin{array}{l}\text { Study low- and high-energy } \\
\text { particles in the magnetosphere. }\end{array}$ & $\begin{array}{l}\text { Yoshifumi Saito } \\
\text { (Japan) }\end{array}$ \\
\hline PWI & $\begin{array}{l}\text { Plasma Wave } \\
\text { Instrument }\end{array}$ & $\begin{array}{l}\text { Make a detailed analysis of the } \\
\text { structure and dynamics of the } \\
\text { magnetosphere. }\end{array}$ & $\begin{array}{l}\text { Yasumasa } \\
\text { Kasaba (Japan) }\end{array}$ \\
\hline MSASI & $\begin{array}{l}\text { Mercury Sodium } \\
\text { Atmospheric } \\
\text { Spectral Imager }\end{array}$ & $\begin{array}{l}\text { Measure the abundance, } \\
\text { distribution, and dynamics of } \\
\text { sodium in Mercury's exosphere. }\end{array}$ & $\begin{array}{l}\text { Ichiro } \\
\text { Yoshikawa } \\
\text { (Japan) }\end{array}$ \\
\hline MDM & $\begin{array}{l}\text { Mercury Dust } \\
\text { Monitor }\end{array}$ & $\begin{array}{l}\text { Study the distribution of } \\
\text { interplanetary dust in the orbit of } \\
\text { Mercury. }\end{array}$ & $\begin{array}{l}\text { Masanori } \\
\text { Kobayashi } \\
\text { (Japan) }\end{array}$ \\
\hline
\end{tabular}

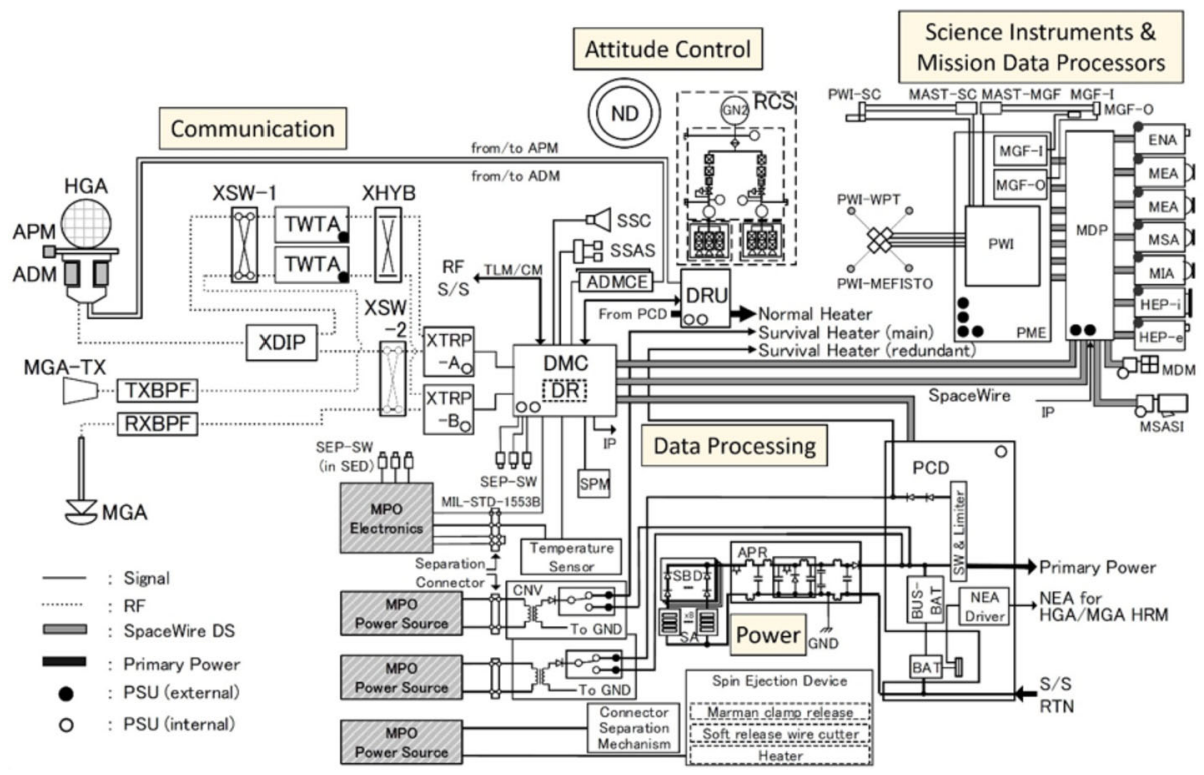

Fig. 6 MPO and MTM Block Diagram 


\subsubsection{Thermal}

Mio, being a spinning $\mathrm{S} / \mathrm{C}$, is controlled by a passive thermal design combined with an actively controlled heater system for the coldest phases of the mission to maintain the onboard equipment and the spacecraft structure within the proper temperature range. The passive control elements are the OSR, thermal shield, paints, films, and multilayer insulation blankets (MLI). The internal surfaces of the upper and lower deck have high emissivity surfaces (black paint) to equalize internal temperature. The external surface of the upper deck is covered in MLI for insulation from the external thermal environment whereas the external surface of the lower deck functions as a radiator and is covered by OSRs to have low absorptivity and high emissivity. The octagonal prism is divided into three parts; upper, middle, and lower prisms. The external surface of the upper prism is covered in solar cells and OSRs at a 70:30 ratio. OSRs are put on the internal surface of the upper prism to reduce the cell temperature. The middle prism has OSRs on the exterior and MLI on the interior. The complete $\mathrm{S} / \mathrm{C}$ is kept inclined at 9 degrees for the reduction of the sunlight reflection to the lower deck, and it has a low $-\alpha /$ low $-\epsilon$ finish to reflect the direct solar flux and to reduce heat transfer by radiation from the lower prism to the lower deck radiator. The octagonal prism (substrate) is insulated from the upper and lower decks with thermal standoffs. Most of the internal components have a surface with high emissivity (black paint) to equalize the internal temperature. The temperatures of the batteries are controlled independently with the aid of radiators and heaters, which are installed on a battery panel. This panel is attached to the bottom of the central cylinder and insulated from the main structure by MLI and thermal standoffs. The radiator has a surface with high emissivity OSRs (Optical Solar Reflectors). The antenna de-spun motor (ADM) and the gaseous nitrogen (GN2) tank are covered with MLI for insulation from the external thermal environment. The HGA disks are painted white. For more details see Ogawa et al. (2015).

\subsubsection{Communication and Tracking}

An $80 \mathrm{~cm}$ diameter HGA is used for the high rate X-band telemetry (TLM) /command (CM) and ranging link, with the use of a $20 \mathrm{~W}$ power amplifier. The Mio HGA is pointed towards the Earth with the ADM and antenna pointing mechanism (APM) for the elevation angle control of $-13^{\circ}$ to $+13^{\circ}$ determined by the geometry of the planets' orbits. A MGA is accommodated for emergency TLM/CM link. The MGA is installed on the lower surface of the Mio and will be deployed after the separation from the remaining spacecraft composite. In Mercury's orbit, the Mio's telemetry rate will change as a function of the distance from the Earth. The average bit rate of the HGA is $16 \mathrm{kbs}^{-1}$, which in turn translates into $40 \mathrm{MB} /$ day.

\subsubsection{Attitude Control}

The spin-stabilized Mio spacecraft attitude will be measured by a pair of Sun sensors on the side panel and a star scanner attached to the bottom surface. The attitude is controlled by the propulsion system with cold gas jets. A nutation damper installed inside the central cylinder is used for passive nutation damping. The Mio propulsion system uses a cold gas jet system, since only attitude control capability is required (i.e., no orbit control function). It consists of one propellant tank, six $0.4 \mathrm{~N}$-class nitrogen gas (GN2) jet thrusters, valves, piping, and thermal control equipment (heaters and sensors). The four tangential thrusters for roll control are allocated on the side panel, while the two axial thrusters are mounted at the bottom of the spacecraft body. The GN2 tank consists of a titanium alloy liner and 
a carbon fibre shell. The tank volume is 14.9 liters and the maximum designed pressure is $27.7 \mathrm{MPa}$. About $3.69 \mathrm{~kg}$ of GN2 is loaded including residual propellant of $0.69 \mathrm{~kg}$. The downstream part of the propulsion system consists of a fully redundant system.

For the HGA, a helical array antenna of $80-\mathrm{cm}$ diameter excited by the radial line is mounted on top of Mio. The HGA is pointed toward the Earth by the ADM and an elevation control mechanism, the APM. As for the MGA (medium gain antenna), a bi-reflector type receiver antenna is mounted on the lower surface with a deployment mechanism, and a horntype transmitting antenna is mounted on the upper deck. Most of the scientific instruments (particle sensors, etc.) are allocated on the upper side of the bottom deck, while the four deployment units of electric probe antennas for PWI are installed on the lower side of the bottom deck. For more information and further details see Murakami et al. (2020), Kasaba et al. (2020b).

\subsection{MTM: Mercury Transfer Module}

The MTM provides the acceleration and braking required during cruise to reach the eventual capture by Mercury and the large amount of power required by the solar electric propulsion system. The MTM also constitutes the bottom element in the overall spacecraft stack (Kempkens et al. 2019).

Figure 8 shows the MTM with its 2 solar array wings totaling over $40 \mathrm{~m}^{2}$, which provide the power for the SEPS (Solar Electric Propulsion System) during cruise.

The MTM is equipped with a bi-propellant propulsion system of 12 redundant 10-N thrusters that are used for the attitude control activities during cruise. The bi-propellant system is also able to provide navigation $\Delta V$ manoeuvres during cruise. By far the major part of the $\Delta V$ required during cruise is delivered by the SEPS, using its four $125 \mathrm{mN}$ ion thrusters that are initially operated singly and later in pairs (Gray et al. 2019; Lewis et al. 2015; Sutherland et al. 2019). The MTM solar arrays use the same high-temperature technologies as developed for the MPO and are rotated away from the Sun for the purpose of temperature control. At their peak output the MTM solar array delivers $13 \mathrm{~kW}$, of which $10.3 \mathrm{~kW}$ is required by the SEPS (see Table 4 column 2: Cruise Phase Thrust Mode).

To accomplish thermal control, the MTM thermal design incorporates a novel sunshield using a special high temperature Multi Layer Insulation (MLI) plus a radiator with an area of about $9 \mathrm{~m}^{2}$. When exposed to the $14.5 \mathrm{~kW} / \mathrm{m}^{2}$ solar flux at about $0.3 \mathrm{AU}$ internal temperatures must be suitable for the operation of any two of its four $\sim 5 \mathrm{~kW}$ electric thrusters simultaneously, while at the same time maintaining 12 redundant bi-propellant reaction control thrusters, internal electronic equipment within standard temperature ranges, thruster pointing mechanisms, and a xenon propulsion system (see Fig. 7. In addition, the design must still allow significant flexibility for its orientation to the Sun and emergency situations (for example the ability to survive short-term losses of attitude). More details on the thermal design of the MTM can be found in Tuttle and Cavallo (2009).

The MTM Structure is based on a CFRP (Carbon Fibre Reinforced Plastic) conical primary structure interfacing with the Launch Vehicle Adapter and the MPO. The mechanical interfaces to MPO are characterised by cup-cone separation systems for in-flight separation 7.2 years after launch whilst initially providing the primary load path through the MCS (Mercury Composite Spacecraft) structure at launch.

\subsection{MMO sunshield and Interface Structure, MOSIF}

The MOSIF is the MMO sunshield and Interface Structure (see Fig. 9). As the name suggests, it provides the interface structure between the MPO and Mio and protects Mio from the full intensity of the Sun until its separation - having reached its operational orbit. 
Table 4 BepiColombo: Spacecraft power demand by module and by subsystem, in watts. Given are examples for different scenarios during cruise (with and without SEP thrust) and in MPO orbit (full power mode or reduced around perihelion)

\begin{tabular}{|c|c|c|c|c|c|}
\hline & $\begin{array}{l}\text { Cruise Phase } \\
\text { Coast Mode }\end{array}$ & $\begin{array}{l}\text { Cruise Phase } \\
\text { Thrust Mode }\end{array}$ & $\begin{array}{l}\text { Science Phase } \\
\text { General }^{\mathrm{c}}\end{array}$ & $\begin{array}{l}\text { Science Phase } \\
\text { Perihelion }^{\mathrm{d}}\end{array}$ & $\begin{array}{l}\text { Science Phase } \\
\text { Perihelion }^{\mathrm{c}}\end{array}$ \\
\hline \multicolumn{6}{|l|}{ MPO } \\
\hline Payload & 45.2 & 23.1 & 194.3 & 16.0 & 85.0 \\
\hline Power & 86.1 & 86.1 & 94.9 & 94.9 & 94.9 \\
\hline DMS & 107.3 & 107.3 & 133.1 & 133.1 & 133.1 \\
\hline AOCS & 178.6 & 178.6 & 190.7 & 171.3 & 190.7 \\
\hline CPS & 1.8 & 1.8 & 3.5 & 3.5 & 3.5 \\
\hline Communications & 158.0 & 158.0 & 240.1 & 158.0 & 33.4 \\
\hline Harness & 11.5 & 11.1 & 17.1 & 11.5 & 12.4 \\
\hline $10 \%$ System Margin & 58.9 & 56.6 & 87.4 & 58.8 & 55.3 \\
\hline Heaters & 487.0 & 341.0 & 177.0 & 22.0 & 22.0 \\
\hline Total(MPO) & 1134.5 & 963.6 & 1137.9 & 669.1 & 630.1 \\
\hline $\operatorname{Mio}^{f}$ & 300.0 & 300.0 & & & \\
\hline Total(Mio) & 300.0 & 300.0 & & & \\
\hline \multicolumn{6}{|l|}{ MTM } \\
\hline Power & 74.1 & 74.1 & & & \\
\hline DMS & 26.9 & 22.0 & & & \\
\hline MEPS & 25.1 & 31.8 & & & \\
\hline CPS & 19.5 & 3.2 & & & \\
\hline Harness & 2.9 & 2.6 & & & \\
\hline $10 \%$ System Margin & 14.9 & 13.4 & & & \\
\hline SEPS Thrust Power & 0.0 & 10291.0 & & & \\
\hline Heaters & 1707.0 & 186.0 & & & \\
\hline Total (MTM) & 1870.4 & 10624.0 & & & \\
\hline Total & 3304.8 & 11887.7 & 1137.9 & 669.1 & 630.1 \\
\hline $\begin{array}{l}\text { Power available at spacecraft } \\
\text { level from solar arrays }\end{array}$ & 6393.8 & 12993.7 & 1633.6 & 665.3 & \\
\hline Power margin & $48 \%$ & $9 \%$ & $30 \%$ & $3 \% \mathrm{~g}$ & \\
\hline
\end{tabular}

${ }^{\mathrm{a}}$ Example at $1.19 \mathrm{AU}$ from the Sun

${ }^{\mathrm{b}}$ Example at $0.61 \mathrm{AU}$ from the Sun, 2 SEPS thrusters at $290 \mathrm{mN}$ thrust level each

${ }^{c}$ Example at Mercury True Anomaly $90^{\circ}$, full payload, X/Ka-band Tx

${ }^{d}$ No payload operations, no Ka-band Tx (8 hours/day)

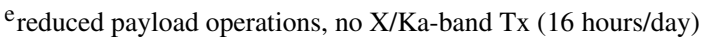

${ }^{\mathrm{f}}$ Forfeitary allocation to Mio for periodic check-out and/or heaters

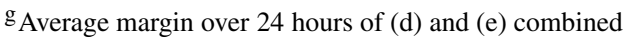




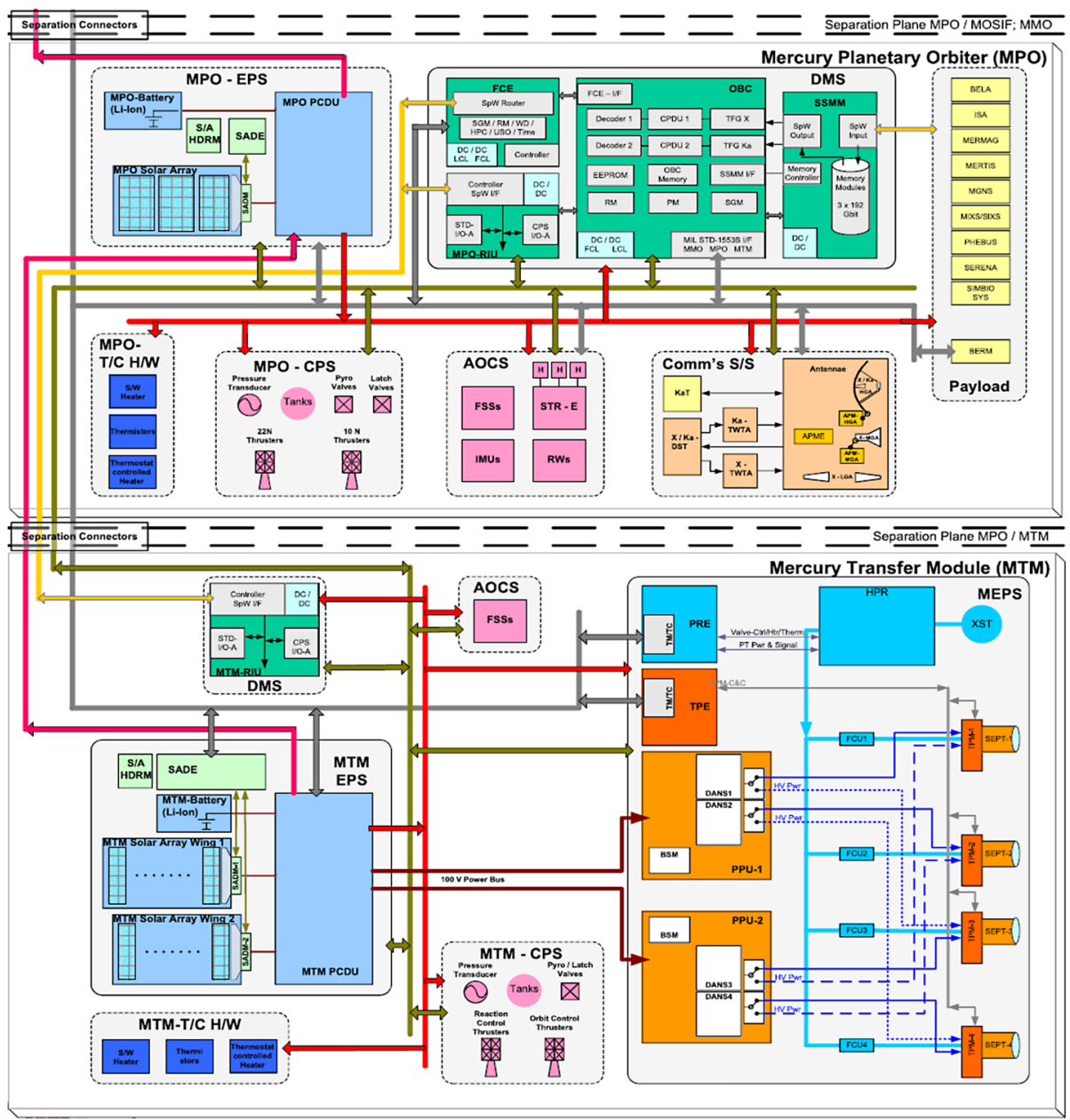

Fig. 7 MPO and MTM Block Diagram. Abbreviations: APME Antenna Pointing Mechanism Electronics, APR Array Power Regulator, AOCS Attitude and Orbit Control System, CPS Chemical Propulsion Subsystem, DST Deep Space Transponder, DANS Discharge Anode and Neutralizer Supply, EPC Electronic Power Converter, FCU Flow Control Unit, HGA High Gain Antenna, HPR High Pressure Regulator, IMU Inertial Measurement Unit, LGA Low Gain Antenna, LPT Low Pressure Transducer, MGA Medium Gain Antenna, MMO Mercury Magnetospheric Orbiter Mio, MOP Mercury Planetary Orbiter, MTM Mercury Transport Module, MOSIF MMO sunshield and interface Structure, MEPS MTM Electric Propulsion System, OBC OnBoard Computer, Pwr Power, PCDU Power Control and Distribution Unit, PPU Power Processing Unit, PRE Pressure Regulation Electronics, PT Pressure Transducer, PPS Pulse Per Second, RMU Rate Measurement Unit, RIU Remote Interface Unit, RT Remote Terminal, S/W Software, SADE Solar Array Drive Mechanism Electronics, SEPT Solar Electric Propulsion Thruster, SSMM Solid State Mass memory, SpW SpaceWire, STD Standard, SOC State of Charge, TMTC Telemetry/Telecommand, TCS Thermal Control Subsystem, TPE Thruster Pointing Electronics, TWT Travelling Wave Tube; image provided by Airbus Defence and Space $\mathrm{GmbH}$

The sunshield is a metal truss structure covered with MLI with appropriate thermal finishes inside and outside to ensure suitable temperatures for Mio. The conical shape of the MOSIF - with an opening angle of about $16^{\circ}$ - is needed to allow for the lateral velocity and wobble of the Mio generated during its spin-up at separation. 
Fig. 8 BepiColombo MTM

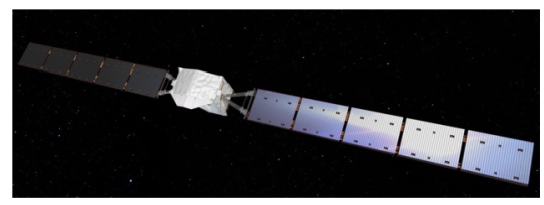

Fig. 9 BepiColombo sunshield MOSIF during vibration test in October 2015

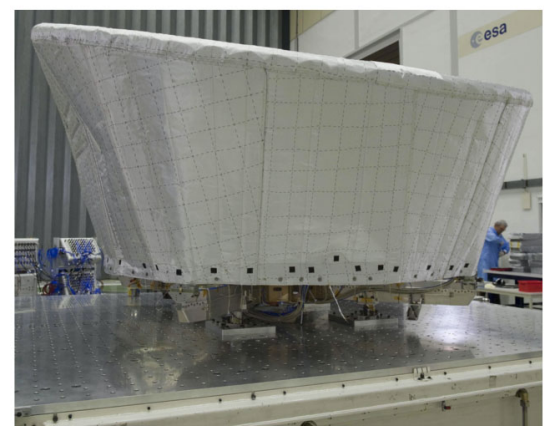

Fig. 10 BepiColombo cruise configuration

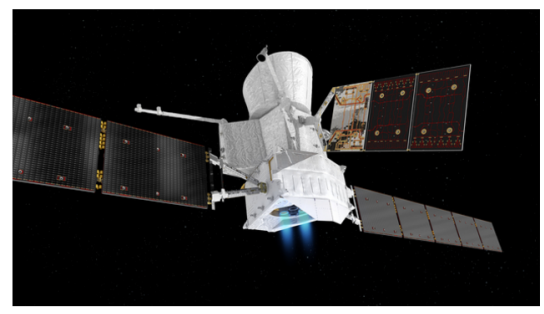

Fig. 11 BepiColombo in its stacked launch configuration with solar arrays folded during its acoustic test in mid 2017

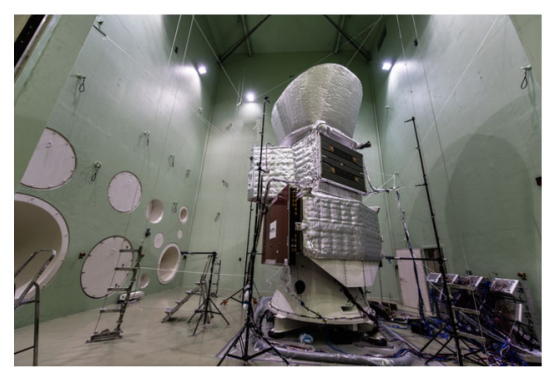

\subsection{Mission Scenario}

BepiColombo is unique in the way that it comprises three complete spacecraft flying together as a mechanically and electrically coupled stack (see Figs. 10 and 11) from launch up until the Mercury orbit insertions of its two scientific orbiters, Mio and MPO. The design of the mission requires that the two orbiters are brought to Mercury by the Mercury Transfer Module (MTM). The high delta- $\mathrm{V}$ required to put a spacecraft into a Mercury orbit and the total mass to be carried pose challenging requirements to the mission analysis and to the propulsion systems. In order to bring two spacecraft from Earth to Mercury, BepiColombo uses a hybrid approach in which gravity assist maneuvers at Earth, Venus, and Mercury are 
employed together with a Solar Electric Propulsion (SEP) system. Planetary gravity assist maneuvers are very effective in changing the spacecraft velocity with respect to the Sun. However, they require a longer interplanetary transfer duration once a sequence of swingby's has been selected. The advantage of such trajectories, thanks to electric propulsion, is that they offer large flexibility to design the trajectory within the boundaries of launcher capability, spacecraft mass, spacecraft availability, and flight duration at very low propellant cost (Langevin 2000).

A total of nine planetary gravity assists will be performed by the BepiColombo spacecraft during a 7.2-years interplanetary cruise as shown in Fig. 12 with a total active SEP time of around 650 days ( $25 \%$ of the total Cruise duration), resulting in more than $3 \mathrm{~km} / \mathrm{s}$ delta-V imparted to the spacecraft. For the majority of their active life, the thrusters will operate close to their maximum thrust level; thrust arcs at lower levels are concentrated in the first interplanetary phase where the distance from the Sun is greater and the limiting factor is the available power from the solar arrays. The first flyby occurred at the Earth on 10 April 2020, followed by two encounters at Venus (Oct 2020 and August 2021) and six at Mercury until orbit insertion the end of 2025.

In order to achieve this optimal sequence, the spacecraft is required to perform SEP thrust arcs between most planetary encounters. Each of the four SEP engines can provide between $75 \mathrm{mN}$ and $125 \mathrm{mN}$ depending on the Sun distance; up to two engines can be fired simultaneously. With an initial spacecraft mass of around 4 tons, the thrust-to-mass ratio at the beginning of cruise phase is approximately $4 \times 10^{-5} \mathrm{~N} / \mathrm{kg}$, while the specific impulse is close to $3800 \mathrm{~s}$. Since very precise navigation is necessary for the planetary gravity assist maneuvers (which cannot be done with SEP) the BepiColombo MTM contains also a set of chemical propulsion thrusters (Gray et al. 2019). The arrival conditions at Mercury are selected so that a "weak" gravitational capture is possible: this technique takes advantage of the gravitation pull of both the Sun and Mercury to achieve a temporarily closed orbit around the latter. The most demanding requirement needed to implement this technique is to have a sufficiently low arrival velocity at Mercury. This was also one of the primary objectives for implementing SEP on BepiColombo, since gravity assists cannot be used for this purpose. The major advantage of adopting the "weak" stability boundary gravitation capture technique is the added robustness of having multiple attempts available for the first crucial manoeuvre in the Mercury Orbit Insertion (MOI) sequence.

Due to thermal control challenges, and to the need to fly three spacecraft together as one physical stack, one needs to consider a number of interactions and constraints. The cruise mission cannot simply be flown with the stacked spacecraft in a single, sun-pointing orientation. Deviations to the purely Sun oriented attitude originate from the need to thrust in an optimised direction, while also performing reaction wheel off-loading during the use of the solar electric propulsion thrusters. Prior to each planetary fly-by, it is necessary to have sufficient degrees of freedom in the range of Sun angles permitted on the stack, in order that the Attitude and Orbit Control System (AOCS) can perform any fine trajectory correction manoeuvres. In addition, during these critical times, any extra degree of flexibility in the spacecraft attitude is helpful for optimising the orientation of antennas and thereby increasing the communication opportunities back to Earth.

When the spacecraft reaches the inner part of the Solar System, the solar flux is not the limiting factor and the solar arrays are tilted with respect to the Sun direction to avoid excessive temperatures, while still providing all the necessary power required by the thrusters at maximum thrust level. After some initial check-outs and tests during the post-launch Near Earth Commissioning Phase, the BepiColombo SEP engines have successfully completed its first two thrust arcs (each about 2 month long) in preparation for the spacecraft's 
Fig. 12 The trajectory of BepiColombo from Launch on $20^{\text {th }}$ October 2018, over the 7.2-year cruise until Mercury orbit capture in December 2025. The mission trajectory includes one Earth flyby, two consecutive Venus flybys (300 days apart), which reduce the perihelion to nearly that of Mercury. A sequence of 6 Mercury flybys passing through $3: 2,4: 3,5: 4$ and $1: 1$ resonances with Mercury's orbit period and a $180^{\circ}$ singular transfer lowers the spacecraft velocity relative to the planet to $1.85 \mathrm{~km} / \mathrm{s}$. Five final thrust arcs further reduce the relative velocity such that the spacecraft will be weakly captured by Mercury's gravity on 5 December 2025 even if no orbit insertion maneuver takes place

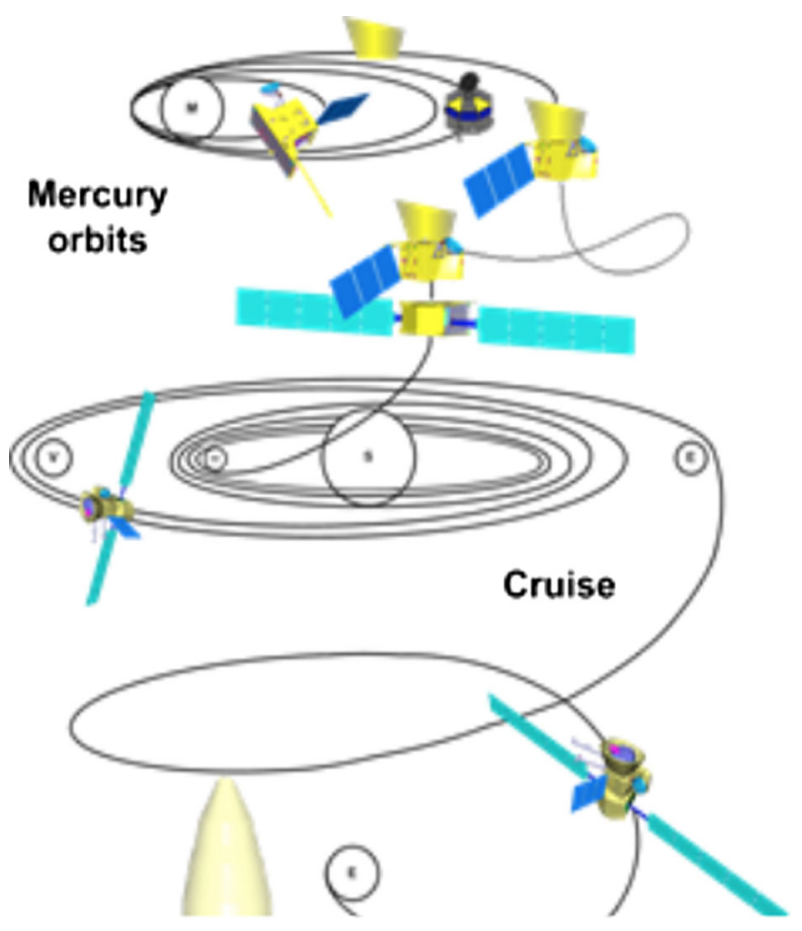

first planetary encounter with the Earth. This flyby was successfully performed on 10 April 2020 .

\subsection{BepiColombo Ground Segment}

The BepiColombo Ground Segment is quite complex. ESA's Mission Operations Center is responsible for mission operations of the composite stack during the interplanetary transfer and of the MPO once at Mercury. The heart of the Mission Operations Center is the Mission Control System used for telemetry processing and commanding. The Flight Dynamics System covers the functionality of spacecraft navigation. Special development was needed to enable autonomous re-configuration to support solar-electric, low-thrust navigation. A Mission Planning System is used for the preparation of commanding products to the spacecraft and ground segment.

JAXA is responsible for Mio operations throughout the mission. Mio operations take place at Sagamihara Space Operation Center (SSOC)/JAXA. Mio specific functions have been implemented such as an special interface to receive data from the ESA mission operations center data archive. A subset of the mission control system has been deployed at ESA for Mio cruise operation support and also serves as a backup in case of problems. Direct interaction between the JAXA Control Center and Mio will happen after Mio's separation in late 2025.

The BepiColombo Science Ground Segment of ESA consists of the Science Operations Control System and the Archive Systems (scientific and operational). The main functions are Science Planning, Science Data processing, Quick look Analysis, and Archiving. For MPO, the first-level data processing is performed after the telemetry is received on-ground, 
typically within hours. Calibrated and higher-level products are generated using instrumentspecific calibration and reduction processing pipelines. Generated science products will be compatible with the new version of NASA's Planetary Data System (PDS) standards, PDS4 and can be immediately archived.

ESA's Deep Space $35 \mathrm{~m}$ ground stations at New Norcia (Australia), Cebreros (Madrid, Spain) and Malargüe (Argentina) are used to operate the BepiColombo mission. The sites have been selected to minimise radio-frequency interferences and provide near-continuous contact to interplanetary probes. All antennae support X-band up- and downlink. Ka-band downlink is deployed at Cebreros and Malargüe, while Ka-band uplink is only available at Malargüe. On the JAXA side the Deep Space Antenna at Usuda is currently envisaged to operate Mio after the separation at the end of 2025. However, the new Misasa Deep Space Station of JAXA will become operational in April 2021 for future use on BepiColombo. If needed, a cross-support agreement between ESA and JAXA allows ESA to use the JAXA deep space antennas also during cruise. The BepiColombo mission baseline also includes the use of the DSS-25 antenna at Goldstone, part of NASA's Deep Space Network (DSN), for the BepiColombo radio-science experiment. In summary, the BepiColombo Ground Segment has to deal with specific operational challenges such as electric propulsion, two-spacecraft operations, and a very complex Mercury Orbit Insertion sequence to deliver MPO and Mio into their respective science orbits. In addition, the mission also requires the adoption of specific measures for knowledge preservation and long-term maintenance. ESA's Planetary Science Archive will host all the MPO and Mio science data, as well as any relevant spacecraft and instrument housekeeping information and be used to distribute the data to the scientific community. Following the six-month proprietary period, and after feedback from the Instrument Teams has been received, raw and calibrated data available in the archive are routinely released to the public. The Mio science data will be also available in JAXA's Data ARchives and Transmission System (DARTS). For more details on the BepiColombo Ground Segment see Montagnon et al. (2021).

\section{Science Objectives}

The aim of BepiColombo is to take high-resolution (i.e., spatial and temporal) observations and make many new observations of the entire surface and within the environment of Mercury. Compared with MESSENGER, the southern hemisphere will be observed from much lower altitudes, and the two orbiters will perform simultaneous measurements of the magnetic field at different positions. In addition, they will observe interactions between the planet and the solar wind (space weather). Both BepiColombo Orbiters carry instruments not included in the MESSENGER payload, such as a thermal infrared spectrometer, a full complement of plasma physics instrumentation, and a triple-band, radio-science instrument with an on-board accelerometer. The BepiColombo mission will contribute to answer many of the new questions revealed by the discoveries of MESSENGER.

Decades of scientific investigations of Mercury from the first Earth based observations to the last in-orbit observations of MESSENGER have revealed the complexity of Mercury and the important interactions between its interior, surface, magnetosphere, and exosphere. Deciphering the surface composition of Mercury, together with a better understanding of its interior structure and evolution will certainly provide new insights into the formation of planets. There is a fast-growing list of exoplanets with orbits close to its central stars like Mercury. However, up to date Mercury-sized planets have not yet been detected around other stars. Characterizing the magnetic field properties and its magnetosphere with respect 
to the dynamic solar environment and its implication in the modification and preservation of the Hermean exosphere will explain the role and importance of Mercury's core dynamo. Mercury has the most compressed magnetosphere due to the small dipole field and the short distance to the Sun allowing the solar wind interact directly with the planetary surface. The description of Mercury's geological history mostly expressed through its volcanism, tectonism, and impact cratering will help to reconstruct Mercury's geologic record. Determining the volatile budget of Mercury, its nature and evolution through time is a critical aspect for understanding the source and distribution of volatiles in the Solar System. All of those important scientific investigations will be addressed by BepiColombo, and will contribute to the improved knowledge of the Solar System. The main scientific objectives of the BepiColombo mission are:

- Investigation of the origin and evolution of a planet close to its parent star

- Investigation of Mercury's figure, interior structure, and composition

- Investigation of the interior dynamics and origin of its magnetic field

- Investigation of the exogenic and endogenic surface modifications such as cratering, tectonics, and volcanism

- Investigation of the composition, origin, and dynamics of Mercury's exosphere and polar deposits

- Investigation of the structure and dynamics of Mercury's magnetosphere

- Test of Einstein's theory of general relativity

These science objectives of BepiColombo can be related to three major topical areas that are highly correlated with Mercury's envelopes, namely:

- Internal structure and physical characteristics (Interior)

- Surface composition and evolution (Surface)

- Hermean dynamical environment (Magnetosphere and Exosphere)

These topics are by no means isolated but strongly coupled to each other and essential in the understanding of Mercury's global picture.

\subsection{Interior}

Understanding the interior structure of terrestrial planets and satellites is pivotal to understand the physics and chemistry of these bodies. The dynamics of the surface with respect to volcanic and tectonic activity depend on the thickness of the crust and lithosphere, as well as the characteristic of the silicate mantle and the chemistry of the planet.

Mercury's core can be constrained through indirect measurements only. Geophysical, magnetic, and geochemical observations have led to a core size estimate of about $2000 \mathrm{~km}$ in radius, with an uncompressed density of about $7000 \mathrm{~kg} / \mathrm{m}^{3}$, and an $\mathrm{Fe}-\mathrm{Ni}$ dominant composition with light elements such as $S i$ and $S$ (Margot et al. 2018). Precise determination of the core's state, size, and composition is critical for nearly all science investigations on Mercury (i.e., surface composition, magnetic field, etc..). Although the accuracy on the determination of the core's parameters has been significantly improved (Genova et al. 2019), there are still large uncertainties that prevent an accurate determination of the inner core radius. Given that Mercury is occupying a Cassini state (i.e., state no1), the interior structure can be reliably determined through accurate measurements of the gravity field, the topography, the libration amplitude, and the tides of the planet. BepiColombo seeks to provide unprecedentedly accurate measurements of the Love Numbers, $h 2$ (tidal amplitudes) and $k 2$ 
(tidal potential), the principal Moment of Inertia, and the Libration Amplitude of Mercury through dedicated-instruments and joint-instruments observations.

Mercury's crustal thickness is usually accepted to be on the order of $50 \mathrm{~km}$, the outer silicate shell thickness being about $400 \mathrm{~km}$. Nonetheless, the uncertainties are relatively large, and the crust could be much smaller. For instance, much of the knowledge of Mercury's crust is derived from measurements which are incomplete or not sufficiently resolved in particular for the surface in the southern hemisphere. The crustal thickness has very important implications in the formation of Mercury's crust and the material currently exposed on the surface, thus accurate measurements of its thickness are important for scientific investigations. BepiColombo seeks to significantly improve the estimates of the crustal thickness through observations done with a suite of dedicated instruments (radio science, altimetry, high resolution imaging) and by equally covering both hemispheres with similar viewing conditions. Also, the determination of the distribution of mascons and other gravity anomalies in the south must await BepiColombo arriving in its close polar orbit with the shift of its periapsis over the course of the mission. Tracking of the MPO spacecraft will help refining the gravity field of Mercury and will yield refinements to the size and physical state of its core to obtain additional constraints on models of the planet's internal structure and test theories of gravity with unprecedented accuracy (Genova et al. 2021).

\subsubsection{Fundamental Physics}

Mercury is the innermost and fastest planet of the solar system. Relativistic effects on its motion are therefore larger than for any other solar system body, making it a unique laboratory for probing gravity and its theoretical description. The brilliant explanation of the anomalous periastron advance of Mercury's orbit, incompatible with Newtonian physics, was the first experimental success of general relativity. BepiColombo can repeat classical tests with much improved accuracy and, thanks to the proposed system configuration, explore new aspects of gravitational theories (Iess et al. 2021).

Mercury is such a unique body for gravitational physics and the most affected by relativistic corrections. Precise measurements of radio signal frequency and propagation time provide a determination of post-Newtonian parameters. The parameterised post-Newtonian (PPN) expansion is a very useful way to classify theories of gravity in the weak field, low velocity approximation pertinent to Solar System dynamics (De Marchi and Cascioli 2020). The focus of past classical tests of general relativity has been on the Eddington parameters $\beta$ and $\gamma$ controlling respectively the non-linearity in the superposition of gravity fields and the space curvature produced by a unit mass. Those effects are strongly magnified when the signal passes close to the Sun, i.e. the solar superior conjunctions. BepiColombo seeks to determine other parameters of the parameterised post-Newtonian formalism, most notably $\alpha 1$ and $\alpha 2$, related to the existence of preferred frames, and $h$, related to violations of the strong equivalence principle through precise radiometric measurements of the BepiColombo spacecraft.

In spite of striking experimental success, questions about the range of validity of general relativity come not only from the derivation of the field equations, based upon an attractive but arbitrary criterion of mathematical beauty and simplicity, but also from the impossibility to reconcile general relativity with quantum mechanics. In addition, the cosmological evidence for dark matter and dark energy may well point to violations of the Newton-Einstein theory of gravity. The increasing accuracy of space experiments makes it possible to evaluate the violations of Einstein's theory (Voisin et al. 2020; Archibald et al. 2018). In recent theoretical developments a new paradigm has recently emerged. The equivalence principle 
and general relativity are generically and jointly violated due to a small scalar field with its own dependence on time and space, which is expected to affect all the different physical interactions. BepiColombo seeks to test the compliance of the gravitational contributions of the Earth, the Sun, and Mercury with the equivalence principle, to see whether they fall within the same acceleration in the gravitational field of a fourth massive body. For more details see Genova et al. (2021).

\subsection{Surface}

Mercury is an end-member and the planet closest to the Sun. The reason for which this planet ended up with its of size, uncompressed density, and composition are still mysterious. It occurs in a close-to-star region analogous to those in which rocky exoplanets have been discovered. Mercury can shed light on how Earth and other rocky planets formed and evolved and on how rocky exoplanets formed.

Mercury is extremely diverse in terms of the composition of its surface (Peplowski et al. 2015; Weider et al. 2015; Vander Kaaden et al. 2017). Observations from MESSENGER have revealed numerous puzzles about Mercury's composition and its place in the Solar System formation. The unexpected increased concentrations in elements such as $K, M g$, and $\mathrm{Si}$ (Nittler et al. 2018) provide constrains on the formation and evolution of Mercury. The fact that the spatial distribution of $M g$, for instance, is distinct from that of other chemical markers suggests different initial compositions and/or magma genesis. The oldest crust of Mercury is thought to be represented by the low reflecting material (LRM), terrains potentially enriched in carbon, in its graphite form (Peplowski et al. 2016). Elemental composition mapping from MESSENGER was largely limited to the northern hemisphere, and most maps are at low (hundreds of kilometers) spatial resolution because of limited count rates in the measuring instruments and the eccentricity of MESSENGER's orbit. The paucity of iron at Mercury's surface (in its reduced phase) is a particular open question that needs to be resolved so as to better model the early history of Mercury (Izenberg et al. 2014). BepiColombo seeks to place constraints on theories on the origin and formation of Mercury by determining the mineralogical and elemental composition of Mercury's oldest and diverse terrains, including the southern hemisphere.

The spin/orbital resonance of the orbit and its high eccentricity leads to a very unusual phenomenon of the planet: the so-called "hot meridians". There are two meridian belts on the surface which regularly get the largest flux of solar radiation at the pericenter of every other orbit. The surface at the equator around the "hot meridians" has the largest variation of the temperature from $700 \mathrm{~K}$ at midday down to $100 \mathrm{~K}$ at night. Volatile evaporation from the surface might be ongoing on Mercury. If this is the case, an annual variation of abundances of volatiles would be expected for areas located near the "hot meridians" and the equator. These variations, provided they could be detected, would allow one to build a dynamic model of evaporation and condensation of volatiles at Mercury at the present time.

Unexpectedly high concentrations of volatiles have been observed with respect to that of refractory elements and apart from elemental composition, there are several examples of volatile influence on Mercury. Mercury's polar water ice deposits (Delitsky et al. 2017), putative pyroclastic deposits from explosive volcanism, and specific land-forms, so-called hollows are features that exhibit the complexity of the distribution of volatiles on Mercury (Blewett et al. 2013). Given the limitations of previous observations on Mercury, the volume and distribution of volatiles could be significantly underestimated. For instance, the distribution of polar ice deposits is largely unknown in the southern hemisphere (Chabot et al. 2018). Yet, those volatile-rich features are absolutely fundamental in understanding Mercury's history. Are the volatiles preserved during Mercury's formation (Killen et al. 1997) and if so, 
how, or are the volatiles delivered to Mercury at a later phase? Those questions have massive implications for the understanding of volatiles throughout the Solar System (Lawrence 2017). BepiColombo seeks to improve the characterisation of the volatile distribution and its quantification through global mapping of all volatile-rich features on the surface, while using the relatively homogeneous coverage and high count rates of its dedicated instruments due to the low eccentricity MPO orbit as compared to that of MESSENGER.

Hollows are landforms not seen on other planetary bodies (Blewett et al. 2011). The scientific consensus of hollows being the manifestation of degassing from the subsurface due to the high volatile content of the planet is supported by various remote sensing observations and preliminary laboratory analyses. Nonetheless, key questions are still unresolved about those new planetary features such as the process driving the volatile loss and the mechanism/timescale by which the hollows grow, the identity of the volatiles that are lost during the process, and the origin of those volatiles. Hollows are certainly playing a key role in the most recent changes visible on the surface of Mercury, and their associations with specific terrains such as the LRM could provide critical information on Mercury's history (Blewett et al. 2016). BepiColombo seeks to characterise hollows' properties with unprecedented spatial and spectral resolution, to complete the spatial distribution by mapping the southern hemisphere, and to provide new insights into Mercury's volatile budget and most recent geological activities.

Mercury's tectonic history is dominated by crustal shortening features indicative of a global contraction of the planet during the Calorian period (i.e., 3.9-3.3 Ga, Byrne et al. 2014, Marchi et al. 2013). Extensional deformation and long-wavelength undulation of the topography highlight the complex changes in activity that Mercury has undergone together with extrusive volcanism (Head et al. 2008) on most of its surface. Some observations point to tectonic and volcanic activity that happened very recently (on geological time scales). Recent activity is not necessarily surprising given that Mercury still has a very active interior and secular cooling of its core. The combination of all geological features on the surface tell us about Mercury's history, but this history still has uncertainties and unknowns that are severely limiting our understanding of Mercury history. Many of those uncertainties rely on identifying the most ancient geological features which will help us to characterise the initial state of Mercury. BepiColombo seeks to provide scientific observations that will characterise the oldest geological features and thus define better starting points to understand Mercury's evolution.

The nature of the high-magnesium region, mechanisms of source processes for neutral and ionized species in the exosphere and magnetosphere, and details of the space weathering of Mercury's surface are also open issues (Domingue et al. 2014), as are the identification and roles of various minor exospheric species. For more details see Rothery et al. (2020).

\subsection{Magnetosphere and Exosphere}

Characterizing the environment around Mercury is of primary importance to derive the structure of Mercury's internally generated magnetic field and to understand the dynamics and structure of the interaction between the planet and the Sun (Milillo et al. 2020, this journal). The interaction of Mercury with the external medium, i.e. micro-meteoroids, radiation, and solar wind contributes to the definition of the dynamics and the structure of Mercury's magnetosphere and exosphere (space weather) and, over long time intervals, also to the modification of the surface (space weathering). The close proximity to the Sun makes many effects more intense than those on other objects in the Solar System. 
Within the Solar System out of the planetary bodies only Mercury, Earth, and Ganymede have intrinsic magnetospheres, e.g. produced by an internal, dynamo-driven, planetary magnetic field. Mercury's magnetosphere is less intense than its Earth counterpart, and it is subject to an order of magnitude higher solar wind fluxes (i.e.: Slavin and Holzer 1981). In nominal conditions, the surface is partially shielded by the magnetosphere, but the re-connection rate is generally high, allowing plasma entry inside the magnetosphere (Di Braccio et al. 2013). In extreme conditions, the dayside magnetopause could be completely eroded, leaving Mercury's surface unprotected (Slavin et al. 2014). The results from MESSENGER revealed a remarkably dynamic and active Mercury's magnetosphere populated by ions of the solar wind as well as of planetary origin (Raines et al. 2015) with substantial acceleration and precipitation of energetic particles to the surface (Slavin et al. 2018). These results make it even more imperative to measure the thermal electron population, plasma convection dynamics, and electromagnetic wave environment. The understanding of the magnetosphere's structure and dynamics at a close distance to the Sun is a major goal of BepiColombo. Moreover, the remarkable dynamics of the magnetosphere and the proximity of the planet core to the surface raise fundamental questions regarding the strong link between the internal and the interplanetary magnetic fields (Jia et al. 2015), a topic that will be best addressed with the combined two-point magnetic field observations that are planned with BepiColombo's MPO and Mio orbiters.

The exosphere, i.e. the collision-less neutral gas envelope surrounding Mercury, is generated by the surface release processes driven by external sources, like sun radiation, impacting ions, and micrometeoroids (Killen et al. 2007), and possible transient sources, like volatile activity. These surface release processes are extremely influenced by the magnetosphere and by the surface characteristics. The exosphere of Mercury, with its exposure to intense solar wind flows and a high dust flux environment, is a key laboratory for investigating generation, transport and loss processes of a collision-less atmosphere. In particular, MESSENGER was not instrumented to survey the dust environment of Mercury, although the altitude distribution and temporal variation of the density of neutral atoms in the exosphere point to micrometeoroid and comet stream impacts as an important source process (Killen and Hahn 2015). Hence, direct measurements of the dust environment planned on BepiColombo should confirm these inferences of the dynamic sources for the exosphere. With a dedicated orbiter to probe the environment around Mercury, Mio, and instruments on the planetary orbiter, MPO, BepiColombo seeks at better determination of the structure and variability of the environment in term of external drivers and planetary response. The discovery of hollows on the Mercury surface (Blewett 2018) and the confirmation of water-ice deposits in the polar regions by MESSENGER (Chabot et al. 2016) suggest that sensitive neutral and ion mass spectrometers might be able to probe material released from these regions. The high performances of instruments carried by BepiColombo may allow determination of detailed characterisations of the material from the surface and specifically above the hollows and the polar deposits.

During its history, Mercury's surface suffered intense bombardment of micrometeoroids and ions coupled to a strong radiation flux from the sun, thus the space weathering altered its physical and chemical properties substantially. The individual contribution of each weathering process is difficult to evaluate given the complex interactions between the surface and the environment also depending on the elemental composition, volatile content, and mineralogy. The understanding of space weathering on Mercury may have also implications for understanding the surfaces of other planets. Therefore, BepiColombo seeks to constrain better the individual processes that weather Mercury's surface through dedicated in-situ measurements and it provides a comprehensive view of the weathering processes also in action 
Fig. 13 Earth captured by one on the monitoring cameras (Cam-2) shortly before closest approach on 10 April 2020

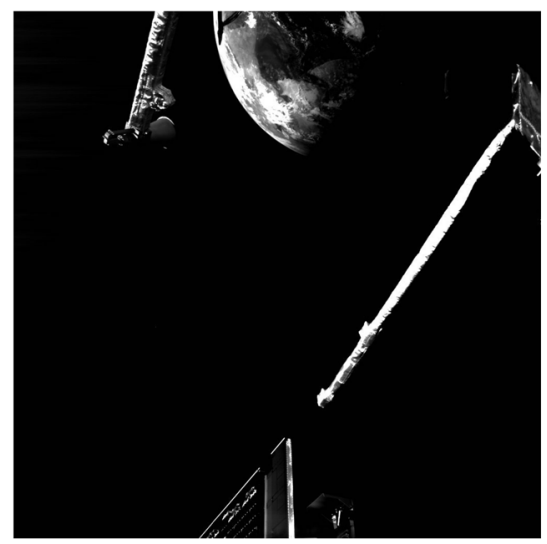

Fig. 14 Venus captured by one on the monitoring cameras (Cam-2) during closest approach on 15 October 2020

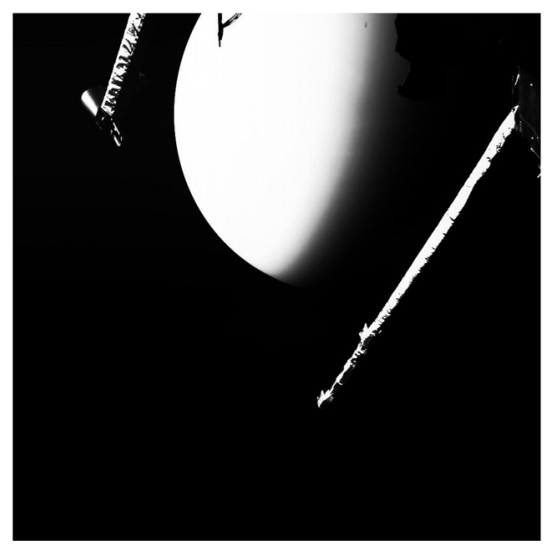

elsewhere in the Solar System. Finally, Mercury, being an end member, is a key object for the scientific community to understand how the environments of planets are modified by energetic phenomena. It is a laboratory for space weather and space plasma physics, for surface-bounded exospheres, which are seen throughout the solar system (e.g., the Moon, Galilean satellites of Jupiter) and for space weathering processes. The achievement of the major scientific goals mentioned previously will provide unique inputs regarding the exospheric and the magnetospheric dynamics and will constitute a test bench for space weather and space weathering processes on silicate-dominated solar system bodies. For more details see Milillo et al. (2020).

\subsection{Science During Cruise and Flybys}

In addition to the biannual instrument check-outs that are scheduled to monitor the health and functional performance of the instruments, the only initially planned science measurements performed during the cruise phase are those to support the General Relativity (GR) investigation of MORE during dedicated solar conjunctions (Imperi and Iess 2017).

Lately, several other opportunities have also been identified to acquire scientifically useful data from both MPO and Mio instruments during cruise. However, such opportunities 

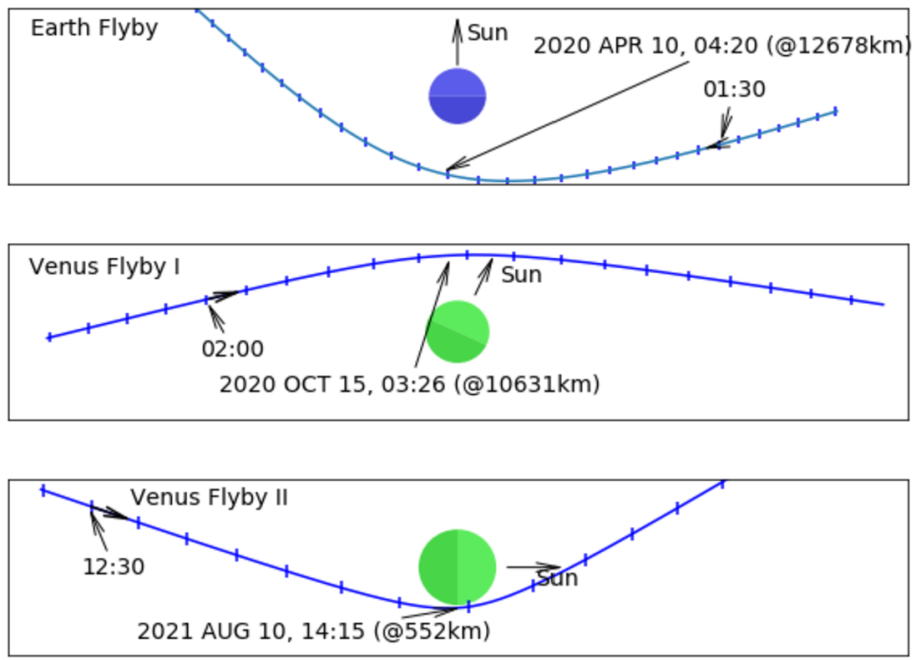

Fig. 15 BepiColombo Earth and Venus flybys during the cruise phase. All sketches of the flybys are in a polar view indicating the illumination conditions at the estimated time and position of the closest approach as well as the flyby direction

are subject to short-term feasibility assessments with respect to spacecraft design and operations constraints. The related cases cover science observations during the flybys, solar wind observations, solar corona observations, and dust particle measurements during possible cometary dust trail crossings.

The GR observations during solar superior conjunctions will allow the derivation of the parameterized formulation of the Post-Newtonian expansion, especially the Eddington and the Nordtvedt parameters (Di Stefano et al. 2021). The determination of these parameters will allow to study the possible violations of the strong equivalence principle. The first six solar conjunctions observations are currently planned for this experiment: 11 March 2021 (14.9 days), 29 January 2022 (12.7 days), 10 July 2023 (8.5 days), 28 January 2023 (13.8 days), 26 June 2023 ( 7.7 days), and 30 November 2023 (31.2 days). The data of the GR experiment obtained during the solar superior conjunctions also allow the determination of the total electron content of the solar corona.

During the cruise phase, several in-situ instruments can monitor the solar wind. Together with data from, e.g., ESA's Solar Orbiter mission (Müller et al. 2013) and NASA's Solar Parker Probe mission (Fox et al. 2016), two missions also in operation at solar distances less than $1 \mathrm{AU}$, multi-point measurements of the solar wind within the inner Solar System can be achieved, and, if caught, events like coronal mass ejections (CMEs), interplanetary CMEs (ICMEs), stream interaction regions (SIRs), co-rotating interaction regions (CIRs), magnetic holes, and other features within the solar wind could be observed. Monitoring of the plasma environment around the spacecraft will, in addition, provide valuable engineering and scientific information. The cosmic gamma-rays will be continuously monitored during the cruise except for periods of operating the electrical ion propulsion system. Cosmic gamma-ray bursts (GRBs) will be recorded, which are thought to be produced by the enigmatic cosmological explosions of stars like supernovae or to originate from the merger of binary neutron stars (Hurley et al. 2017). It is necessary to find the direction of incoming burst gamma-rays to locate its source in the sky. From the data from BepiColombo at the interplanetary space one will provide the local time of detection of an GRB, which could 

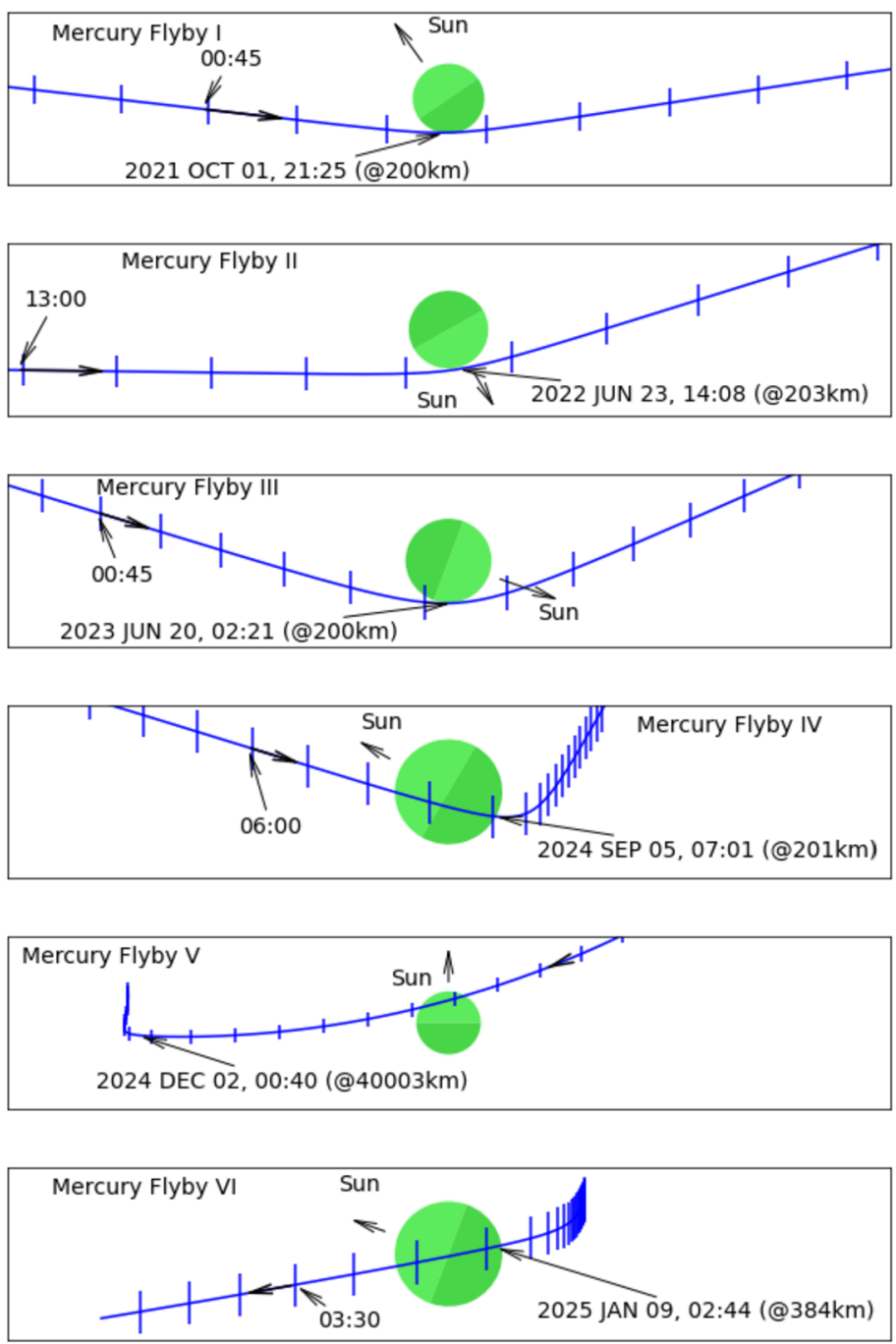

Fig. 16 BepiColombo Mercury flybys. Details are likely to change due to upcoming manoeuvre changes. All sketches of the flybys are in a polar view onto Mercury indicating the illumination conditions at the time of the closest approach as well as the flyby direction

be used in the world-wide program of interplanetary multi-mission network for determining the sky position of the outburst.

The planetary flybys will provide unique chances for operating several instruments and obtain data in support of scientific analysis and instrument calibration. On April 10, 2020, BepiColombo passed the Earth at $04: 24: 58$ UTC at a distance of $12693 \mathrm{~km}$ (Fig. 13). The distance approach to the Moon (i.e., $\geq 300.000 \mathrm{~km}$ ) provided opportunities for remote sensing instruments. Unfortunately, the high-resolution and stereo cameras and also the VIS and near IP spectrometer, three major remote sensing instruments of BepiColombo have a blocked field of view by the MTM until its arrival at Mercury, hence it could not observe the Earth or Moon. The geometry of the flyby provided favorable conditions for exploring 
spacecraft orbit determination of ingress and egress arcs, which are often mentioned as the "Earth flyby anomaly" (Anderson et al. 2008). Measurements performed during the Earth flyby of BepiColombo showed no sign for the Earth flyby anomaly. Ingress and egress data arcs have fitted without any problem.

A first Venus flyby was performed on October 15, 2020. BepiColombo passed Venus at $03: 58: 31$ UTC at a distance of $10721,6 \mathrm{~km}$ and the velocity change was about $-3.25 \mathrm{kms}^{-1}$ (Fig. 14). Seven of the eleven science instruments onboard MPO, plus its radiation monitor, and three of five instruments onboard Mio were active during the flyby. While the suite of sensors are designed to study the rocky, atmosphere-free environment at Mercury, the flyby offered a unique opportunity to collect valuable science data at Venus. Scientists hope to be able to provide some atmosphere temperature and density profiles, information about the chemical composition and cloud cover, and on the magnetic environment interaction between the Sun and Venus. A second Venus flybys will follow on August 11,2021 . The two Venus flyby geometries differ significantly in both closest approach distances (10721 and $551 \mathrm{~km}$ respectively, see Fig. 15), and regions of the magnetosphere that will be sampled. Although investigations focusing on the surface and interior of the planet will not be possible given the rapid flyby, focus on atmospheric and magnetospheric science will be enabled through unique observations. In addition, together with Parker Solar Probe and Solar Orbiter, operational at the same time and also performing gravity assist maneuvers at Venus, ${ }^{3}$ and the ongoing Akatsuki - JAXA's Venus Climate orbiter mission (Nakamura et al. 2011) there is a unique opportunity for coordinated observations. ${ }^{4}$

Six Mercury flybys will take place from late 2021 to early 2025 (see Fig. 16), with various geometries that offer a variety of science investigations to be performed by instruments onboard the MPO and Mio spacecraft. Distances of the first four flybys will be on the order of $200 \mathrm{~km}$ from the surface, which is in contrast with the fifth flyby closest approach at $40.000 \mathrm{~km}$, and the last flyby at $400 \mathrm{~km}$. The flyby geometries will probe various parts of the exosphere and magnetosphere of Mercury, a critical aspect to improve the understanding of the dynamics of Mercury's environment.

In-situ measurement of cosmic dust including cometary dust trail particles during the cruise phase are possible (as e.g., achieved by the Helios spacecraft, Grün et al. 1975). A modeling of the dust trails of several cometary orbits using the Interplanetary Meteoroid Environment for Exploration (IMEX, see Soja et al. 2014) model will provide opportunity windows for BepiColombo to detect dust, e.g., by the Mercury dust monitor instrument or through analyzing the spacecraft attitude. For more details see Mangano et al. (2021), this journal.

\subsection{Science Payload}

BepiColombo has a comprehensive state-of-the-art suite of payloads covering the entire electromagnetic spectrum plus mass spectrometry and plasma physics. The total number of scientific sensors $(\approx 30)$ of the 12 instruments and 4 instrument suites onboard the two spacecraft, the high spectral and spatial resolutions of these sensors, and the two spacecraft flying in dedicated and coordinated orbits around Mercury will allow scientists to perform measurements to follow-up on the earlier findings of NASA's MESSENGER (Solomon

\footnotetext{
${ }^{3}$ Parker Solar Probe Venus gravity assist flybys: \#3 on 20 July 2020, \#4 on 20 February 2021, and \#5 on 16 October 2021; Solar Orbiter Venus gravity assist flybys: \#1 on 27 December 2020 and \#2 on 8 August 2021.

${ }^{4}$ The unique opportunity for coordinated observations of these spacecraft also exist outside the flybys and is followed up by the science teams of these missions.
} 

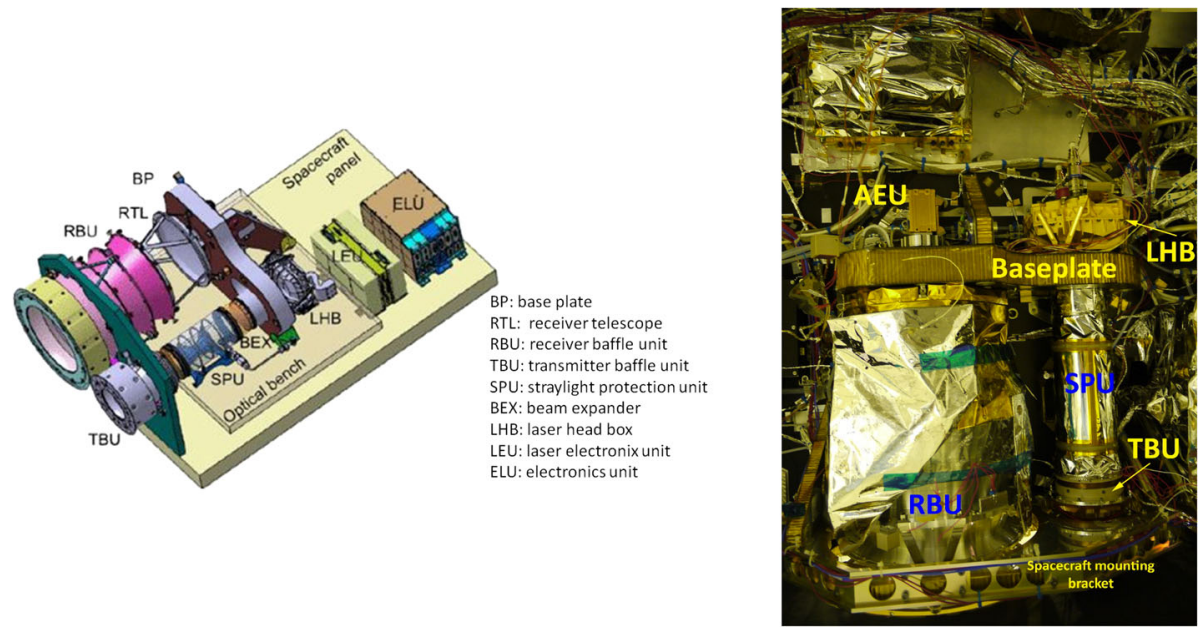

Fig. 17 BELA: instrument mass $\tilde{1} 5 \mathrm{~kg}$; power consumption $\tilde{4} 3.25 \mathrm{~W}$ (average at $10 \mathrm{~Hz}$ ) image taken from Thomas et al. (2021)

and Anderson 2018; McNutt et al. 2018) and Mariner 10 (Shirley 2003) missions with higher granularity and breadth. The MPO will focus on a global characterization of Mercury through the investigation of its interior, surface, exosphere, and magnetosphere. In addition, it will be testing Einstein's theory of general relativity. The scientific payload onboard the MPO (see Table 2) will provide detailed information necessary to better understand the process of planetary formation and evolution in the hottest part of the proto-planetary nebula as well as the similarities and differences between the magnetospheres of Mercury and the Earth. The Mio spacecraft instruments (see Table 3) are more focused on studying the plasma, particle, and magnetic environment around the planet. BepiColombo will therefore provide a rare opportunity to collect multi-point measurements in a planetary environment. This will be particularly important at Mercury because of the short temporal and spatial variability scales of processes in Mercury environment. The currently planned orbits will make possible close encounters of the two spacecraft throughout the mission. Such intervals are very important for the inter-calibration of similar instruments on the two spacecraft.

\subsection{MPO payload description}

The MPO hosts several scientific instruments that are described in alphabetical order.

The BepiColombo Laser Altimeter (BELA, Fig. 17) instrument's scientific goals are characterizing and measuring the figure, topography, and surface morphology of Mercury. BELA will provide absolute topographic elevation and position with respect to a Mercury-centred coordinate system. This information will be used to create digital terrain models that allow the inversion of the gravity field from Mercury data, acquired by the MORE instrument, for internal structure and to quantitatively explore the geology and the tectonics of the planet's surface. BELA will aim at measuring Mercury's tidal deformation and will provide constrains on Mercury's rotation state. The returned laser signal can be used to measure the local surface roughness and the albedo at the laser wavelenght of $1064 \mathrm{~nm}$. In synergy with other instrument results, it will also be possible to explore the interior structure and the geologic evolution of the planet. For more details see Thomas et al. (2021). 
Fig. 18 ISA: instrument mass $\tilde{7} .5 \mathrm{~kg}$; power consumption 22.15 W (average)

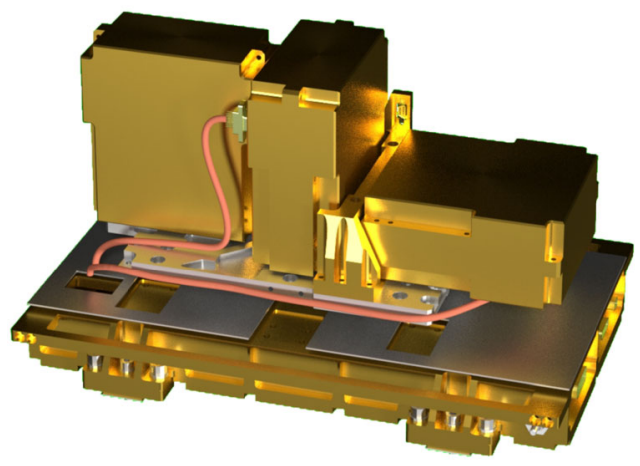

Fig. 19 MPO-MAG: Sensor fight models (left and right in front) with electronics box (black, center) and the thermal housing (in the back). Instrument mass $\tilde{2} .5 \mathrm{~kg}$; power consumption 5.0 W (average); Image taken from Heyner et al. (2021)

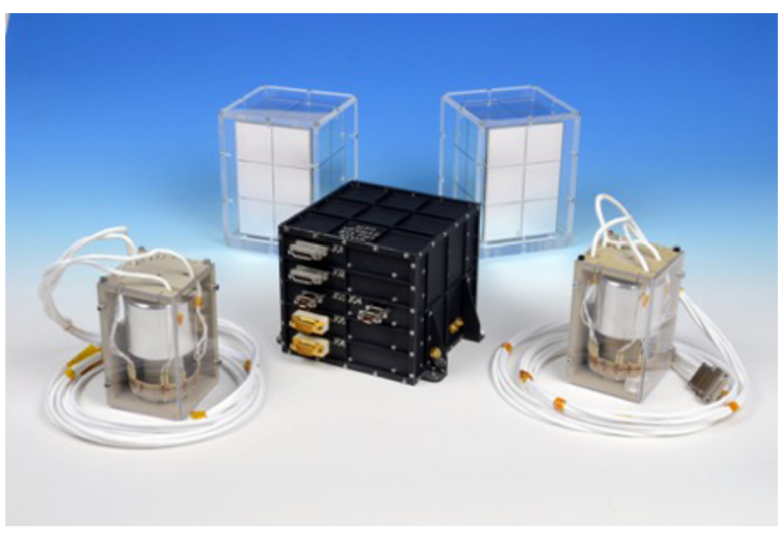

The Italian Spring Accelerometer (ISA, Fig. 18) is an high-sensitivity, three-axis accelerometer. It is one of the instruments contributing to the BepiColombo Radio Science Experiments dedicated to the characterization of planet Mercury geodesy and geophysics, as well as to testing Einstein theory of general relativity. ISA will provide measurements of the non-gravitational perturbations the MPO will be subject to in its orbit around Mercury. This information will be integrated in the determination of the MPO orbit. The availability of such measurements will allow to reach an higher accuracy orbit determination with respect to the traditional methods (i.e. without an on-board high-sensitivity accelerometer) and to distinguish between gravitational and non-gravitational forces acting on the spacecraft. For more details see Santoli et al. (2020).

The primary objective of the MPO Magnetometer (MPO-MAG, Fig. 19) is to collect magnetic field measurements in order to describe Mercury's planetary magnetic field and its source in unprecedented detail. This will help us understand the origin, evolution and current state of the planetary interior. The requirement is to determine all the terms associated with the internal field up to the octopole with high accuracy, using accurate magnetic field measurements on the low orbit of MPO. This will be supported by similar measurements made on Mio, in order to distinguish the effects of the magnetospheric currents on the MPO measurements and to use the Mio measurements directly to augment the database for the determination of the internal terms. The secondary objectives of MPO-MAG are related to the interaction of the solar wind with Mercury's magnetic field and the planet itself. This interaction leads to the formation of the global magnetospheric current systems that are highly 
Fig. 20 MERTIS: instrument mass $\tilde{3} .3 \mathrm{~kg}$; power consumption 10.0 - $12.4 \mathrm{~W}$ (average); Image taken from Hiesinger et al. (2020)

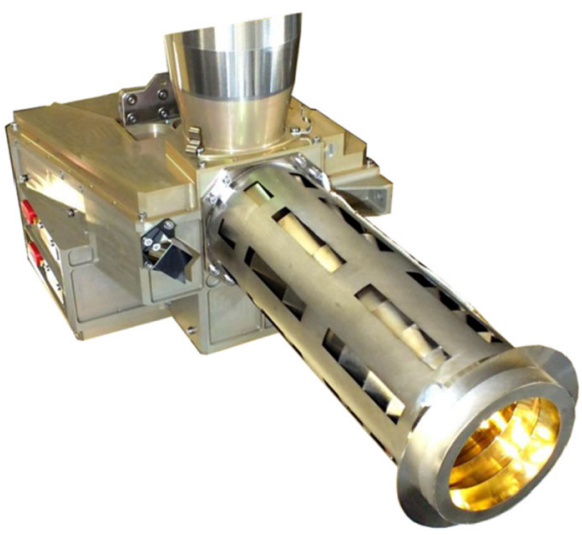

dynamic. In particular, measurements close to the planet will allow the determination of the conditions for access of the solar wind to the planetary surface and assessing the role and importance of different current systems, including subsurface induction currents and the conductivity of the regolith (Anderson et al. 2014). These objectives will again be assisted by the planned close association with the magnetic field investigation on the Mio. The MPO-MAG experiment consists of a dual fluxgate magnetometer system that will measure 3-D magnetic fields from DC up to $128 \mathrm{~Hz}$ within $\pm 2048 \mathrm{nT}$ with a digital resolution better than $60 \mathrm{pT}$. In order to determine magnetic contamination (AC and DC) from the spacecraft, MPO-MAG consists of two sensors, an inboard and an outboard sensor, mounted on an about $3 \mathrm{~m}$ long boom and separated by $80 \mathrm{~cm}$. For further details of the experiment, see Heyner et al. (2021).

The goal of the MErcury Radiometer and Thermal Infrared Spectrometer (MERTIS, Fig. 20) is to provide detailed information about the mineralogical composition of Mercury's surface by globally mapping spectral emittance with high spectral resolution. Knowledge of mineralogical composition is crucial for choosing the best of several competing theories, and thus for selecting a valid model for the origin and evolution of the planet. For example, if a giant impacts stripped off the primordial crust and upper mantle soon after Mercury has been formed, models predict that the present-day surface is composed of rocks highly depleted in those elements that were initially concentrated in the primordial crust (Benz et al. 1988). MERTIS has four main scientific objectives: 1) the study of Mercury's surface composition, 2) identification of rock-forming minerals, 3) mapping of the surface mineralogy, and 4) the study of surface temperatures and thermal inertia. MERTIS covers the wavelength range from 7 to $14 \mu \mathrm{m}$ with a spectral resolution of up to $90 \mathrm{~nm}$. This allows detection and identification of the characteristic features of the surface minerals in this spectral region, such as the Christiansen feature, Reststrahlen bands, and Transparency features. In addition, MERTIS will be able to measure thermophysical properties of the surface like thermal inertia and surface texture. MERTIS is an IR-imaging spectrometer, which will make use of the micro-bolometer technology where no cooling is required. MERTIS will globally map the planet with a spatial resolution of $500 \mathrm{~m}$. For more details see Hiesinger et al. (2020).

The scientific goals of the Mercury Gamma-Ray and Neutron Spectrometer (MGNS, Fig. 21) are to determine the elemental compositions of distinguishable regions over the entire surface of Mercury. The elemental composition will be determined by the measurements of nuclear lines of major soil-composing elements $\mathrm{Si}, \mathrm{O}, \mathrm{C}, \mathrm{Al}, \mathrm{Na}, \mathrm{Fe}, \mathrm{Ca}, \mathrm{S}, \mathrm{Cl}$ and natural radioactive elements $K, T h, U$ in the spectra of gamma-rays from the planetary 

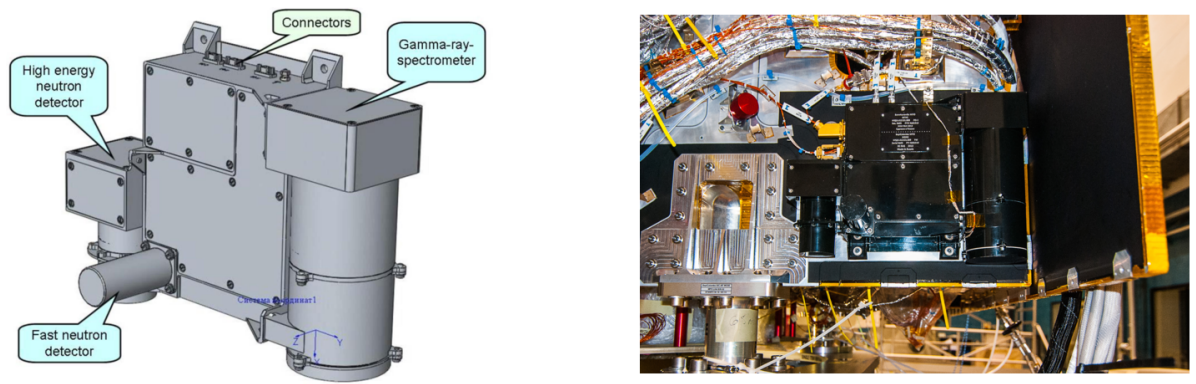

Fig. 21 MGNS installed on the MPO spacecraft: instrument mass $\tilde{5} .5 \mathrm{~kg}$; power consumption $\tilde{6} .4 \mathrm{~W}$ (average); Image taken from Mitrofanov et al. (2021)

surface with an accuracy about $10-30 \%$ and with a surface resolution of about $400 \mathrm{~km}$ at the pericenter of the MPO orbit. A secondary objective is to determine the regional distribution of hydrogen (most likely related to water ice) depositions on the both Northern and Southern polar areas of Mercury, which are permanently shadowed from the Sun and to provide a map of the column density of these depositions with the accuracy of $0.1 \mathrm{~g} / \mathrm{cm}^{2}$. Since Mercury lacks an atmosphere and has only a weak magnetic field heavy particles (like $\mathrm{Fe}$ with a extremely short stopping length) of galactic cosmic rays (GCRs) propagate to and interact with its shallow surface. Due to the bombardment by GCRs, a large number of secondary high energy neutrons are produced within a subsurface layer of 1 to $2 \mathrm{~m}$ thickness. These neutrons interact with nuclei of atoms of the subsurface material, producing emission of gamma rays and leakage flux of moderated neutrons. Each soil-composing element has a unique set of nuclear lines of gamma-rays, so that gamma-ray spectroscopy can identify the composition of the Mercury subsurface. Lines of natural radioactive isotopes of $K, T h$ and $U$ are produced independent of the bombardment by GCRs cosmic rays. So, the surface elemental composition of particular regions of the planet can be studied from observations of these nuclear gamma-ray lines. The energy spectrum of leakage neutrons also depends on the presence of hydrogen in the subsurface material due to its high efficiency of moderation. Suppression of surface emission of epithermal neutrons is a rather sensitive signature of enhancement of hydrogen in the subsurface material. Measurements of such suppressions at polar regions allow one to map the water ice deposits at both poles of Mercury. The MGNS instrument is based on two separate segments of individual detectors: The Mercury gamma ray spectrometer, uses the innovative $\mathrm{CeBr}_{3}$ scintillator with rather high spectral resolution of $\tilde{3} \%$ (Kozyrev et al. 2015, 2016a,b), and the Mercury neutron spectrometer, which consists of a scintillation detector of high energy neutrons and the set of three sensors of thermal and epithermal neutrons. For more details see Mitrofanov et al. (2021), Mitrofanov et al. (2010) and Kozyrev et al. (2018).

The Mercury Imaging X-ray Spectrometer (MIXS, Fig. 22) is designed to perform X-ray fluorescence (XRF) analysis of the surface of Mercury. XRF is a well-known technique ((van Grieken and Markowicz 1993) used for remote sensing of the atomic composition of airless, inner Solar System bodies. The primary scientific goal of MIXS is to produce global elemental abundance maps of key rock-forming elements to an accuracy of 5-50\% depending on concentration. If solar conditions permit, high spatial resolution mapping of these elemental abundances will be accomplished. A third scientific objective is to confirm whether the auroral zone, where energetic particles interact with the surface, is an intense source of continuum and line X-rays. Achieving these objectives will allow to address key science 
Fig. 22 MIXS: instrument mass $11.4 \mathrm{~kg}$; power consumption 19.3 W (average)
Fig. 23 MORE-Kat: fully digital transponder, mass $\tilde{3} .5 \mathrm{~kg}$; power consumption $\tilde{3} 2.6 \mathrm{~W}$ (average)
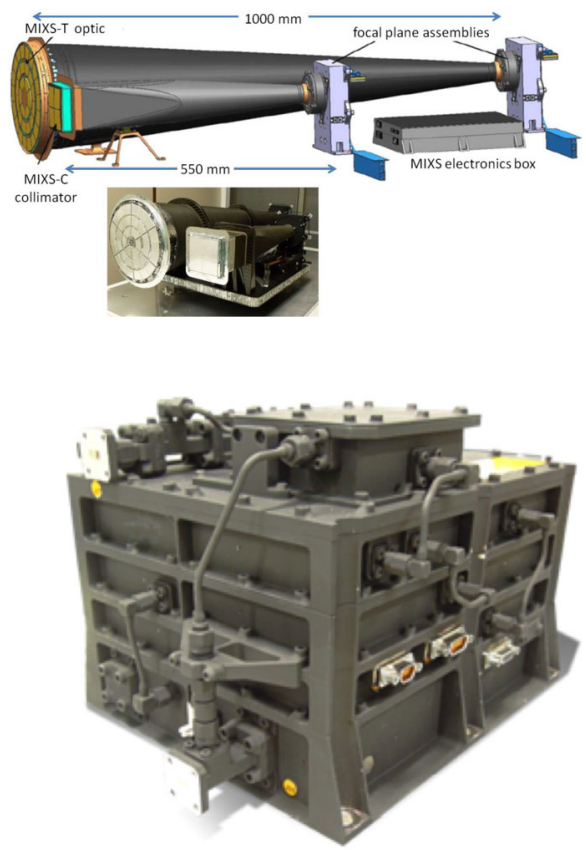

issues of the origin of Mercury like the evolution, the nature of surface modifications (e.g., cratering, volcanism), and the structure and variation of the magnetosphere of Mercury. All orbital XRF experiments rely on solar coronal X-rays to provide the primary illumination. The Sun excites $\mathrm{K}$ and $\mathrm{L}$ shell fluorescence line emission in the top few microns of the surface, which is characteristic of the surface abundance. XRF in the $0.5-7.5 \mathrm{keV}$ energy range allows the absolute quantification of the elemental abundances of light, rock forming elements - from $\mathrm{Mg}$ to $\mathrm{Fe}$. A prerequisite for achieving this absolute quantification is the full characterization of the highly variable incident solar flux. MIXS will obtain this reference information from its partner instrument, SIXS. In order to achieve its science objectives, MIXS consists of two channels - the MIXS-C, a collimator providing efficient flux collection over a broad range of energies with a wide field of view for planetary mapping and the MIXS-T, an imaging telescope with a narrow field for high-resolution measurements of the surface. Operation of the MIXS instrument will take place continuously on the day- and night-side. Night side observations will be possible as there is a large flux contribution expected from auroral arcs at Mercury (Lindsay et al. 2016). For more details see Bunce et al. (2020).

The Mercury Orbiter Radio-science Experiment (MORE) addresses scientific goals in geodesy, geophysics, and fundamental physics. It will help to determine the gravity field of Mercury as well as the size and physical state of its core. It will provide crucial experimental constraints to models of the planet's internal structure and test theories of gravity with unprecedented accuracy. MORE will also measure the gravitational oblateness of the Sun (Damiani et al. 2011) and test and characterize the most advanced interplanetary tracking system ever built. Finally, it will assess the performance of the novel tracking system in accurate orbit determination and space navigation. These scientific goals will be achieved by means of several data types, generated by MORE itself at the ground station, other onboard instruments (BELA, ISA, and SIMBIO-SYS), and the onboard attitude determination 

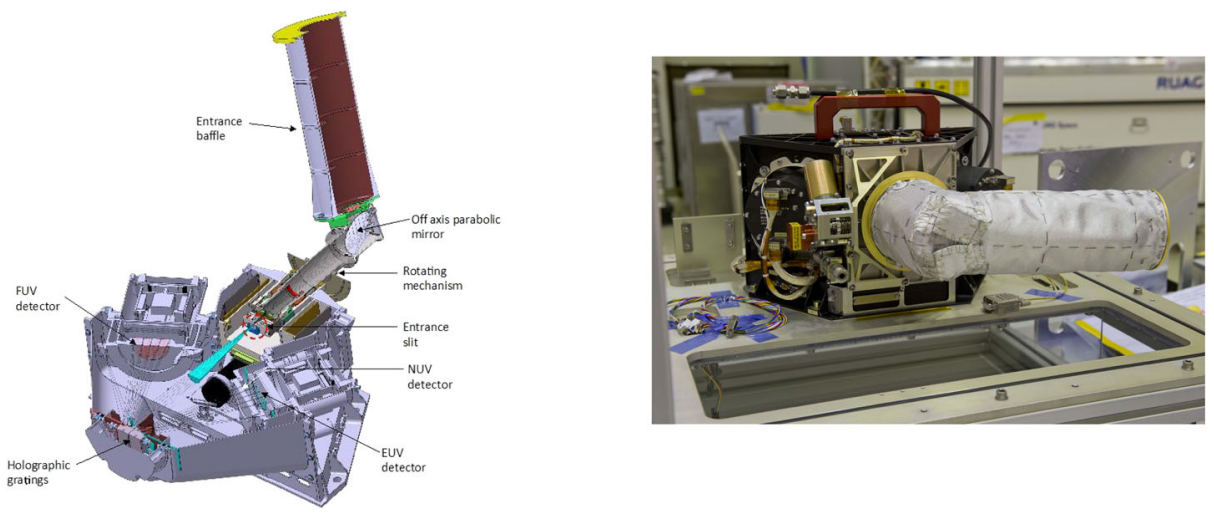

Fig. 24 PHEBUS: instrument mass $\tilde{7} .6 \mathrm{~kg}$; power consumption $\tilde{4} .0 \mathrm{~W}$ (average)

and control system. MORE will also contribute to the determination of Mercury's obliquity (i.e., the obliquity of the equator with respect to the orbital plane) and the amplitude of its 88-day physical librations in longitude. These two quantities, together with the coefficients of the second-degree harmonics of the gravity field, will confirm whether or not Mercury has a molten core and will provide the radius of this molten core. The key instrument is an advanced Ka-band transponder (KaT, see Fig. 23), which ensures the on-board reception of a Ka-band radio signal and its coherent retransmission to Earth. This instrument does not produce any telemetry, as the measurements are actually carried out by the ground station.

The basic observables produced by MORE are range, with an accuracy of $15 \mathrm{~cm}$, and range rate, with an accuracy of $1.5 \mu \mathrm{m} / \mathrm{s}$ at $1000 \mathrm{~s}$ integration time, exploiting the microwave radio links to and from the spacecraft. The range rate is measured from the Doppler shift of an electromagnetic wave transmitted from ground, received by the spacecraft and coherently re-transmitted back to ground. The spacecraft range is obtained from the measurement of the time delay between transmission and reception of a known modulation of the carrier. MORE data will be combined with the measurements of the non-gravitational acceleration by the BepiColombo Accelerometer ISA to generate an orbital solution and the estimate of the physical parameters of interest. SIMBIO-SYS high-resolution images will be used for the optical tracking of surface landmarks in order to determine the rotational state of the planet. For more details see Iess et al. (2021).

PHEBUS (Probing of Hermean Exosphere by Ultraviolet Spectroscopy, Fig. 24) is an Ultra Violet (UV) spectrometer working in the wavelength range from $55 \mathrm{~nm}$ (Extreme Ultra Violet, EUV) to $330 \mathrm{~nm}$ (Far Ultra Violet, FUV) and devoted to the characterisation of Mercury's exosphere composition and dynamics. Two gratings and two detectors are used in a special, innovative, compact design. A one degree-of freedom scanning system allows probing, at the highest possible signal-to-noise ratio of selected regions and altitude ranges of interest. The spectral range of PHEBUS contains the major resonance lines of most expected or detected species. The general scientific objectives are oriented toward better understanding of the coupled surface-exosphere-magnetosphere system, like determination of the composition and vertical structure of the exosphere including previously undetected species, day-to-night circulation, study of surface release processes, identification and characterisation of the sources, space and time monitoring of exosphere/magnetosphere exchange and transport processes, and study of global scale source/sink balance and geo-chemical cycles.

Mercury's exosphere is expected to vary rapidly in response to solar wind variations, and therefore it is important to provide partial maps of the exosphere on time scales of less than 

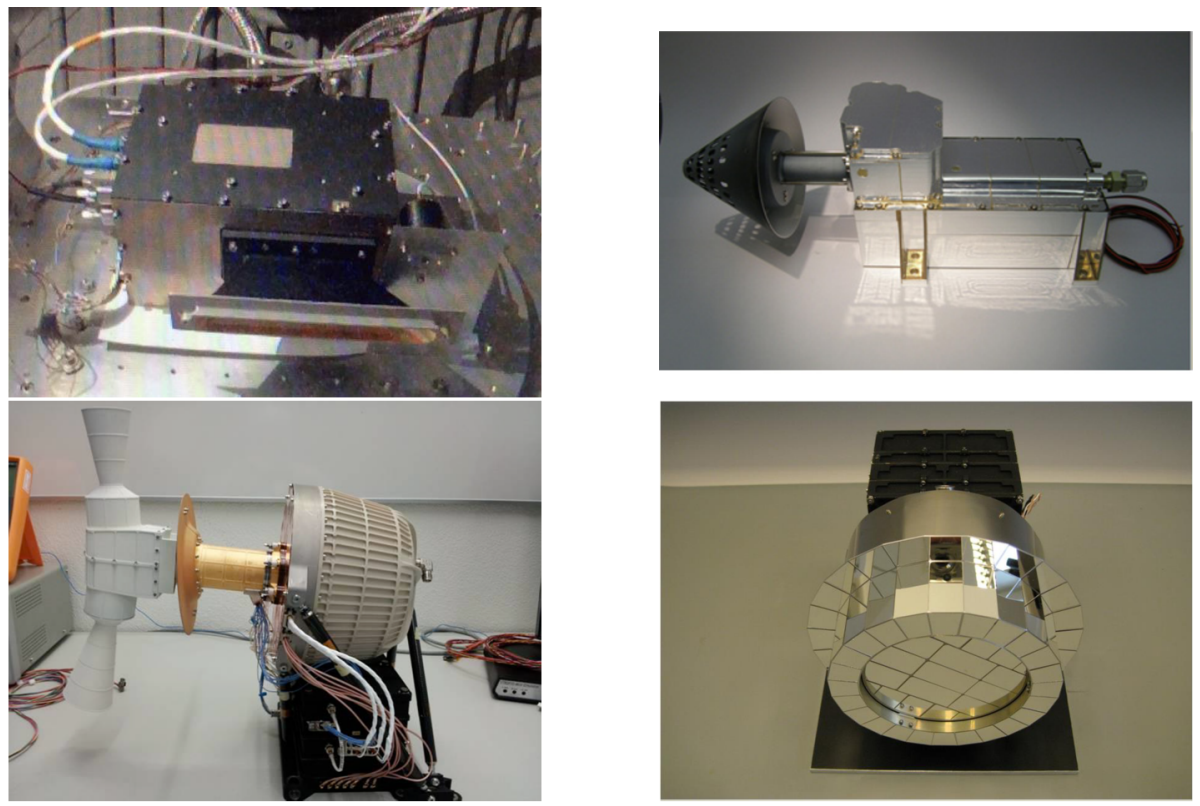

Fig. 25 SERENA instrument suite: ELENA, MIPA, STROFIO, and PICAM (left to right); total mass $\tilde{9} .7 \mathrm{~kg}$; power consumption $\tilde{3} 4.5 \mathrm{~W}$ (average)

a few hours. The polar orbit of the spacecraft will allow the exosphere to be monitored at all latitudes but only within a narrow longitudinal region along the orbit, with the restriction that only regions of the exosphere illuminated by the Sun may be observed. The instrument is composed of two Ultra-Violet spectro-photometers and one scanning mirror (one axis rotation). The EUV spectrograph covers a spectral range of $55-155 \mathrm{~nm}$, which allows it to catch second order lines in the $25-50 \mathrm{~nm}$ range. The second spectrometer, FUV, can measure a spectral range of $145-315 \mathrm{~nm}$ with an extension at 404 and $422 \mathrm{~nm}$ in the Near Ultra Violet (NUV). Together, they yield a spectral resolution of $1 \mathrm{~nm}$. PHEBUS receives the UV emission through a baffle mounted through the radiator at an angle of $10^{\circ}$ and can rotate by $360^{\circ}$ in one plane. This allows one-degree of freedom in pointing, enabling scanning of selected regions and altitude ranges of interest. For more details see Quémerais et al. (2020).

The SERENA (Search for Exospheric Refilling and Emitted Natural Abundances, Fig. 25) experiment will provide information on the whole surface-exosphere-magnetosphere coupled system and the processes involved plus the interactions between energetic particles, the solar wind, micrometeorites, and the interplanetary medium. The experiment consists of four sensors that can be operated individually: 1) ELENA (Emitted Low-Energy Neutral Atoms) is a neutral particle camera that investigates neutral gases escaping from the surface of Mercury, their dynamics, and the processes responsible for such a population. ELENA has a 1-D field of view for mapping neutral particle released from the surface; the second dimension of the data will be provided by the movement of the spacecraft. ELENA's field of view (FOV) is $4.5^{\circ} \times 76^{\circ}$, directed towards the surface of Mercury (nadir) with an angular resolution of $4.5^{\circ} \times 4.5^{\circ}$. ELENA measures energetic neutrals between $20 \mathrm{eV}$ and $5 \mathrm{keV}$. 2) STROFIO (STart from a ROtating Field mass spectrOmeter) is a neutral particle spectrometer, which investigates the exosphere gas composition. It is a novel type of mass spectrometer. The neutral particles enter into the system and become ionized. The ions are released into the 

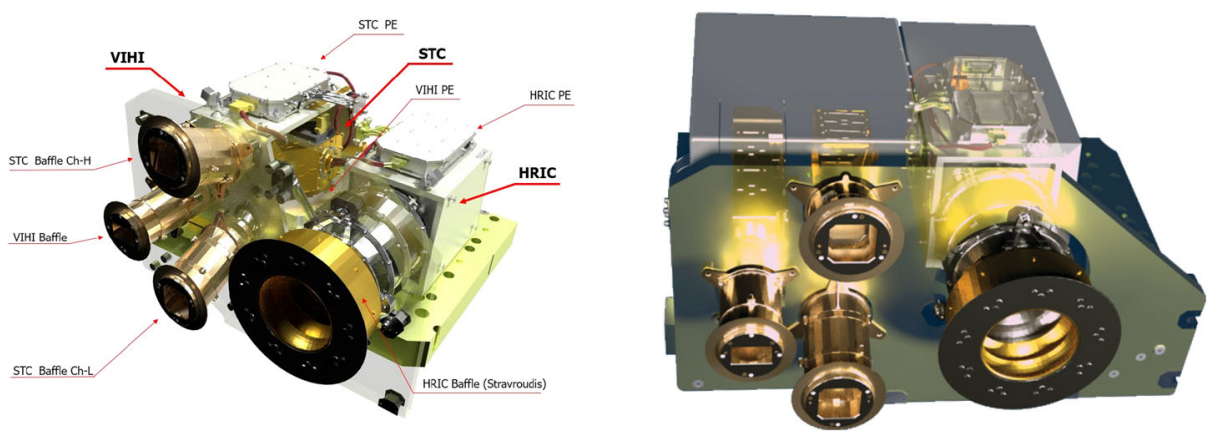

Fig. 26 SIMBIO-SYS: instrument suite mass $\tilde{1} 4.8 \mathrm{~kg}$; power consumption $\tilde{2} 3.4 \mathrm{~W}$ (average). Acronyms are explained in the text

focusing optic and move on a constant trajectory to the 2D detector system where the time of flight is measured, from which the mass/charge can be calculated. 3) MIPA (Miniature Ion Precipitation Analyser) is an ion monitor investigating the chain of processes by which plasma precipitates towards the surface. It consists of a sensor and a single electronics board including a high voltage supply and front-end-electronics. The ion flux arrival angle is analyzed by an electrostatic deflector and the energy by a following electrostatic analyzer. The geometric factor of MIPA is optimised for monitoring very high fluxes of the precipitating solar wind and magnetospheric ions that may eventually induce the ion-sputtering process. It has a $2 \pi \mathrm{FOV}$, a pure ion mass resolution and can detect one direction at $10 \mathrm{eV}-15 \mathrm{keV}$ energies. 4) PICAM (Planetary Ion Camera) is an ion mass spectrometer operating as an all-sky camera for charged particles to study the chain of processes by which neutrals are ejected from the soil, ionised, and transported through the environment of Mercury. The ion optics is based on the principle of a modified pin-hole camera. The sensor is axisymmetric along the $\mathrm{Z}$ axis and its field of view is a hemisphere centred along this axis. Its energy resolution is $1 \mathrm{eV}-3 \mathrm{keV}$. In summary, STROFIO measures in-situ the lower-energies neutral particle composition and density in the exosphere; ELENA covers the higher energy spectrum of neutral particles released from the surface; MIPA will monitor the solar wind, whereas PICAM is optimized to measure low-energy planetary ions. Each unit is able to operate individually to achieve specific scientific objectives of its own; however, operating simultaneously greatly improves the chances of success of scientific objectives and allows for additional objectives. For more details see Orsini et al. (2021).

The SERENA and PHEBUS observations are both complimentary and highly supportive of each other, as PHEBUS will produce global maps of the exosphere and SERENA will measure in-situ both neutrals and ions. The key scientific objectives of SERENA include the identification and localization of source and sink processes of neutral and charged particles as well as estimates of their relative efficiencies. The latter depends on surface composition and external drivers such as solar irradiance or plasma precipitation, and so both spatial and temporal variability are expected. It further includes the composition and spacial distributions of neutrals and ions in the exosphere for all species, including their energy spectra. The dynamics of the neutral and ionized exosphere: day-to-night circulation, active-to-inactive regions, etc., will be investigated as well as atmosphere/magnetosphere exchange and transport processes.

SIMBIO-SYS (Spectrometer and Imagers for MPO BepiColombo Integrated Observatory System, Fig. 26) is an integrated suite for the imaging and spectroscopic investigation of the surface of Mercury. The science goals of SIMBIO-SYS are to examine the surface geology 

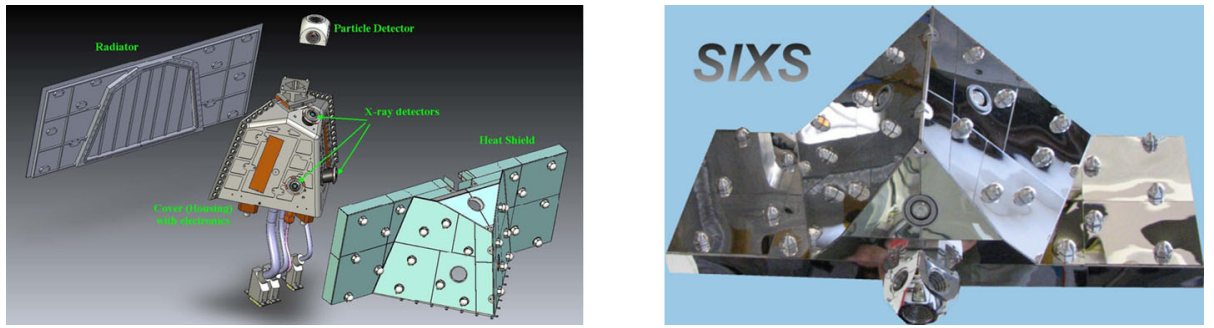

Fig. 27 SIXS: instrument mass $\tilde{2} .4 \mathrm{~kg}$; power consumption $\tilde{5} .3 \mathrm{~W}$ (at $+20{ }^{\circ} \mathrm{C}$ ambient temperature). The power consumption of the SIXS share in the MIXS Electronics Box is $\tilde{7} .7 \mathrm{~W}$

(stratigraphy, geomorphology), volcanism (lava plain emplacement, volcanoes identification), global tectonics (structural geology, mechanical properties of the lithosphere), surface age (crater population and morphology, degradation processes), surface composition (maturity and crustal differentiation, weathering, rock-forming-minerals abundance determination), and geophysics (libration measurements, internal planet dynamics) of Mercury. It incorporates capabilities to perform medium spatial resolution global mapping in stereo and color using two pan-chromatic and 3 broad-band filters, respectively. SIMBIO-SYS will also acquire high spatial resolution imaging in one panchromatic and three broad-band filters and imaging spectroscopy in the spectral range of 400-2000 nm. SIMBIO-SYS consists of 3 independent units: 1) The Stereo Channel (STC) will provide global color coverage of the surface in full stereo at $58 \mathrm{~m} /$ pixel resolution at $480 \mathrm{~km}$ from the planet, generating the Digital Terrain Model of the entire planet. It will be a useful tool to define with high detail the topography, which is a critical measurement for tectonic features characterization, geological units lateral boundaries definition, and for measuring important geophysical parameters. STC will provide color images of selected regions using 4 broad-band filters (420, 550,700 , and $880 \mathrm{~nm}$ ). The stereo acquisition of STC is based on the push-frame design. The FoV cross track of STC is $5.3^{\circ}$ and the stereo angle is $40^{\circ}$. There are no moving parts. 2) The High spatial Resolution Imaging Channel (HRIC) will provide images with a pixel scale of $6 \mathrm{~m} /$ pixel, at $480 \mathrm{~km}$ from the planet, of selected regions for about $20 \%$ of the surface in the first year of the mission. The regions will be observed with the panchromatic filter and/or the 3 broad-band filters $(550,750,880 \mathrm{~nm})$. HRIC is a very compact design based on the Ritchey-Chretien optical concept. The FoV cross track is $\left.1.47^{\circ} .3\right)$ The Visible Infrared Hyperspectral Imager Channel (VIHI) is a hyperspectral imaging push-broom spectrometer, with a FoV cross track of $3.7^{\circ}$ in the spectral range of 400-2000 $\mathrm{nm}$. It will provide the global mineralogical composition of the surface with a spectral sampling of $6.25 \mathrm{~nm}$ and a pixel scale of $500 \mathrm{~m} /$ pixel at $480 \mathrm{~km}$ from the surface. Selected regions will be observed with a pixel scale down to $120 \mathrm{~m} /$ pixel at $480 \mathrm{~km}$ from the surface. VIHI is based on a strong heritage of VIRTIS that was on board the Rosetta mission (Coradini et al. 1998) and other planetary missions. The three channels share a common electronics box. However each channel has its own proximity electronic (PE) box. For more details see Cremonese et al. (2020).

The SIXS (The Solar Intensity X-ray and particle Spectrometer, Fig. 27) experiment will perform measurements of X-rays and particles of solar origin at the position of BepiColombo. The instrument is capable of broadband measurements of X-ray, proton, and electron spectra with high time resolution and a very wide FoV. The surface of Mercury is bombarded with intensive solar X-rays and particle radiation, which process makes the Hermean surface glow in X-rays. Changes of the input radiation are due to solar activity and eruptive 
Fig. 28 MDM: instrument mass

$\tilde{0} .9 \mathrm{~kg}$; power consumption

4.6 W (nominal)

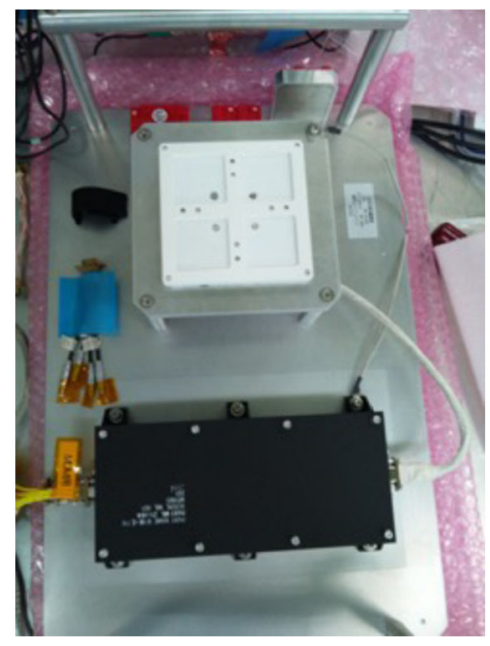

phenomena on the Sun. The radiation varies unpredictably with time scales from seconds to years and several orders of magnitude in intensity. The X-ray glow of Mercury provides a "Hermean signature" in the form of fluorescence. The primary task of SIXS is to make observations enabling accurate physical estimates of solar X-ray, proton, and electron irradiation at the surface of Mercury. The surface is simultaneously observed by the Mercury Imaging X-ray Spectrometer (MIXS), a closely linked instrument. Data provided by SIXS are mandatory for a valid fluorescence analysis of MIXS spectra. The solar X-ray monitor is also the single source of these necessary data in orbital phases of Mercury while the planet is behind the Sun. The scientific objectives of SIXS are studies of temporal and spectral variability of solar X-ray corona, temporal behavior and X-ray spectral classification of solar flares, and temporal and spectral variability of proton and electron radiation near Mercury.

SIXS is capable of performing measurements of X-ray spectra (SIXS-X) with time resolution down to $1 \mathrm{~s}$ in the energy range $1-20 \mathrm{keV}$ and simultaneous proton and electron spectra (SIXS-P) in the energy range $0.33-30 \mathrm{MeV}$ for protons and $50 \mathrm{keV}-3 \mathrm{MeV}$ for electrons. Both X-ray and particle channels are capable of measuring count-rates up to $20000 \mathrm{cps}$ with very small pile-up, and the channels both have a low background. Both channels have a total FoV of at least $180^{\circ}$ degrees in diameter. The instrument has on-board radioactive sources $\left(\mathrm{Fe}^{55}\right)$ for spectral calibration of the X-ray detectors. For more details see Huovelin et al. (2020).

\subsection{Mio Payload description}

MDM (Mercury Dust Monitor, Fig. 28) is an instrument to explore the dust environment at Mercury's region of the solar system $(0.31-0.47 \mathrm{AU})$. It will measure impact momentum, crude direction and the flux of the dust around Mercury. At Mercury's position, the main components of dust particles are Keplerian dust particles and $\beta$-meteoroids. The Keplerian dust particles are so-called interplanetary dust particles (IPDs) that originate from asteroids or comets and gradually decrease their solar-centric distance by the PoyntingRobertson effect. Those dust particles bombard Mercury's surface and impact ejecta particles are launched to higher altitude, and the particles with less than Mercury's escape velocity fall down to the surface. The bombardment of micro-meteoroids continuously occurs on 

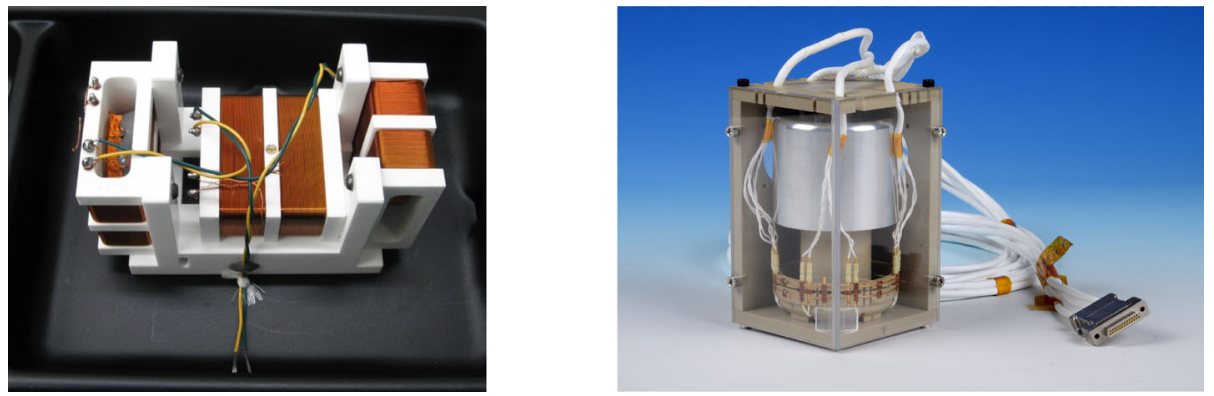

Fig. 29 MGF inboard sensor and MGF outboard sensor in protection housing: total instrument mass $\tilde{0} .77 \mathrm{~kg}$; power consumption $\tilde{4} .4 \mathrm{~W}$ (images taken from Baumjohann et al. 2020)

air less bodies, and the impact ejecta particles form a so-called "dust cloud" around the body (Krüger et al. 1999, 2000), so the same may apply to Mercury. The bombardment of micrometeoroids may also contribute significantly as one of the source processes of the planet's tenuous atmosphere. The MDM system is composed of a $64 \mathrm{~cm}^{2}$ piezoelectric PZT sensor unit (MDM-S) attached to the outside of the side panel and the electronics unit (MDM-E) installed inside the spacecraft. The viewing direction almost covers a half-sphere. PZT is a very simple device that can stand high temperatures (about $+170{ }^{\circ} \mathrm{C}$ ) and does not need to apply any high voltage to the sensor as bias voltage for operation. For more details see Kobayashi et al. (2020).

MGF (Magnetic Field Investigations, Fig. 29) is a set of magnetometers aboard Mio to perform magnetic field measurements. Understanding the magnetosphere of Mercury is important both for its own sake as well as in comparison with those known from other planetary bodies (Earth, Jupiter, and Saturn). The solar-wind speed at Mercury's orbit is almost the same as at Earth, but the magnitude of the interplanetary magnetic field (IMF) is increased about five times and the solar-wind density by close to a factor of ten. Typical processes in the Hermean magnetosphere occur much faster, by about a factor of 30, than at Earth, and so measurements need to be taken at a much faster rate than typically done in the Earth's magnetosphere (Anderson et al. 2010). Hence, MGF will use a $128 \mathrm{~Hz}$ sampling rate at maximum. Magnetic field observations with high temporal resolution will help to clarify the role of magnetic fluctuations in solar wind heating and acceleration. MGF is also well-prepared to assist the sister magnetometer MPO-MAG aboard the Mercury planetary orbiter in measuring Mercury's intrinsic magnetic field, in particular by helping to distinguish between temporal fluctuations and spatial variations. MGF consists of two sets of fluxgate magnetometers, MGF-O for the outer sensor and MGF-I for the inner sensor where MGF-O is a so-called digital-type and MGF-I is a traditional analogue-type magnetometer. Both have their own sensor and electronic board. The outer sensor is mounted on the tip of a $4.4 \mathrm{~m}$ long boom, while the inner sensor is mounted at $1.6 \mathrm{~m}$ distance from the boom tip. The instrument is designed to measure magnetic fields with an accuracy of about $10 \mathrm{pT}$, a dynamic range of $\pm 2048 \mathrm{nT}$, and a time resolution of up to $128 \mathrm{~Hz}$. The selection of sampling rate of the data transmitted to the Earth is flexible and can be adapted to study each particular process in the observation regions. For more details see Baumjohann et al. (2020).

MPPE (Mercury Plasma Particle Experiment, Fig. 30) is a comprehensive instrument package for plasma, high-energy particle, and energetic neutral atom measurements. It consists of seven sensors. Six sensors perform in-situ observations and cover the charged particle species and the energy range of interest from the space plasma physics point of view. 


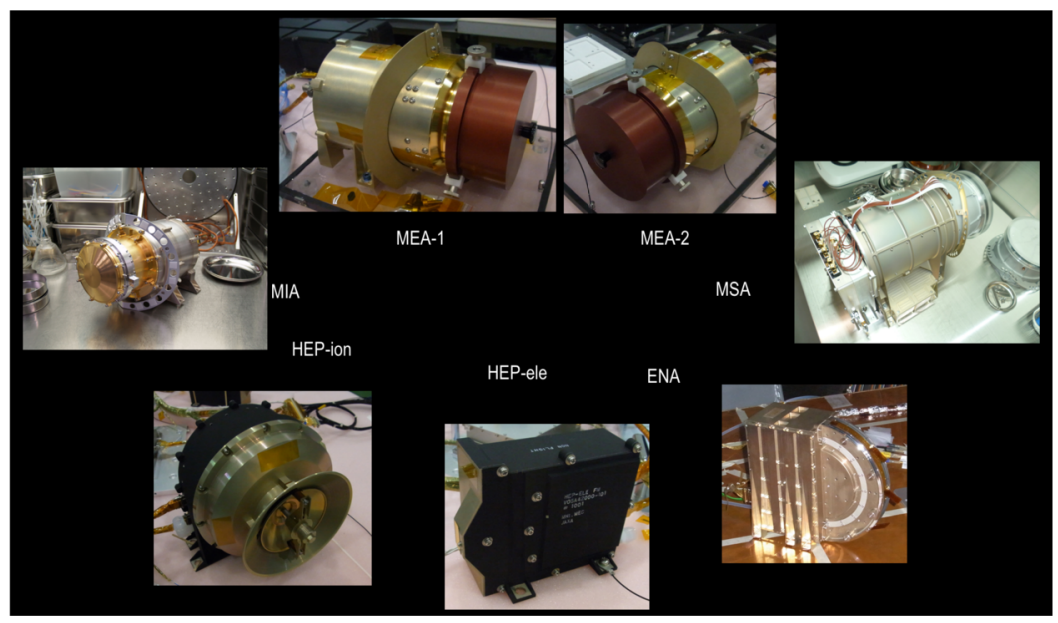

Fig. 30 MPPE instrument suite: total mass $\tilde{1} 2.8 \mathrm{~kg}$; power consumption 20.6 (average) W

Two Mercury Electron Analyzers (MEA1 and MEA2) mounted 90 apart are used for highresolution and a High Energy Particle instrument(HEP-ele) for electron coverage, and a Mercury Ion Analyzer (MIA), a Mercury mass Spectrum Analyzer (MSA), and a High Energy Particle instrument (HEP-ion) are used for ion coverage. The final sensor, Energetic Neutrals Analyzer (ENA), will detect energetic neutrals created via charge-exchange and will provide the scientists with remote information on how plasma and neutral gas interacts in the Hermean environment. Science goals of MPPE are to investigate the structure, dynamics, and physical processes in Mercury's magnetosphere, to study the formation and characteristics of the small-scale magnetosphere and the Solar wind contribution to the magnetospheric plasmas, and to investigate the interactions between surface, exosphere, and magnetosphere. The interaction between the solar wind and Mercury's magnetosphere is unique for many reasons. For example, due to the closeness of the Sun and the weak intrinsic magnetic field of Mercury, its magnetosphere is severely compressed; the estimated distance between the subsolar magnetopause and the surface is less than half the planetary radius. This could mean that sometimes the solar wind can directly interact with the planetary surface. Even if this does not happen, the plasma at the cusp can always interact with the surface, since there is no strong mirror reflection due to the smallness of the mirror ratio. How this kind of direct interaction with the surface affects the rest of the magnetospheric processes is an intriguing question that can never be tested in other planetary magnetospheres. MPPE will measure plasma, high-energy particles, and energetic neutral atoms around Mercury. MPPE will also make simultaneous measurements of high-energy electrons and ions, which is important in understanding the charged particle acceleration mechanism in a small-scale magnetosphere. For more details see Saito et al. (2021).

MSASI (Mercury Sodium Atmospheric Spectral Imager, Fig. 31) is a spectrometer devoted to the measurement of the narrow sodium D2 emission line $(589 \mathrm{~nm} \pm 0.028 \mathrm{~nm})$ against the bright surface of Mercury. MSASI will observe the north-south distribution and measure the temporal variations of the release rate of sodium atoms. Through these capabilities, it will be possible to investigate the source processes. Direct exposure of Mercury's rocky surface to the space environment gives the planet distinct characteristics in its atmospheric composition. Its tenuous atmosphere is known to have a substantial sodium component. A Fabry-Perot etalon is used to achieve a compact design. A one degree-of-freedom 
Fig. 31 MSASI: total instrument mass $\tilde{3} .5 \mathrm{~kg}$; power consumption $\tilde{1} 5.2$ (average) W

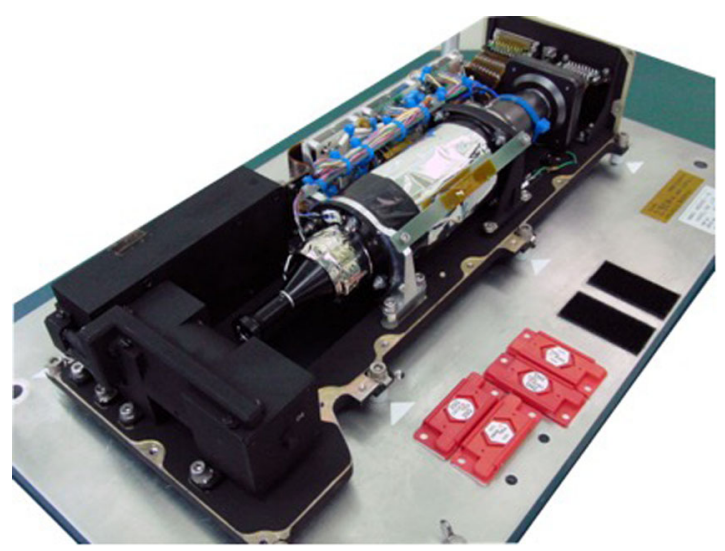

scanning mirror is employed to obtain full-disk images of the planet. The radiation-tolerant Complementary Metal Oxide Semiconductor (CMOS) device with an image intensifier is used as a photon detector. The Fabry-Perot interferometer comprises two parallel, flat, transparent plates coated with a film of high reflectivity. Its principal advantage is that its throughput is much higher than that of a prism or grating spectrometer. BepiColombo will use this design for the first time to show its high-spectral performance in planetary sciences. The combination of Fabry-Perot etalon and filter is acceptable, given the mass and power limitations for the instrument (Fig. 31), and will provide the high transmission (60\%) and the spectral resolution $(0.007 \mathrm{~nm})$. The scientific objectives of MSASI include the investigation of the dominant release process for sodium, the measurement of the dusk-dawn asymmetry and the sodium tail, and the study of topographic effects. Source processes for sodium considered likely are thermal desorption, photon-stimulated desorption, sputtering by impacting particles of the solar wind, and meteoroid vaporisation. For more details see Yoshikawa et al. (2010).

PWI (Plasma Wave Investigation, Fig. 32) consists of two sets of electric field sensors (MEFISTO and WPT) (Karlsson et al. 2020) and two kinds of magnetic field sensors (LF-SC and DB-SC), which are connected to three receivers (EWO, SORBET and AM2P) (Yagitani et al. 2020). The PWI will observe both waveforms and frequency spectra in the frequency range from DC to $10 \mathrm{MHz}$ for the electric field and from $0.1 \mathrm{~Hz}$ to $640 \mathrm{kHz}$ for the magnetic field. PWI was designed and developed in collaboration between Japanese and European scientists. It will give important information regarding energy exchange processes in the small magnetosphere where the role of micro-physics is more visible than anywhere else. PWI will allow us to study the activities of electric fields, plasma waves, and radio waves in the Hermean plasma environments through the first in-situ and remote-sensing observations. The global electric field measurement provides information on plasma transport and acceleration in the magnetosphere. The plasma waves instrument provides information on energy and momentum exchange processes governing collisionless plasmas. The radio waves can be used as remote-sensing tools for magnetospheric activities such as Earth-like auroral kilometric radiation, etc. Solar radiation will also be used as a solar activity monitor in the heliocentric sector facing Mercury by stereoscopic studies combined with similar measurements in Earth orbit. The PWI also has the capability to measure the electron density and temperature as fundamental plasma diagnostics. Through collaboration with other insitu and remote-sensing measurements aboard the BepiColombo's MPO and Mio, these will give important information on the structure, dynamics, and physical processes in the unique magnetosphere and exosphere of Mercury. For more details see Kasaba et al. (2020a). 

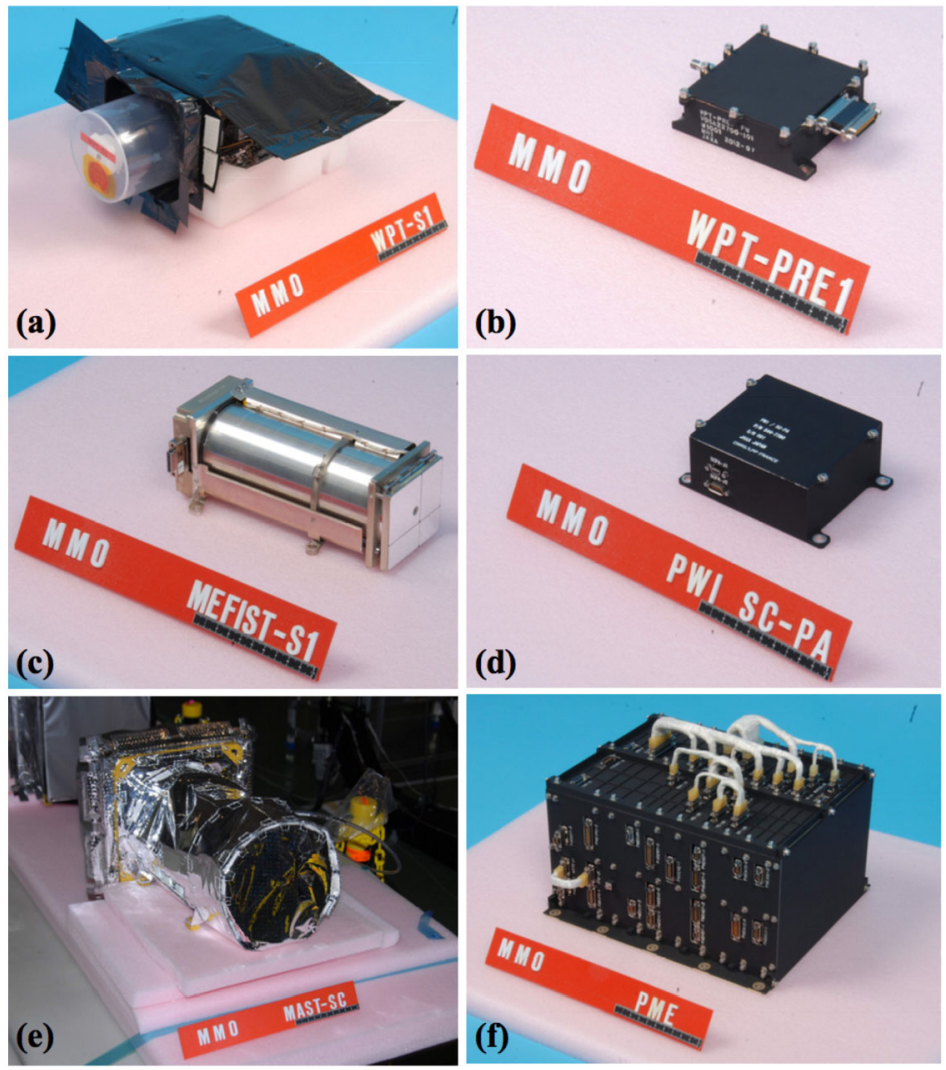

Fig. 32 PWI instrument suite: (a) WPT-S. (b) WPT-Preamp. (c) MEFISTO-S. (d) SC-Preamp. (e) MASTSC with SCM. (f) PME; total mass $\tilde{1} 6.0 \mathrm{~kg}$; power consumption $\tilde{8} .8$ (average) W acronyms explained in the text; images taken from Kasaba et al. (2020a)

Fig. 33 BERM: total mass $\tilde{2} .0 \mathrm{~kg}$; power consumption 4.9 W

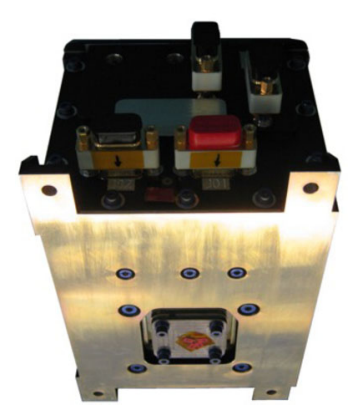

\subsection{Other instruments}

The BepiColombo Radiation Monitor (BERM, Fig. 33) is integrated in the radiator panel of the MPO and is thus exposed to the -Y direction. The detector is made of a stack of silicon detectors that are controlled by floating point gate array (FPGA) responsible for the individual particle recognition process, the communications to the MPO on-board computer, 
Fig. 34 CAM electronic box (mass $0.93 \mathrm{~kg}$ ) and the three MCAMs (mass $\tilde{0} .11 \mathrm{~kg}$; power consumption $\tilde{1} .5$ (each camera) W

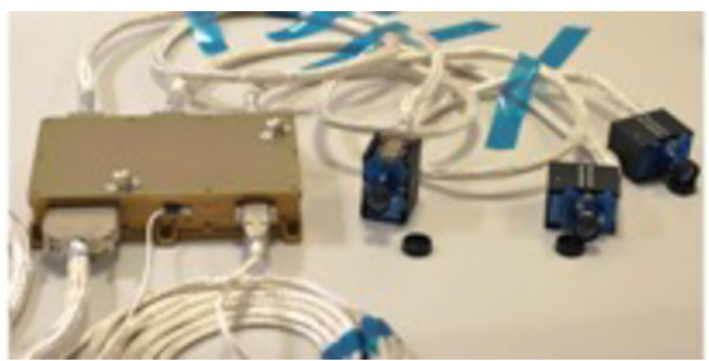

Fig. 35 MCAM 1 and 2 (left) in compartment of -X panel, MCAM 3 in compartment of $+X$ panel
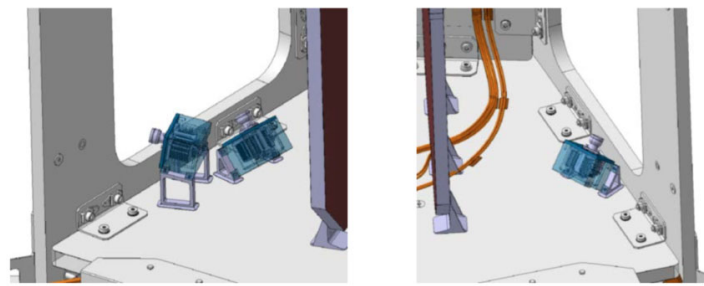

the provision of housekeeping information, and the scrubbing of the internal memory. The detector samples every 30 seconds with a field-of-view of $40^{\circ}$. The individual detectors have a thickness of $300 \mu \mathrm{m}$, with the exception of the top-detector having a thickness of $200 \mu \mathrm{m}$. The gain of the individual detectors can be controlled by FPGA settings, which allows the detailed calibration of the overall detector stack towards the expected incoming particle (electrons, protons, heavy ions) energy. The expected performance of BERM is $1-20 \mathrm{MeV}$ for protons, $0.3-10 \mathrm{MeV}$ for electrons, and $1-50 \mathrm{MeV}$ for heavy ions. The instrument processes the counts internally into energy bins ( 5,8 and 5 for electrons, protons and heavy ions, respectively).

The MTM houses three BepiColombo Monitoring Cameras (MCAMs, Fig. 34) located in three different compartments: one located on the MTM $+X$ panel and two on the MTM -X panel (Fig. 35). The individual cameras are mounted such that MCAM1 images the solar panel in -X direction, MCAM2 the Medium-Gain Antenna (MGA) and the MPO-MAG, and MCAM3 the High-Gain Antenna (HGA) Fig. 36. The cameras are identical, off-theshelf micro-cameras, based on a $1 k \times 1 k \mathrm{CCD}, 10$-bit pixel resolution, and a $10 \mathrm{Mbit} / \mathrm{s}$ camera transfer speed. The cameras have a diagonal field-of-view of 70 degrees. The optics comprise a $14 \mathrm{~mm}$ front lens, a $12.385 \mathrm{~mm}$ focal length, an entrance pupil diameter of $1.239 \mathrm{~mm}$, an F-number of 10, which results in an optical transmittance of larger than $45 \%$. The spectral range covers $480 \mathrm{~nm}$ to $940 \mathrm{~nm}$. All cameras are controlled by a single control board (CAM box) that can hold a maximum of 64 images, before being transferred to the MPO Solid State Mass Memory. The cameras can be operated in an automatic mode, as well as manually commanded to set the integration time, the gain, and image mode (full or half window).

\section{Conclusion}

BepiColombo is the planetary cornerstone of ESA's Cosmic Vision Programme and is devoted to the thorough exploration of Mercury and its environment. It is being carried out as a joint project between ESA and JAXA. With its two-spacecraft, interdisciplinary approach, 

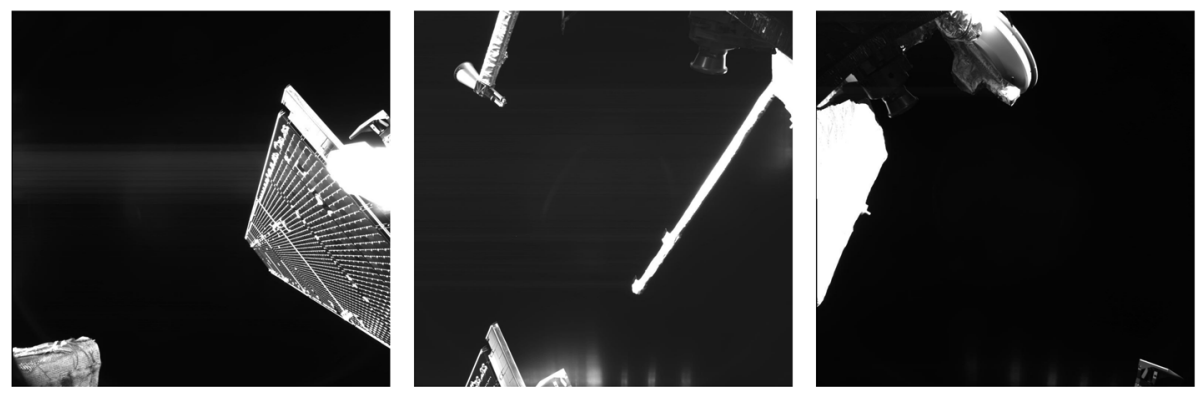

Fig. 36 MCAM1 view on solar panel (left); MCAM2 view on MGA and MPO-MAG (center); MCAM3 image on HGA (right

the BepiColombo mission will provide the detailed information necessary to understand the process of planetary formation and evolution in the hottest part of the proto-planetary nebula, as well as the similarities and differences between the magnetospheres of Mercury and Earth. To accomplish this, a global characterization of Mercury is required, which will be achieved with a thorough investigation of its interior, surface, exosphere, and magnetosphere. In addition, the mission offers unique possibilities for testing Einstein's theory of general relativity. The understanding of the formation and present status of terrestrial planets needs to be improved significantly in order to understand how terrestrial planets could form and evolve in other solar systems ((McNutt et al. 2004) and (Balogh et al. 2007)).

BepiColombo comprises 3 complete spacecraft and a sunshield (see Fig. 2) flying together as a mechanically and electrically coupled stack from launch up until the Mercury orbit insertions of its two orbiters. The two scientific orbiters, MPO and Mio, are brought to Mercury by the Mercury Transfer Module (MTM), a large propulsion module and the primary source of electric power for the 7.2-year-long cruise to Mercury. The high delta-V required to get into Mercury orbit and the total mass to be carried posed challenging requirements to the mission analysis and to the propulsion systems. The selected strategy is to make use of multiple planetary gravity assists to generate the bulk of the required delta- $\mathrm{V}$ together with high-power electric propulsion to generate the remaining velocity increment and to ensure the correct trajectory between flybys. If required, small chemical bi-propellant burns are employed to perform Trajectory Correction Maneuvers (TCMs) before and after the flybys, based on tracking data from ground. The MTM hosts the four SEP thrusters and provides all of the electric power for the 3 modules during cruise. The MTM will bring the two Mercury orbiting modules into a position from which they can reach their final science orbits around Mercury. The sunshield is needed to protect the Mio spacecraft from overheating during the cruise and will be jettisoned after delivering Mio into its target science orbit. When BepiColombo's two orbiters start operation in their polar orbits around Mercury in early 2026 our knowledge about Mercury will be enhanced by results of the measurements from 16 instruments, which ensure a comprehensive exploration of the planet and its environment.

While looking ahead to what results we could expect from BepiColombo's MPO and Mio in orbit, one could also think about the next step in Mercury explorations in the years to come (McNutt et al. 2018). Thanks to Mariner 10 and MESSENGER we already know that Mercury is a mysterious planet, which still has not disclosed all its secrets one needs to know in order to better understand the evolution of our planetary system. BepiColombo with its two spacecraft and its state-of-the-art instrumentation is perfectly suited to reach the 
next step in order to gain a better knowledge of Mercury. However, after the comprehensive remote sensing, internal field and environment investigations performed by BepiColombo, a lander mission would provide very useful complementary information on the surface of Mercury. It would also provide ground truth on the elemental and mineralogical composition of a specific site which would be very valuable as ground calibration enhancing the value of the BepiColombo remote sensing data. After a flyby and two orbiter missions the next fundamental step in explorations could be a lander mission (Hauck et al. 2010). The last Planetary Missions Concept Studies Report (Ernst et al. 2020) concluded that landing on Mercury is technically feasible with a state of the art payload and financially affordable.

In the other articles of this special issue the science objectives, measurements requirements, and the design and first commissioning results of each instrument on both MPO and Mio are described. In addition, some overview articles on surface and composition, geophysical and geochemical science goals, the Hermean environment, the science plans during the cruise phase and fly-bys, and the BepiColombo ground segment will make this issue a comprehensive source of information on what the scientists expect from the BepiColombo mission en route and upon its arrival at Mercury.

Acknowledgements The authors wish to thank the entire Project Team of industry especially Airbus Defence and Space GmbH (Airbus-DE, TAS-I and Airbus-UK), the Project Team at JAXA, and the Project Team at ESA, the Operations Team at ESOC and the Science Operations Team at ESAC for all their hard work to make this mission possible and for their contributions to the system definition and for providing the information for this article. The authors would also like to thank the two reviewers for their detailed, thorough review and for many helpful comments and suggestions to improve the quality of the paper. Special thanks to Jan van Casteren, BepiColombo Project Manager, who passed away much to early.

Open Access This article is licensed under a Creative Commons Attribution 4.0 International License, which permits use, sharing, adaptation, distribution and reproduction in any medium or format, as long as you give appropriate credit to the original author(s) and the source, provide a link to the Creative Commons licence, and indicate if changes were made. The images or other third party material in this article are included in the article's Creative Commons licence, unless indicated otherwise in a credit line to the material. If material is not included in the article's Creative Commons licence and your intended use is not permitted by statutory regulation or exceeds the permitted use, you will need to obtain permission directly from the copyright holder. To view a copy of this licence, visit http://creativecommons.org/licenses/by/4.0/.

\section{References}

B.J. Anderson, C.L. Johnson, H. Korth, J.A. Slavin, R.M. Winslow, R.J. Phillips, R.L. McNutt Jr., S.C. Solomon, Steady-state field-aligned currents at Mercury. Geophys. Res. Lett. 41(21), 7413-7761 (2014). https://doi.org/10.1002/2014GL061677. pp. i-v

D.J. Anderson, G. Colombo, P.B. Esposito, E.L. Lau, G.B. Trager, The mass, gravity field, and ephemeris of Mercury. Icarus 71, 337-349 (1987). https://doi.org/10.1016/0019-1035(87)90033-9

J.D. Anderson, J.K. Campbell, J.E. Ekelund, J. Ellis, J.F. Jordan, Anomalous orbital-energy changes observed during spacecraft flybys of Earth. Phys. Rev. Lett. 100, 091102 (2008). https://doi.org/10.1103/ PhysRevLett.100.091102

B.J. Anderson, M.H. Acun a, H. Korth, J.A. Slavin, H. Uno, C.L. Johnson, M.E. Purucker, S.C. Solomon, J.M. Raines, T.H. Zurbuchen, G. Gloecker, R.L. Mc-Nutt Jr., The magnetic field of Mercury. Space Sci. Rev. 152, 307-339 (2010). https://doi.org/10.1007/s11214-009-9544-3

A.M. Archibald, N.V. Gusinskaia, J.W.T. Hessels, A.T. Deller, D.L. Kaplan, D.R. Lorimer, R.S. Lynch, S.M. Ransom, I.H. Stairs, Universality of free fall from the orbital motion of a pulsar in a stellar triple system. Nature 559, 73-76 (2018). https://doi.org/10.1038/s41586-018-0265-1

Balogh, A. (Coordinator), Mercury Orbiter, a mission proposal to ESA to be considered as a candidate for the M3 mission, 1993. ESA, Presentation of Assessment Study Results, European Space Agency, SC1 (94)9 (1994)

A. Balogh, in The BepiColombo Mission to Mercury a Brief History, EGU Annual Assembly, Vienna, April 2005 (2005), 
A. Balogh, R. Grard, G. Scoon, M. Hechler, A Mercury orbiter mission: report on the European Space Agency's assessment study, in Abstracts of the 25th Lunar and Planetary Science Conference, 14-18 March 1994, Houston, TX (1994), p. 49

A. Balogh, R. Grard, S.C. Solomon, R. Schulz, Y. Langevin, Y. Kasaba, M. Fujimoto, Missions to Mercury. Space Sci. Rev. 132, 611-645 (2007)

W. Baumjohann, A. Matsuoka, Y. Narita, W. Magnes, D. Heyner, K.-H. Glassmeier, R. Nakamura, D. Fischer, F. Plaschke, M. Volwerk, T.L. Zhang, H.-U. Auster, I. Richter, A. Balogh, C. Carr, M. Dougherty, T.S. Horbury, H. Tsunakawa, M. Matsushima, M. Shinohara, H. Shibuya, T. Nakagawa, M. Hoshino, Y. Tanaka, B.J. Anderson, C.T. Russell, U. Motschmann, F. Takahashi, A. Fujimoto, The BepiColomboMio magnetometer en route to Mercury. Space Sci. Rev. 216 (2020), this journal. https://doi.org/10. 1007/s11214-020-00745-z

J. Beerer, Historical account of return trajectory. Interoffice Memorandum, Beerer to Gordon, 16 July 1970. Jet Propulsion Laboratory, Pasadena (1970), 1 p.

J.W. Belcher, J.A. Slavin, T.P. Armstrong, R.W. Farquhar, S.-I. Akasofu, D.N. Baker, C.A. Cattell, A.F. Cheng, E.L. Chupp, P.E. Clark, M.E. Davies, E.W. Hones, W.S. Kurth, J.K. Maezawa, F. Mariani, E. Marsch, G.K. Parks, E.G. Shelley, G.L. Siscoe, E.J. Smith, R.G. Strom, J.I. Trombka, D.J. Williams, C.W. Yen, Mercury Orbiter: report of the Science Working Team. Technical Memorandum 4255, NASA, Washington, D. C. (1991), p. 132

J. Benkhoff, J. van Casteren, H. Hayakawa, M. Fujimoto, H. Laakso, BepiColombo-Comprehensive Exploration of Mercury: Mission Overview and Science Goals (2010)

W. Benz, W.L. Slattery, A.G.W. Cameron, Collisional stripping of Mercury's mantle. Icarus 74, 516-528 (1988)

D.T. Blewett (2018). https://doi.org/10.1017/9781316650684.013

D.T. Blewett et al., Hollows on Mercury: evidence for geologically recent volatile-related activity. Science 333, 1,856-1,859 (2011). https://doi.org/10.1126/science.1211681

D.T. Blewett, W.M. Vaughan, Z. Xiao, N.L. Chabot, B.W. Denevi, C.M. Ernst, J. Helbert, M. D’Amore, A. Maturilli, J.W. Head, S.C. Solomon, Mercury's hollows: constraints on formation and composition from analysis of geological setting and spectral reflectance. J. Geophys. Res., Planets 118(5), 1013-1032 (2013). https://doi.org/10.1029/2012JE004174

D.T. Blewett et al., Analysis of MESSENGER high-resolution images of Mercury's hollows and implications for hollow formation. J. Geophys. Res., Planets 121, 1,798-1,813 (2016). https://doi.org/10.1002/ 2016JE005070

E.J. Bunce, A. Martindale, S. Lindsay, K. Muinonen, D.A. Rothery, J. Pearson, I. McDonnell, C. Thomas, J. Thornhill, T. Tikkanen, C. Feldman, J. Huovelin, S. Korpela, E. Esko, A. Lehtolainen, J. Treis, P. Majewski, M. Hilchenbach, T. Väisänen, A. Luttinen, T. Kohout, A. Penttilä, J. Bridges, K.H. Joy, M. Angeles Alcacera-Gil, G. Alibert, M. Anand, N. Bannister, C. Barcelo-Garcia, C. Bicknell, O. Blake, P. Bland, G. Butcher, A. Cheney, U. Christensen, T. Crawford, I. Crawford, K. Dennerl, M. Dougherty, P. Drumm, R. Fairbend, M. Genzer, M. Grande, G.P. Hall, R. Hodnett, P. Houghton, S. Imber, E. Kallio, M.L. Lara, A. Balado Margeli, J. Miguel Mas-Hesse, S. Maurice, S. Milan, P. Millington-Hotze, S. Nenonen, L. Nittler, T. Okada, J. Ormör, J. Perez-Mercader, R. Poyner, E. Robert, D. Ross, M. Pajas Sanz, E. Schyns, J. Seguy, L. Strüder, N. Vaudon, J. Viceira-Martín, H. Williams, D. Willingale, T. Yeoman, The BepiColombo Mercury Imaging X-ray Spectrometer: science goals, instrument performance and operations. Space Sci. Rev. 216(8), 1-38 (2020). https://doi.org/10.1007/s11214-020-00750-2

P.K. Byrne et al., Mercury's global contraction much greater than earlier estimates. Nat. Geosci. 7, 301-307 (2014). https://doi.org/10.1038/ngeo2097

N.L. Chabot, C.M. Ernst, B.W. Denevi, H. Nair, A.N. Deutsch, D.T. Blewett, S.L. Murchie, A.N. Deutsch, J.W. Head, S.S. Solomon, Imaging Mercury's polar deposits during MESSENGER's low-altitude campaign. Geophys. Res. Lett. 43(18), 9461-9468 (2016)

N.L. Chabot, E.E. Shread, J.K. Harmon, Investigating Mercury's south polar deposits: Arecibo radar observations and high-resolution determination of illumination conditions. J. Geophys. Res., Planets 123, 666-681 (2018). https://doi.org/10.1002/2017JE005500

G. Colombo, Rotational period of the planet Mercury. Nature 208, 575 (1965). https://doi.org/10.1038/ $208575 \mathrm{a} 0$

G. Colombo, I.I. Shapiro, The rotation of the planet Mercury. Astrophys. J. 145, 295-307 (1966). https://doi. org/10.1086/148762

A. Coradini, F. Capaccioni, P. Drossart, A. Semery, G. Arnold, U. Schade, F. Angrilli, M.A. Barucci, G. Bellucci, G. Bianchini, J.P. Bibring, A. Blanco, M. Blecka, D. Bockelee-Morvan, R. Bonsignori, M. Bouye, E. Bussoletti, M.T. Capria, R. Carlson, U. Carsenty, P. Cerroni, L. Colangeli, M. Combes, M. Combi, J. Crovisier, M. Dami, M.C. DeSanctis, A.M. DiLellis, E. Dotto, T. Encrenaz, E. Epifani, S. Erard, S. Espinasse, A. Fave, C. Federico, U. Fink, S. Fonti, V. Formisano, Y. Hello, H. Hirsch, G. Huntzinger, R. Knoll, D. Kouach, W.H. Ip, P. Irwin, J. Kachlicki, Y. Langevin, G. Magni, T. McCord, 
V. Mennella, H. Michaelis, G. Mondello, S. Mottola, G. Neukum, V. Orofino, R. Orosei, P. Palumbo, G. Peter, B. Pforte, G. Piccioni, J.M. Reess, E. Ress, B. Saggin, B. Schmitt, D. Stefanovitch, A. Stern, F. Taylor, D. Tiphene, G. Tozzi, Virtis: an imaging spectrometer for the Rosetta mission. Planet. Space Sci. 46(9-10), 1291-1304 (1998). https://doi.org/10.1016/S0032-0633(98)00025-7

A.C.M. Correia, J. Laskar, Mercury's capture into the $3 / 2$ spin-orbit resonance as a result of its chaotic dynamics. Nature 429, 848-850 (2004)

G. Cremonese, F. Capaccioni, M.T. Capria, A. Doressoundiram, P. Palumbo, M. Vincendon, M. Massironi, S. Debei, M. Zusi, F. Altieri, M. Amoroso, G. Aroldi, M. Baroni, A. Barucci, G. Bellucci, J. Benkhoff, S. Besse, C. Bettanini, M. Blecka, D. Borrelli, J.R. Brucato, C. Carli, P. Cerroni, A. Cicchetti, L. Colangeli, M. Dami, V. Da Deppo, V. Della Corte, M.C. De Sanctis, S. Erard, F. Esposito, D. Fantinel, L. Ferranti, F. Ferri, I. Ficai Veltroni, G. Filacchione, E. Flamini, G. Forlani, S. Fornasier, O. Forni, M. Fulchignoni, V. Galluzzi, K. Gwinner, W. Ip, L. Jorda, Y. Langevin, L. Lara, F. Leblanc, C. Leyrat, Y. Li, S. Marchi, L. Marinangeli, F. Marzari, E. Mazzotta Epifani, M. Mendillo, V. Mennella, R. Mugnuolo, K. Muinonen, G. Naletto, R. Noschese, E. Palomba, R. Paolinetti, D. Perna, G. Piccioni, R. Politi, F. Poulet, R. Ragazzoni, C. Re, M. Rossi, A. Rotundi, G. Salemi, M. Sgavetti, E. Simioni, N. Thomas, L. Tommasi, A. Turella, T. Van Hoolst, L. Wilson, F. Zambon, A. Aboudan, O. Barraud, N. Bott, P. Borin, G. Colombatti, M. El Yazidi, S. Ferrari, J. Flahault, L. Giacomini, L. Guzzetta, A. Lucchetti, E. Martellato, M. Pajola, A. Slemer, G. Tognon, D. Turrini, SIMBIO-SYS: cameras and spectrometer for the BepiColombo mission. Space Sci. Rev. 216(5), 1-78 (2020). https://doi.org/10.1007/s11214-02000704-8

C. Damiani, J.-P. Rozelot, S. Lefebvre, A. Kilcik, A.G. Kosovichev, A brief history of the solar oblateness. A review. J. Atmos. Sol.-Terr. Phys. 73(2-3), 241-250 (2011). https://doi.org/10.1016/j.jastp.2010.02. 021. hal-00519433 (2010)

F. De Marchi, G. Cascioli, Testing general relativity in the solar system: present and future perspectives. Class. Quantum Gravity 37, 095007 (2020). https://doi.org/10.1088/1361-6382/ab6ae0

M.L. Delitsky, D.A. Paige, M.A. Siegler, E.R. Harju, D. Schriver, R.E. Johnson, P. Travnicek, Ices on Mercury: chemistry of volatiles in permanently cold areas of Mercury's north polar region. Icarus 281, 19-31 (2017)

G.A. Di Braccio, J.A. Slavin, S.A. Boardsen, B.J. Anderson, H. Korth, T.H. Zurbuchen, J.M. Raines, D.N. Baker, R.L. McNutt, S.C. Solomon, MESSENGER observations of magnetopause structure and dynamics at Mercury. J. Geophys. Res. Space Phys. 118, 997-1008 (2013)

I. Di Stefano, P. Cappuccio, L. Iess, The BepiColombo solar conjunction experiments revisited. Class. Quantum Gravity 38 (2021). https://doi.org/10.1088/1361-6382/abd301

D.L. Domingue, C.R. Chapman, R.M. Killen, T.H. Zurbuchen, J.A. Gilbert, M. Sarantos Mehdi Benna, J.A. Slavin, D. Schriver, P.M. Travnicek, T.M. Orlando, A.L. Sprague, D.T. Blewett, J.J. Gillis-Davis, W.C. Feldman, D.J. Lawrence, G.C. Ho Denton, S. Ebel, L.R. Nittler, F. Vilas Carle, M. Pieters, S.C. Solomon, C.L. Johnson, R.M. Winslow, J. Helbert, P.N. Peplowski, S.Z. Weider, N. Mouawad, N.R. Izenberg, W.E. McClintock, Mercury's weather-beaten surface: understanding Mercury in the context of lunar and asteroidal space weathering studies. Space Sci. Rev. 181, 121-214 (2014). https://doi.org/ 10.1007/s11214-014-0039-5

A. Einstein, Die Grundlage der allgemeinen Relativitätstheorie. Ann. Phys. 354, 769-822 (1916)

C. Ernst et al., Mercury Lander - Transformative science from the surface of the innermost planet, Planetary mission concept study for the 2023-2032 decadal survey (2020). https://science.nasa.gov/science-red/ s3fs-public/atoms/files/Mercury

A. Ferrero, D. Battaglia, T. Malosti, D. Stramaccioni, J. Schilke, The challenges of the thermal design of BepiColombo Mercury planet orbiter, in 46th International Conference on Environmental Systems, ICES-2016-212, Vienna, Austria (2016)

B.H. Foing, G.D. Racca, A. Marini, E. Evrard, L. Stagnaro, M. Almeida, D. Koschny, D. Frew, J. Zender, J. Heather, M. Grande, J. Huovelin, H.U. Keller, A. Nathues, J.L. Josset, A. Malkki, W. Schmidt, G. Noci, R. Birkl, L. Iess, Z. Sodnik, P. McManamon, SMART-1 mission to the Moon: status, first results and goals. Adv. Space Res. 37(1), 6-13 (2006). https://doi.org/10.1016/j.asr.2005.12.016

N.J. Fox, M.C. Velli, S.D. Bale, R. Decker, A. Driesman, R.A. Howard, J.C. Kasper, J. Kinnison, M. Kusterer, D. Lario, M.K. Lockwood, D.J. McComas, N.E. Raouafi, A. Szabo, The Solar Probe Plus mission: humanity's first visit to our star. Space Sci. Rev. 204(1-4), 7-48 (2016). https://doi.org/10.1007/s11214015-0211-6

A. Genova et al., Geodetic evidence that Mercury has a solid inner core. Geophys. Res. Lett. 46(7), 3625-3633 (2019). https://doi.org/10.1029/2018gl081135

A. Genova, H. Hussmann, T. Van Hoolst, D. Heyner, L. Iess, F. Santoli, N. Thomas, P. Cappuccio, I. di Stefano, P. Kolhey, B. Langlais, J.Z.D. Mieth, J.S. Oliveira, A. Stark, G. Steinbrügge, N. Tosi, J. Wicht, J. Benkhoff, Geodesy, geophysics and fundamental physics investigations of the BepiColombo mission. Space Sci. Rev. 217, 31 (2021). https://doi.org/10.1007/s11214-021-00808-9 
R. Grard, The Mercury Orbiter mission. Adv. Space Res. 19(IO), 1621-1624 (1997)

R. Grard, A. Balogh, Returns to Mercury: science and mission objectives. Planet. Space Sci. 49, 1395-1407 (2001)

H. Gray, J. Bolter, K. Kempkens, P. Randall, N. Wallace, BepiColombo - the Mercury transfer module, in The 36th International Electric Propulsion Conference, University of Vienna, Austria, September 15-20 (2019)

E. Grün, H. Fechtig, P. Gammelin, J. Kissel, The dust experiment onboard Helios (E 10) [Das Staubexperiment auf HELIOS (E10)]. Raumfahrtforschung 19, 268-269 (1975)

J.K. Harmon, M.A. Slade, Radar mapping of Mercury: full-disk images and polar anomalies. Science 258, 640-643 (1992)

J.K. Harmon, M.A. Slade, R.A. Vélez, A. Crespo, M.J. Dryer, J.M. Johnson, Radar mapping of Mercury's polar anomalies. Nature 369, 213-215 (1994)

S.A. Hauck II, D.A. Eng, A. Treiman, G. Tahu, K. Lindstrom, D. Blewett, H. Seifert, K. Stambaugh, G. Chavers, S. Oleson, M. Mcguire, Y. Guo, J. Dankanich, C. Dong, L. Burke, H.M. LarryWolfarth, J. Drexler, M. Holdridge, J. Cockrell, T. Miller, H. Trinh, J. Fittje, T. Verhey, J. Gyekenyesi, J. Ercol, E. Abel, T. Colozza, B. Sequeira, J. Warner, M. Fraeman, G. Williams, P. Schmitz, E. Lowery, G. Landis, J. Hojniki, D. Adams, M. Martini, S. Williams, J. Drexler, Mercury Lander Mission Concept Study (The Johns Hopkins University Applied Physics Laboratory, Laurel, 2010). 132 pp.

J.W. Head, S.L. Murchie, L.M. Prockter, M.S. Robinson, S.C. Solomon, R.G. Strom, C.R. Chapman, T.R. Watters, W.E. McClintock, D.T. Blewett, J.J. Gillis-Davis, Volcanism on Mercury: evidence from the first MESSENGER flyby. Science 321, 69-72 (2008)

D. Heyner et al., The fluxgate magnetometer of the BepiColombo Mercury Planetary Orbiter. Space Sci. Rev. 217(4) (2021). https://doi.org/10.1007/s11214-021-00822-X

H. Hiesinger, J. Helbert, G. Alemano, K.E. Bauch, M. D’Amore, A. Maturilli, A. Morlok, M.P. Reitze, C. Stangarone, A.N. Stojic, I. Varatharajan, I. Weber (the MERTIS Co-I Team), Correction to: Studying the composition and mineralogy of the hermean surface with the Mercury Radiometer and Thermal Infrared Spectrometer (MERTIS) for the BepiColombo mission: an update. Space Sci. Rev. 216(8), 1 (2020). https://doi.org/10.1007/s11214-020-00780-w

J. Huovelin, R. Vainio, E. Kilpua, A. Lehtolainen, S. Korpela, E. Esko, K. Muinonen, E. Bunce, A. Martindale, M. Grande, H. Andersson, S. Nenonen, J. Lehti, W. Schmidt, M. Genzer, T. Vihavainen, J. Saari, J. Peltonen, E. Valtonen, M. Talvioja, P. Portin, S. Narendranath, R. Jarvinen, T. Okada, A. Milillo, M. Laurenza, E. Heino, P. Oleynik, Solar intensity X-ray and particle spectrometer SIXS: instrument design and first results. Space Sci. Rev. 216(5), 1-42 (2020). https://doi.org/10.1007/s11214-020-00717-3

K. Hurley et al., The InterPlanetary network supplement to the second Fermi GBM catalog of cosmic gammaray bursts. Astrophys. J. Suppl. Ser. 229(2), 31 (2017)

L. Iess, S.W. Asmar, P. Cappuccio, G. Cascioli, F. De Marchi, I. di Stefano, A. Genova, N. Ashby, J.P. Barriot, P. Bender, C. Benedetto, J.S. Border, F. Budnik, S. Ciarcia, T. Damour, V. Dehant, G. Di Achille, A. Di Ruscio, A. Fienga, R. Formaro, S. Klioner, A. Konopliv, A. Lemaître, F. Longo, M. Mercolino, G. Mitri, V. Notaro, A. Olivieri, M. Paik, A. Palli, G. Schettino, D. Serra, L. Simone, G. Tommei, P. Tortora, T. Van Hoolst, D. Vokrouhlický, M. Watkins, X. Wu, M. Zannoni, Gravity, geodesy and fundamental physics with BepiColombo's MORE investigation. Space Sci. Rev. 217, 21 (2021). https://doi.org/10. 1007/s11214-021-00800-3

L. Imperi, L. Iess, The determination of the post-Newtonian parameter $\gamma$ during the cruise phase of BepiColombo. Class. Quantum Gravity 34(7), 075002 (2017)

N.R. Izenberg, R.L. Klima, S.L. Murchie, D.T. Blewett, G.M. Holsclaw, W.E. McClintock, E. Malaret, C. Mauceri, F. Vilas, A.L. Sprague, J. Helbert, D.L. Domingue, J.W. Head III, T.A. Goudge, S.C. Solomon, C.A. Hibbitts, M. Darby Dyar, The low-iron, reduced surface of Mercury as seen in spectral reflectance by MESSENGER. Icarus 228, 364-374 (2014)

R. Jehn, S. Campagnola, D. Garcia, S. Kemble, Low-thrust approach and gravitational capture at Mercury, in 18th Int. Symposium on Space Flight Dynamics, Munich, Germany, 11-15 October (2004)

X. Jia, J.A. Slavin, T.I. Gombosi, L.K.S. Daldorff, G. Toth, B. Holst, Global MHD simulations of Mercury's magnetosphere with coupled planetary interior: induction effect of the planetary conducting core on the global interaction. J. Geophys. Res. Space Phys. 120, 4763-4775 (2015)

T. Karlsson, Y. Kasaba, J.-E. Wahlund, P. Henri, L. Bylander, W. Puccio, S.-E. Jansson, L. Åhlen, E. Kallio, H. Kojima, A. Kumamoto, K. Lappalainen, B. Lybekk, K. Ishisaka, A. Eriksson, M. Morooka, The MEFISTO and WPT electric field sensors of the Plasma Wave Investigation on the BepiColombo Mio spacecraft - measurements of low and high frequency electric fields at Mercury. Space Sci. Rev. 216(8), 1-24 (2020). https://doi.org/10.1007/s11214-020-00760-0

Y. Kasaba, H. Kojima, M. Moncuquet, J.-E. Wahlund, S. Yagitani, F. Sahraoui, P. Henri, T. Karlsson, Y. Kasahara, A. Kumamoto, K. Ishisaka, K. Issautier, G. Wattieaux, T. Imachi, S. Matsuda, J. Lichtenberger, H. Usui, Plasma Wave Investigation (PWI) aboard BepiColombo Mio on the trip to the first measurement 
of electric fields, electromagnetic waves, and radio waves around Mercury. Space Sci. Rev. 216(4), 1-54 (2020a). https://doi.org/10.1007/s11214-020-00692-9

Y. Kasaba, T. Takashima, S. Matsuda, S. Eguchi, M. Endo, T. Miyabara, M. Taeda, Y. Kuroda, Y. Kasahara, T. Imachi, H. Kojima, S. Yagitani, M. Moncuquet, J.-E. Wahlund, A. Kumamoto, A. Matsuoka, W. Baumjohann, S. Yokota, K. Asamura, Y. Saito, D. Delcourt, M. Hirahara, S. Barabash, N. Andre, M. Kobayashi, I. Yoshikawa, G. Murakami, H. Hayakawa, Mission data processor aboard the BepiColombo Mio spacecraft: design and scientific operation concept. Space Sci. Rev. 216(3), 1-19 (2020b). https:// doi.org/10.1007/s11214-020-00658-x

K. Kempkens, F. Striedter, H. Gray, S. Clark, K. Chan, N. Wallace, BepiColombo - the Mercury transfer module, in The 36th International Electric Propulsion Conference, University of Vienna, Austria September 15-20 (2019)

R.M. Killen, J.M. Hahn, Impact vaporization as a possible source of Mercury's calcium exosphere. Icarus 250, 230-237 (2015)

R.M. Killen, J. Benkhoff, T.H. Morgan, Mercury's polar caps and the generation of an $\mathrm{OH}$ exosphere. Icarus 125, 195-211 (1997)

R.M. Killen, G. Cremonese, H. Lammer, S. Orsini, A.E. Potter, A.L. Sprague, P. Wurz, M.L. Khodachenko, H.I.M. Lichtenegger, A. Milillo, A.R. Mura, Processes that promote and deplete the exosphere of Mercury. Space Sci. Rev. 132, 433-509 (2007)

M. Kobayashi, K. Nogami, M. Fujii, H. Ohashi, T. Miyachi, S. Sasaki, S. Hasegawa, H. Yano, H. Shibata, T. Iwai, S. Minami, S. Takechi, E. Grun, R. Srama, Development of the Mercury Dust Monitor (MDM) on board BepiColombo mission. Space Sci. Rev. 216, 144K (2020). https://doi.org/10.1007/s11214-02000775-7

A.S. Kozyrev et al., Selection of new innovation crystal for Mercury Gamma-ray and Neutron Spectrometer on-board MPO/BepiColombo mission, in EGU General Assembly 2015, 12-17 April, 2015, Vienna, Austria (2015), id. 6848

A. Kozyrev, I. Mitrofanov, J. Benkhoff et al., Next generation of scintillation detector based on cerium bromide crystal for space application in the gamma-ray spectrometer of the Mercurian Gamma-ray and Neutron Spectrometer. Instrum. Exp. Tech. 59(4), 569-577 (2016a)

A. Kozyrev et al., A comparative study of gamma-ray spectrometers with $\mathrm{LaBr} 3(\mathrm{Ce} 3+)$ and $\mathrm{CeBr} 3$ scintillation crystals for planetary remote sensing applications, in EGU General Assembly 2016, Vienna, Austria, 17-22 April, 2016 (2016b), id. EPSC2016-16261

A. Kozyrev, A. Anikin, A. Vostrukhin, et al., Simulation of space experiments for nuclear planetology: measurement of relative intensities of lines of gamma-ray emitted upon thermal-neutron capture by nuclei. Phys. At. Nucl. 81(5), 527-539 (2018)

H. Krüger, E. Grünn, D.P. Hamilton, M. Baguhl, S.F. Dermott, H. Fechtig, B.A. Gustafson, M.S. Hanner, M. Horanyi, J. Kissel, B.A. Lindblad, D. Linkert, G. Linkert, I. Mann, J.A.M. McDon-nell, G.E. Morfill, C. Polanskey, R. Riemann, G.H. Schwehm, R. Srama, H.A. Zook, Three years of Galileo dust data: II. 1993 to 1995. Planet. Space Sci. 47, 85-106 (1999)

H. Krüger, A.V. Krivov, E. Grün, A dust cloud of Ganymede maintained by hypervelocity impacts of interplanetary micrometeoroids. Planet. Space Sci. 48, 1457-1471 (2000)

Y. Langevin, Chemical and solar electric propulsion options for a cornerstone mission to Mercury. Acta Astronaut. 47, 443-452 (2000). https://doi.org/10.1016/S0094-5765(00)00084-9

D. Lawrence, A tale of two poles: toward understanding the presence, distribution, and origin of volatiles at the polar regions of the Moon and Mercury. J. Geophys. Res., Planets 122(1), 21-52 (2017). https://doi. org/10.1002/2016JE005167

R.A. Lewis, J. Pérez Luna, N. Coombs, F. Guarducci, Qualification of the T6 thruster for BepiColombo, in Joint Conference of the 30th International Symposium on Space Technology and Science, 34th International Electric Propulsion Conference, and 6th Nano-satellite Symposium, Hyogo-Kobe, Japan (2015), $10 \mathrm{pp}$.

S.T. Lindsay, M.K. James, E.J. Bunce, S.M. Imber, H. Korth, A. Martindale, T.K. Yeoman, Planet. Space Sci. 125, 72-79 (2016)

V. Mangano, M. Dósa, M. Fränz, A. Milillo, J.S. Oliveira, Y.J.Lee, S. McKenna-Lawlor, D. Grassi, D. Heyner, A.S. Kozyrev, R. Peron, J. Helbert, S. Besse, S. de la Fuente, E. Montagnon, J. Zender, M. Volwerk, J.-Y. Chaufray, J.A. Slavin, H.M.A. Krüger, T. Cornet, K. Iwai, Y. Miyoshi, M. Lucente, S. Massetti, C.A. Schmidt, C. Dong, F. Quarati, T. Hirai, A. Varsani, D. Belyaev, J. Zhong, E.K.J. Kilpua, B.V. Jackson, D. Odstrcil, F. Plaschke, R. Vainio, R. Jarvinen, S.L. Ivanovski, Á. Madár, G. Erdős, C. Plainaki, T. Alberti, S. Aizawa, J. Benkhoff, G. Murakami, E. Quemerais, H. Hiesinger, I.G. Mitrofanov, L. Iess, F. Santoli, S. Orsini, H. Lichtenegger, G. Laky, S. Barabash, R. Moissl, J. Huovelin, Y. Kasaba, Y. Saito, M. Kobayashi, W. Baumjohann, BepiColombo science investigations during cruise and flybys at the Earth, Venus and Mercury. Space Sci. Rev. 217, 23 (2021). https://doi.org/10.1007/s11214-021-00797-9 
S. Marchi et al., Global resurfacing of Mercury 4.0-4.1 billion years ago by heavy bombardment and volcanism. Nature 499, 59-61 (2013). https://doi.org/10.1038/nature12280

J.-L. Margot, S.A. Hauck II, E. Mazarico, S. Padovan, S.J. Peale, Mercury's internal structure, in Mercury: The View After MESSENGER, ed. by S.C. Solomon, L.R. Nittler, B.J. Anderson. Cambridge Planetary Science (2018), pp. 85-113

R.L. McNutt Jr., S.C. Solomon, R.E. Gold, J.C. Leary (the MESSENGER Team), The MESSENGER mission to Mercury: development history and early mission status. Adv. Space Res. 38, 564-571 (2006). https:// doi.org/10.1016/j.asr.2005.05.044

R.L. McNutt, S.C. Solomon, R. Grard, M. Novara, T. Mukai, An international program for Mercury exploration: synergy of MESSENGER and BepiColombo. Adv. Space Res. 33, 2126-2132 (2004)

R.L. McNutt, J. Benkhoff, M. Fujimoto, B.J. Anderson, Future missions: Mercury after MESSENGER, in Mercury: The View After MESSENGER, ed. by S.C. Solomon, L.R. Nittler, B.J. Anderson. Cambridge Planetary Science (2018), pp. 544-569

A. Milillo, M. Fujimoto, G. Murakami, J. Benkhoff, J. Zender, S. Aizawa, M. Dósa, L. Griton, D. Heyner, G. Ho, S.M. Imber, X. Jia, T. Karlsson, R.M. Killen, M. Laurenza, S.T. Lindsay, S. McKenna-Lawlor, A. Mura, J.M. Raines, D.A. Rothery, N. André, W. Baumjohann, A. Berezhnoy, P.A. Bourdin, E.J. Bunce, F. Califano, J. Deca, S. de la Fuente, C. Dong, C. Grava, S. Fatemi, P. Henri, S.L. Ivanovski, B.V. Jackson, M. James, E. Kallio, Y. Kasaba, E. Kilpua, M. Kobayashi, B. Langlais, F. Leblanc, C. Lhotka, V. Mangano, A. Martindale, S. Massetti, A. Masters, M. Morooka, Y. Narita, J.S. Oliveira, D. Odstrcil, S. Orsini, M.G. Pelizzo, C. Plainaki, F. Plaschke, F. Sahraoui, K. Seki, J.A. Slavin, R. Vainio, P. Wurz, S. Barabash, C.M. Carr, D. Delcourt, K.-H. Glassmeier, M.N. Grande, M. Hirahara, J. Huovelin, O. Korablev, H. Kojima, H. Lichtenegger, S. Livi, A. Matsuoka, R. Moissl, M. Moncuquet, K. Muinonen, E. Quémerais, Y. Saito, S. Yagitani, I. Yoshikawa, J.-E. Wahlund, Investigating Mercury's environment with the two-spacecraft BepiColombo mission. Space Sci. Rev. 216(5), 1-78 (2020). https://doi.org/10. 1007/s11214-020-00712-8

I.G. Mitrofanov, A.S. Kozyrev, A. Konovalov, M.L. Litvak, A.A. Malakhov, M.I. Mokrousov, A.B. Sanin, V.I. Tret'ykov, A.V. Vostrukhin, Y.I. Bobrovnitskij, T.M. Tomilina, L. Gurvits, A. Owens, The Mercury Gamma and Neutron Spectrometer (MGNS) on board the Planetary Orbiter of the BepiColombo mission. Planet. Space Sci. 58, 116-124 (2010). https://doi.org/10.1016/j.pss.2009.01.005

I.G. Mitrofanov, A.S. Kozyrev, D.I. Lisov, M.L. Litvak, A.A. Malakhov, M.I. Mokrousov, J. Benkhoff, A. Owens, R. Schulz, F. Quarati, Mercury Gamma-ray and Neutron Spectrometer MGNS for Planetary Orbiter of BepiColombo mission: updates of design and the first measurements in space. Space Sci. Rev. 217 (2021). https://doi.org/10.1007/s11214-021-00842-7

E. Montagnon, F. Budnik, M. Casale, S. de la Fuente, S. Martinez, G. Murakami, M. Ogawa, T. Seki, C. Steiger, M. Yamashita, BepiColombo ground segment and mission operations. Space Sci. Rev. 217, 32 (2021). https://doi.org/10.1007/s11214-021-00805-y

D. Müller, R.G. Marsden, O.C. St. Cyr, H.R. Gilbert, Solar orbiter. Exploring the Sun-heliosphere connection. Sol. Phys. 285(1-2), 25-70 (2013). https://doi.org/10.1007/s11207-012-0085-7

G. Murakami, H. Hayakawa, H. Ogawa, S. Matsuda, T. Seki, Y. Kasaba, Y. Saito, I. Yoshikawa, M. Kobayashi, W. Baumjohann, A. Matsuoka, H. Kojima, S. Yagitani, M. Moncuquet, J.-E. Wahlund, D. Delcourt, M. Hirahara, S. Barabash, O. Korablev, M. Fujimoto, Mio - first comprehensive exploration of Mercury's space environment: mission overview. Space Sci. Rev. 216, 113M (2020). https://doi.org/ 10.1007/s11214-020-00733-3

M. Nakamura, T. Imamura, N. Ishii, T. Abe, T. Satoh, M. Suzuki, M. Ueno, A. Yamazaki, N. Iwagami, S. Watanabe, M. Taguchi, T. Fukuhara, Y. Takahashi, M. Yamada, N. Hoshino, S. Ohtsuki, K. Uemizu, G.L. Hashimoto, M. Takagi, Y. Matsuda, K. Ogohara, N. Sato, Y. Kasaba, T. Kouyama, N. Hirata, R. Nakamura, Y. Yamamoto, N. Okada, T. Horinouchi, M. Yamamoto, Y. Hayashi, Overview of Venus orbiter, Akatsuki. Earth Planets Space 63, 443-457 (2011)

R.M. Nelson, L.J. Horn, J.R. Weiss, W.D. Smythe, Lunar Planet. Sci. 25, 985-986 (1994)

R.M. Nelson et al., Acta Astron., Suppl. 35, 387-396 (2005)

L.R. Nittler, N.L. Chabot, T.L. Grove, P.N. Peplowski, The chemical composition of Mercury, in Mercury: The View After MESSENGER, ed. by S.C. Solomon, L.R. Nittler, B.J. Anderson Cambridge Planetary Science (2018), pp. 30-51

M. Novara, The BepiColombo ESA cornerstone mission to Mercury. Acta Astronaut. 51, 387-395 (2002). https://doi.org/10.1016/S0094-5765(02)00065-6

H. Ogawa, T. Yamazaki, A. Okamoto, BepiColombo Mercury magnetospheric orbiter proto-flight model thermal balance/thermal vacuum test, in 45th International Conference on Environmental Systems, Bellevue, Washington, 12-16 July 2015 (2015)

S. Orsini, S. Livi, H. Lichtenegger, S. Barabash, A. Milillo, E. De Angelis, M. Phillips, G. Laky, M. Wieser, A. Olivieri, C. Plainaki, G. Ho, R.M. Killen, J.A. Slavin, P. Wurz, J.-J. Berthelier, I. Dandouras, E. Kallio, S. McKenna-Lawlor, S. Szalai, K. Torkar, O. Vaisberg, F. Allegrini, I.A. Daglis, C. Dong, C.P. Escoubet, 
S. Fatemi, M. Fränz, S. Ivanovski, N. Krupp, H. Lammer, F. Leblanc, V. Mangano, A. Mura, H. Nilsson, J.M. Raines, R. Rispoli, M. Sarantos, H.T. Smith, K. Szego, A. Aronica, F. Camozzi, A.M. Di Lellis, G. Fremuth, F. Giner, R. Gurnee, J. Hayes, H. Jeszenszky, F. Tominetti, B. Trantham, J. Balaz, W. Baumjohann, D. Brienza, U. Bührke, M.-D. Bush, M. Cantatore, S. Cibella, L. Colasanti, G. Cremonese, L. Cremonesi, M. D’Alessandro, D. Delcourt, M. Delva, M. Desai, M. Fama, M. Ferris, H. Fischer, A. Gaggero, D. Gamborino, P. Garnier, B. Gibson, R. Goldstein, M. Grande, V. Grishin, D. Haggerty, M. Holmström, I. Horvath, K.C. Hsieh, A. Jacques, R.E. Johnson, A. Kazakov, K. Kecskemety, H. Krüger, C. Kürbisch, F. Lazzarotto, F. Leblanc, M. Leichtfried, R. Leoni, A. Loose, D. Maschietti, S. Massetti, F. Mattioli, G. Miller, D. Moissenko, A. Morbidini, R. Noschese, F. Nuccilli, C. Nunez, N. Paschalidis, S. Persyn, D. Piazza, M. Oja, J. Ryno, W. Schmidt, J.A. Scheer, A. Shestakov, S.S. Shuvalov, K. Seki, S. Selci, K. Smith, R. Sordini, J. Svensson, L. Szalai, D. Toublanc, C. Urdiales, A. Varsani, N. Vertolli, R. Wallner, P. Wahlstroem, P. Wilson, S. Zampieri SERENA: particle instrument suite for Sun-Mercury interaction insights on-board BepiColombo. Space Sci. Rev. 217, 300 (2021). https://doi.org/10.1007/ s11214-021-00809-8

P.N. Peplowski et al., Geochemical terranes of Mercury's northern hemisphere as revealed by MESSENGER neutron measurements. Icarus 253, 346-363 (2015). https://doi.org/10.1016/j.icarus.2015.02.002

P.N. Peplowski, R.L. Klima, D.J. Lawrence, C.M. Ernst, B.W. Denevi, E.A. Frank, J.O. Goldsten, S.L. Murchie, L.R. Nittler, S.C. Solomon, Remote sensing evidence for an ancient carbon-bearing crust on Mercury. Nat. Geosci. 9, 273-276 (2016)

E. Quémerais, J.-Y. Chaufray, D. Koutroumpa, F. Leblanc, A. Reberac, B. Lustrement, C. Montaron, J.-F. Mariscal, N. Rouanet, I. Yoshikawa, G. Murakami, K. Yoshioka, O. Korablev, D. Belyaev, M.G. Pelizzo, A.J. Corso, P. Zuppella, PHEBUS on Bepi-Colombo: post-launch update and instrument performance. Space Sci. Rev. 216(4), 1-23 (2020). https://doi.org/10.1007/s11214-020-00695-6

G. Racca, Mercury orbiter mission with solar electric propulsion, ESA/PF/1440.97/GR (1997)

J.M. Raines, G.A. DiBraccio, T.A. Cassidy, D.C. Delcourt, M. Fujimoto, X. Jia, V. Mangano, A. Milillo, M. Sarantos, J.A. Slavin, P. Wurz, Plasma sources in planetary magnetospheres: Mercury. Space Sci. Rev. 192, 91-144 (2015)

D.A. Rothery, M. Massironi, G. Alemanno, O. Barraud, S. Besse, N. Bott, R. Brunetto, E. Bunce, P. Byrne, F. Capaccioni, M.T. Capria, C. Carli, B. Charlier, T. Cornet, G. Cremonese, M. D’Amore, M.C. De Sanctis, A. Doressoundiram, L. Ferranti, G. Filacchione, V. Galluzzi, L. Giacomini, M. Grande, L.G. Guzzetta, J. Helbert, D. Heyner, H. Hiesinger, R. Hyodo, T. Kohout, A. Lucchetti, C. Malliband, P. Mancinelli, J. Martikainen, A. Martindale, A. Maturilli, A. Milillo, A. Morlok, K. Muinonen, O. Namur, L.R. Nittler, J.S. Oliveira, P. Palumbo, M. Pajola, D. Pegg, A. Penttilä, R. Politi, C. Re, C. Stangarone, A. Stojic, T. Väisänen, I. Varatharajan, I. Weber, J. Wright, P. Wurz, F. Zambon, Rationale for BepiColombo studies of Mercury's surface and composition. Space Sci. Rev. 216(4), 1-46 (2020). https://doi.org/10.1007/ s11214-020-00694-7

Y. Saito, J.A. Sauvaud, M. Hirahara, S. Barabash, D. Delcourt, T. Takashima, K. Asamura (the BepiColombo MMO/MPPE Team), Scientific objectives and instrumentation of Mercury Plasma Particle Experiment (MPPE) onboard MMO. Space Sci. Rev. 217(5) (2021). https://doi.org/10.1007/s11214-021-00839-2

F. Santoli, E. Fiorenza, C. Lefevre, D.M. Lucchesi, M. Lucente, C. Magnafico, A. Morbidini, R. Peron, V. Iafolla, ISA, a high sensitivity accelerometer in the interplanetary space. Updates after the near-Earth commissioning phase of Italian Spring Accelerometer/ISA. Space Sci. Rev. 216, 145 (2020). https://doi. org/10.1007/s11214-020-00768-6

D.L. Shirley, The Mariner 10 mission to Venus and Mercury. Acta Astronaut. 53(4-10), 375-385 (2003). https://doi.org/10.1016/S0094-5765(03)00155-3

J.A. Slavin, R.E. Holzer, Solar wind flow about the terrestrial planets, 1. Modeling bow shock position and shape. J. Geophys. Res. 86(A13), 11401-11418 (1981). https://doi.org/10.1029/JA086iA13p11401

J.A. Slavin, G.A. Di Braccio, D.J. Gershman, S.M. Imber, G.K. Poh, J.M. Raines, T.H. Zurbuchen, X. Jia, D.N. Baker, K.-H. Glassmeier, S.A. Livi, S.A. Boardsen, T.A. Cassidy, M. Sarantos, T. Sundberg, A. Masters, C.L. Johnson, R.M. Winslow, B.J. Anderson, H. Korth, R.L. McNutt, S.C. Solomon, MESSENGER observations of Mercury's dayside magnetosphere under extreme solar wind conditions. J. Geophys. Res. Space Phys. 119, 8087-8116 (2014)

J.A. Slavin, D.N. Baker, D.J. Gershman, G.C. Ho, S.M. Imber, S.M. Krimigis, T. Sundberg, Mercury's dynamic magnetosphere, in Mercury the View After MESSENGER, ed. by S.C. Solomon, L.R. Nittler, B.J. Anderson (Cambridge University Press, Cambridge, 2018). Chap. 17

R. Soja, M. Sommer, R. Srama, P. Strub, E. Grün, J. Rodmann, J. Vaubaillon, A. Hornig, L. Bausch, The Interplanetary Meteoroid Environment for eXploration, in Asteroids, Comets, Meteors 2014. Proceedings of the Conference, Helsinki, Finland, 30 June - 4 July, 2014 (2014)

S.C. Solomon, B.J. Anderson, The MESSENGER mission: science and implementation overview, in Mercury-The View After MESSENGER, ed. by S.C. Solomon, L.R. Nittler, B.J. Anderson (Cambridge University Press, Cambridge, 2018), pp. 1-29 
S.C. Solomon, R.L. McNutt, R.E. Gold, M.H. Acuna, D.N. Baker, W.V. Boynton, C.R. Chapman, A.F. Cheng, G. Gloeckler, J.W. Head, S.M. Krimigis, W.E. McClintock, S.L. Murchie, S.J. Peale, R.J. Phillips, M.S. Robinson, J.A. Slavin, D.E. Smith, R.G. Strom, J.I. Trombka, M.T. Zuber, The MESSENGER mission to Mercury: scientific objectives and implementation. Planet. Space Sci. 49, 1445-1465 (2001)

S.C. Solomon, R.L. McNutt Jr., R.E. Gold, D.L. Domingue, MESSENGER mission overview. Space Sci. Rev. 131, 3-39 (2007). https://doi.org/10.1007/s11214-007-9247-6

S.C. Solomon, L.R. Nittler, B.J. Anderson (eds.), Mercury-The View After MESSENGER (Cambridge University Press, Cambridge, 2018). https://doi.org/10.1017/9781316650684 581 p.

R.G. Strom, A.L. Sprague, Exploring Mercury: The Iron Planet (Springer, New York, 2003). ISBN 1852337311

O. Sutherland, D. Stramaccioni, J. Benkhoff, N. Wallace, A. Rocchi, R. Jehn, BepiColombo: ESA's Interplanetary Electric Propulsion mission to Mercury, in The 36th International Electric Propulsion Conference, University of Vienna, Austria, September 15-20 (2019)

N. Thomas, H. Hussmann, T. Spohn, L.M. Lara, U. Christensen, M. Affolter, T. Bandy, T. Beck, S. Chakraborty, U. Geissbuehler, M. Gerber, K. Ghose, J. Gouman, S. HosseiniArani, K. Kuske, A. Peteut, D. Piazza, M. Rieder, A. Servonet, C. Althaus, T. Behnke, K. Gwinner, C. Hüttig, R. Kallenbach, A. Lichopoj, K. Lingenauber, H.-G. Lötzke, F. Lüdicke, H. Michaelis, J. Oberst, R. Schrödter, A. Stark, G. Steinbrügge, S. del Togno, K. Wickhusen, J.M. Castro, M. Herranz, J. Rodrigo, H. Perplies, T. Weigel, S. Schulze-Walewski, S. Blum, A. Casciello, E. Rugi-Grond, W. Coppoolse, M. Rech, K. Weidlich, T. Leikert, R. Henkelmann, B. Trefzger, B. Metz, The BepiColombo Laser Altimeter. Space Sci. Rev. 217, 25 (2021). https://doi.org/10.1007/s11214-021-00794-y

S. Tuttle, G. Cavallo, Thermal design of the Mercury transfer module, in 39th International Conference on Environmental Systems, Savannah, Georgia (2009)

R. van Grieken, A. Markowicz, Handbook of X-Ray Spectrometry: Methods and Techniques (Dekker, New York, 1993)

K.E. Vander Kaaden et al., Geochemistry, mineralogy, and petrology of boninitic and komatiitic rocks on the Mercurian surface: insights into the Mercurian mantle. Icarus 285, 155-168 (2017). https://doi.org/10. 1016/j.icarus.2016.11.041

G. Voisin, I. Cognard, P.C.C. Freire, N. Wex, L. Guillemot, G. Desvignes, M. Kramer, G. Theureau, An improved test of the strong equivalence principle with the pulsar in a triple star system. Astron. Astrophys. 638, A24 (2020). https://doi.org/10.1051/0004-6361/202038104

W.R. Ward, G. Colombo, F.A. Franklin, Secular resonance, solar spin down, and the orbit of Mercury. Icarus 28, 441-452 (1976). https://doi.org/10.1016/0019-1035(76)90117-2

S.Z. Weider et al., Evidence for geochemical terranes on Mercury: global mapping of major elements with MESSENGER's X-Ray Spectrometer. Earth Planet. Sci. Lett. 416, 109-120 (2015). https://doi.org/10. 1016/j.eps1.2015.01.023

S. Yagitani, M. Ozaki, F. Sahraoui, L. Mirioni, M. Mansour, G. Chanteurb, C. Coillot, S. Ruocco, V. Leray, M. Hikishima, D. Alison, O. Le Contel, H. Kojima, Y. Kasahara, Y. Kasaba, T. Sasaki, T. Yumoto, Y. Takeuchi, Measurements of magnetic field fluctuations for Plasma Wave Investigation by the search coil magnetometers (SCM) onboard Bepicolombo Mio (Mercury Magnetospheric Orbiter). Space Sci. Rev. 216(7), 1-26 (2020). https://doi.org/10.1007/s11214-020-00734-2

H. Yamakawa, J. Kawagushi, K. Uesugi, H. Matsuo, Frequent access to Mercury in the early 21st century: multiple Mercury flyby mission via electric propulsion. Acta Astronaut. 39, 133-142 (1996). https:// doi.org/10.1016/S0094-5765(96)00130-0

H. Yamakawa, H. Saito, J. Kawagushi, Y. Kobayashi, H. Hayakawa, T. Mukai, Preliminary ISAS Mercury orbiter mission design. Acta Astronaut. 45, 187-195 (1999). https://doi.org/10.1016/S0094-5765(99) 00136-8

H. Yamakawa, H. Ogawa, Y. Sone, H. Hayakawa, Y. Kasaba, T. Takashima, T. Mukai, BepiColombo Mercury magnetospheric orbiter design, IAC-05-A3.2.B.06, in 56th International Astronautical Congress (IAC), Fukuoka, October 2005 (2005a)

H. Yamakawa, H. Ogawa, Y. Sone, H. Hayakawa, Y. Kasaba, T. Takashima, T. Mukai, BepiColombo Mercury magnetospheric orbiter, in IAA International Conference on Low-Cost Planetary Missions (ICLCPM) Kyoto, 11-13 October (2005b)

C.W. Yen, in Proc. American Aeronautical Society/American Institute of Aeronautics and Astronautics (AAS/AIAA) Astrodynamics Specialist Conference, San Diego, CA (1985). Paper AIAA No. 85-345

C.W. Yen, J. Astronaut. Sci. 37, 417-432 (1989)

I. Yoshikawa et al. (2010). https://doi.org/10.1016/j.pss.2008.07.008

Publisher's Note Springer Nature remains neutral with regard to jurisdictional claims in published maps and institutional affiliations. 


\section{Authors and Affiliations}

J. Benkhoff ${ }^{1}$ (D) - G. Murakami ${ }^{2}$. W. Baumjohann ${ }^{3} \cdot$ S. Besse ${ }^{4} \cdot$ E. Bunce $^{5} \cdot$ M. Casale $^{4}$. G. Cremosese ${ }^{6} \cdot$ K.-H. Glassmeier ${ }^{7} \cdot$ H. Hayakawa ${ }^{8} \cdot$ D. Heyner ${ }^{7} \cdot$ H. Hiesinger ${ }^{9}$.

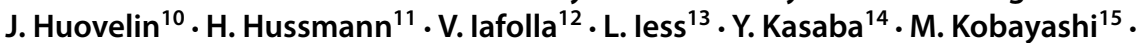
A. Milillo ${ }^{12} \cdot$ I.G. Mitrofanov ${ }^{16} \cdot$ E. Montagnon ${ }^{17} \cdot$ M. Novara ${ }^{1} \cdot$ S. Orsini ${ }^{12}$. E. Quemerais ${ }^{18} \cdot$ U. Reininghaus ${ }^{1} \cdot$ Y. Saito ${ }^{8} \cdot$ F. Santoli ${ }^{12}$ - D. Stramaccioni ${ }^{1}$. O. Sutherland ${ }^{1} \cdot$ N. Thomas $^{19} \cdot$ I. Yoshikawa ${ }^{20} \cdot$ J. Zender ${ }^{1}$

J. Benkhoff Johannes.Benkhoff@esa.int

G. Murakami go.murakami@jaxa.jp

1 ESA/ESTEC, Keplerlaan 1, 2200 AG Noordwijk, The Netherlands

2 JAXA, 3-1-1 Yoshinodai, 299-8510 Sagamihara, Kanagawa, Japan

3 Space Research Institute, Austrian Academy of Sciences, Graz, Austria

4 ESAC, P.O. Box 78, 28691 Vilanueva de la Canada, Spain

5 School of Physics and Astronomy, University of Leicester, University Road, Leicester, LE17RH, UK

6 INAF-Osservatorio Astronomico di Padova, Padova, Italy

7 Institut für Geophysik und Extrateresstrische Physik, Technische Universität Braunschweig, Braunschweig, Germany

8 Institute of Space and Astronautical Science, Japan Aerospace Exploration Agency, Sagamihara, Kanagawa 252-5210, Japan

9 Institut für Planetologie, Westfälische Wilhelms-Universität, Wilhelm-Klemm-Str. 10, 48149 Münster, Germany

10 Department of Physics, University of Helsinki, Helsinki, Finland

11 Institute of Planetary Research, German Aerospace Center, Rutherfordstr. 2, 12489 Berlin, Germany

12 INAF-IAPS (Institute for Astrophysics and Planetology from Space), via del Fosso del Cavaliere 100, 00133, Rome, Italy

13 Dipartimento di ingegneria meccanica e aerospaziale, Universitá La Sapienza, Roma, Italy

14 Planetary Plasma and Atmospheric Research Center, Graduate School of Science, Tohoku University, Sendai, Miyagi 980-8578, Japan

15 Chiba Institute of Technology, Planetary Exploration Research Center, 2-17-1, Tsudanuma, Narashino, Chiba 275-0016, Japan

16 Institute for Space Research, Moscow, Russian Federation

17 European Space Agency, European Space Operations Center, Darmstadt, Germany

18 LATMOS, Universite Versailles Saint-Quentin, Guyancourt, France

19 Physikalisches Institut, University of Bern, Sidlerstr. 5, CH-3012 Bern, Switzerland

20 Department of Complexity Science and Engineering, University of Tokyo, Kashiwa, Chiba 277-13 8561 , Japan 